Aus der Abteilung Ethik und Geschichte der Medizin

(Prof. Dr. med. C. Wiesemann)

im Zentrum Psychosoziale Medizin

der Medizinischen Fakultät der Universität Göttingen

\title{
Beratung zur Patientenverfügung - eine ärztliche Aufgabe?
}

\author{
INAUGURAL-DISSERTATION \\ zur Erlangung des Doktorgrades \\ der Medizinischen Fakultät \\ der Georg-August-Universität zu Göttingen
}

vorgelegt von Julia Windhorst

aus Bielefeld

Göttingen 2012 
Dekan: Prof. Dr. rer. nat. H. K. Kroemer

I. Berichterstatter: PD Dr. phil. A. Simon

II. Berichterstatter/in:

III. Berichterstatter/in:

Tag der mündlichen Prüfung: 


\section{Inhaltsverzeichnis}

EINLEITUNG

2 PATIENTENVERFÜGUNG UND DIE ROLLE DER ÄRZTLICHEN BERATUNG .................................................. 3

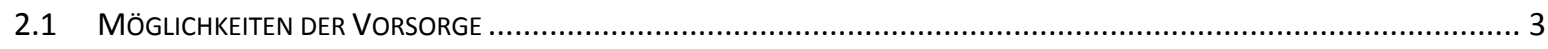

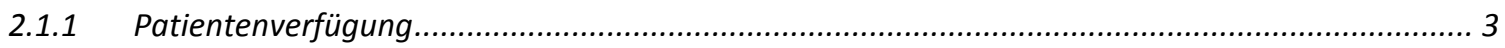

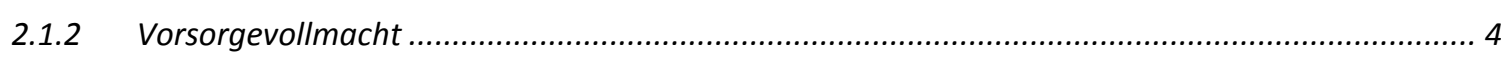

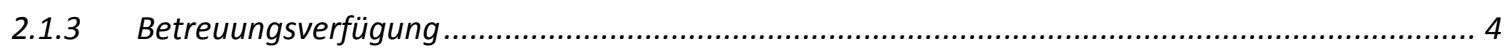

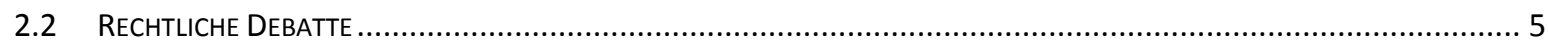

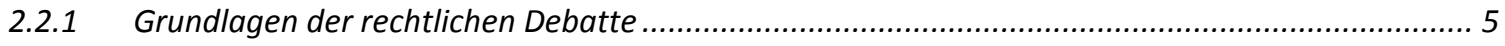

2.2.2 Debatte im Vorfeld des neuen Gesetzes............................................................................ 5

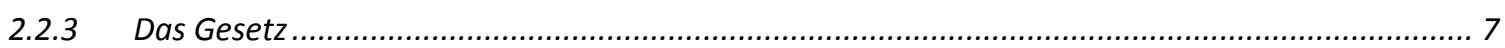

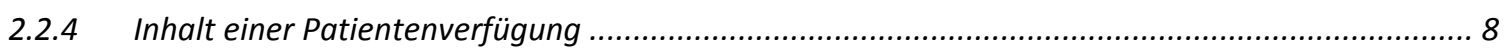

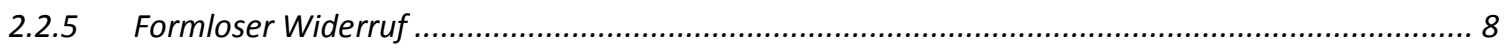

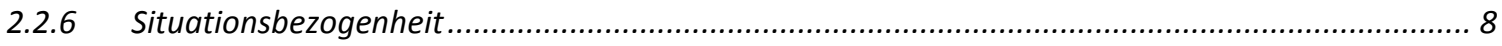

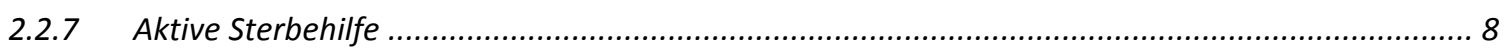

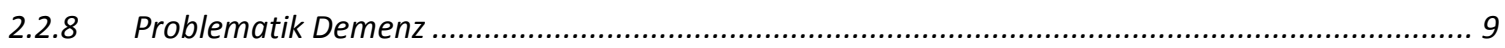

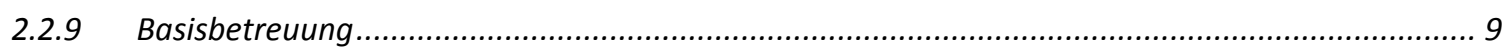

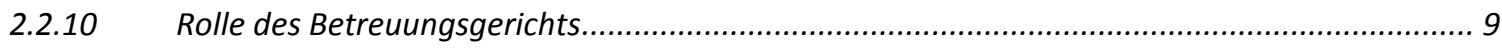

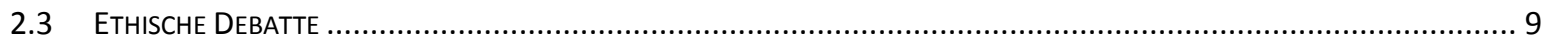

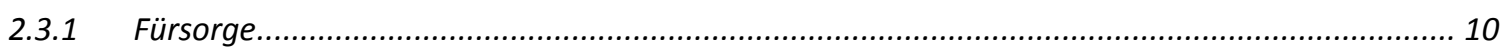

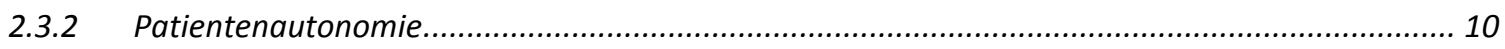

2.3.3 Ethische Probleme bei der Abfassung und Umsetzung von Patientenverfügungen ...................... 11

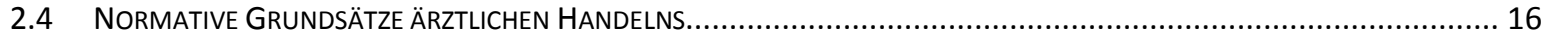

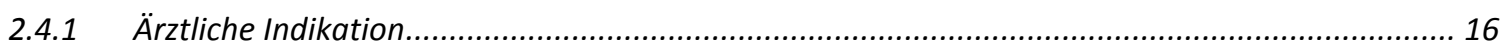

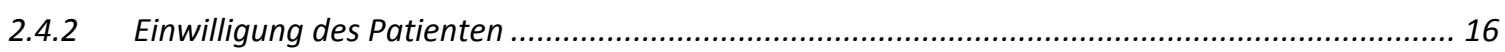

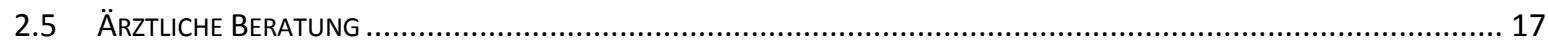

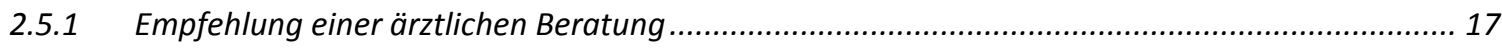

2.5.2 Problematik bei Erstellung einer Patientenverfügung....................................................... 18

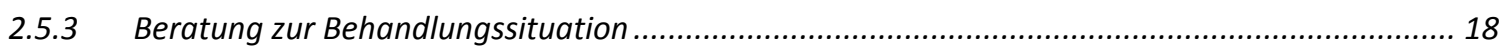

2.5.4 Beratung zu Schaden und Nutzen ärztlicher Eingriffe ......................................................... 18

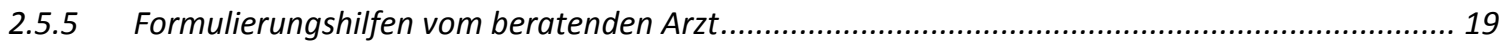

2.5.6 Vorgezogener Dialog zum Meinungsbildungsprozess .......................................................... 19

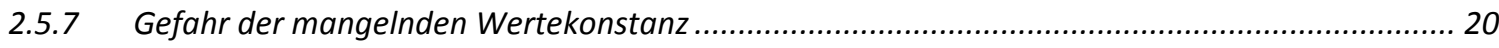

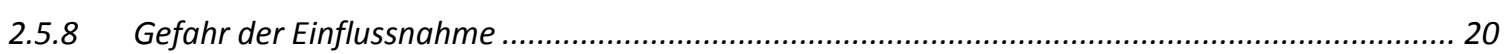

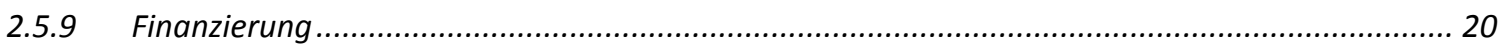




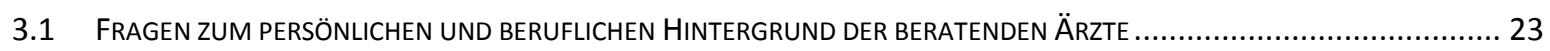

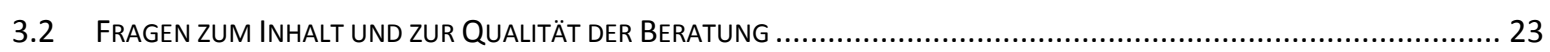

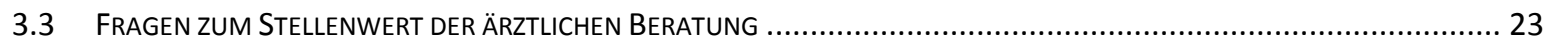

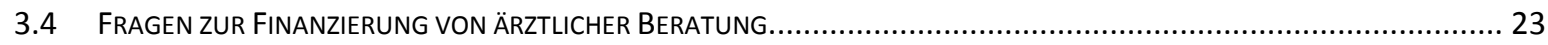

4.1 Die Befragten. 24

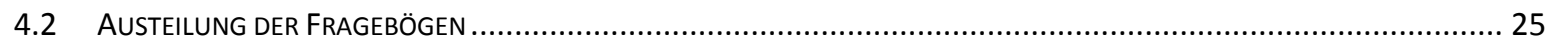

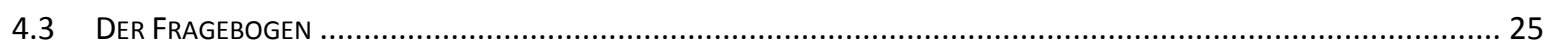

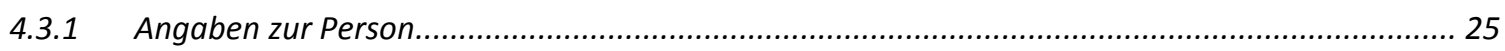

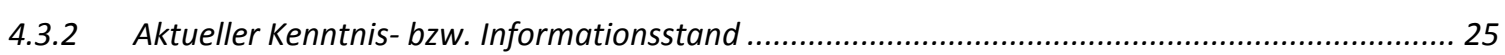

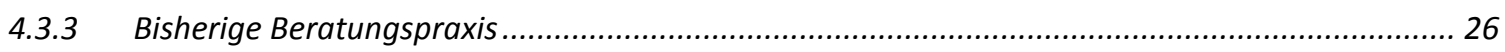

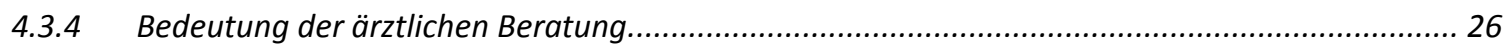

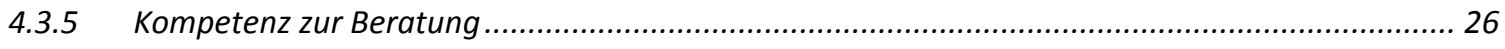

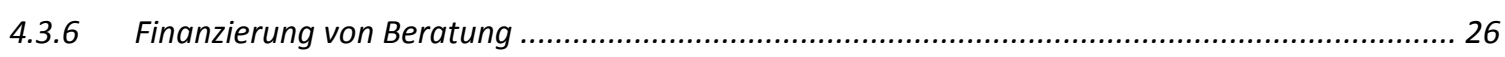

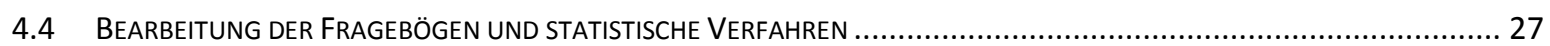

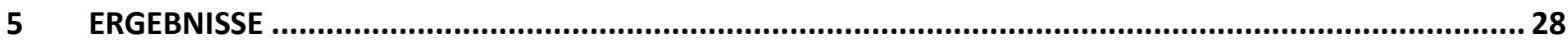

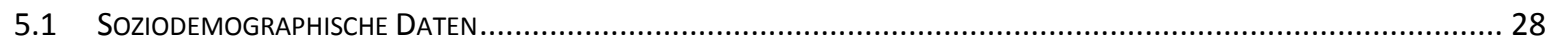

5.2 AKTUELLER INFORMATIONSSTAND SOWIE ALLGEMEINE EINSCHÄTZUNG DER BEDEUTUNG VON PATIENTENVERFÜGUNGEN

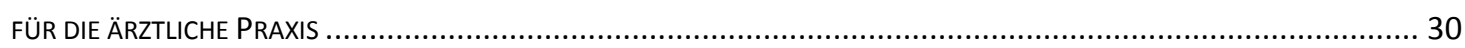

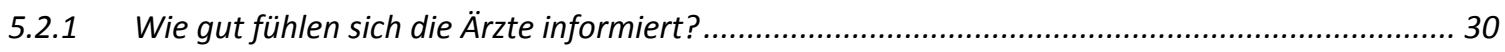

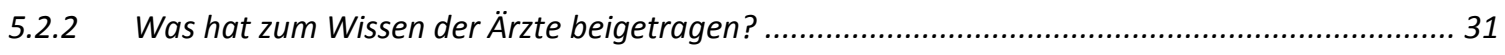

5.2.3 Vereinfacht eine Patientenverfügung die Entscheidungsfindung?......................................... 32

5.2.4 Ist das neue Gesetz eine Hilfe im Umgang mit Patientenverfügungen? ................................... 33

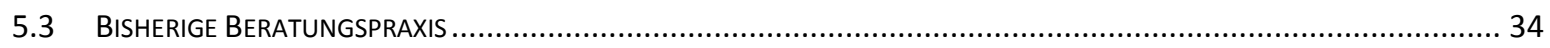

5.3.1 Wie häufig werden Ärzte auf das Thema Patientenverfügung angesprochen?.......................... 34

5.3.2 Hat das neue Gesetz zu mehr Anfragen geführt?......................................................... 35

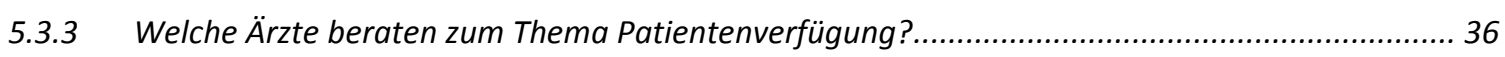

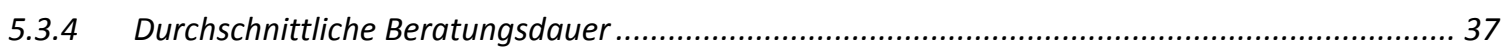

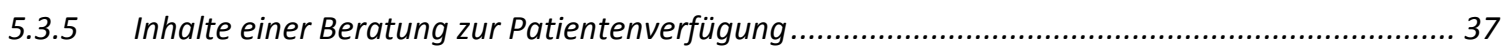

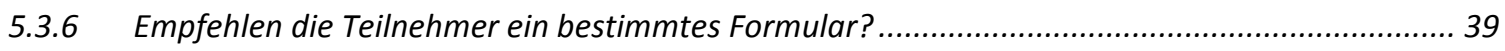

5.3.7 Sprechen Ärzte Patienten auf die Möglichkeit einer Patientenverfügung an?........................... 40

5.3.8 Empfehlen die teilnehmenden Ärzte ihren Patienten die Erstellung einer Patientenverfügung?.. 41

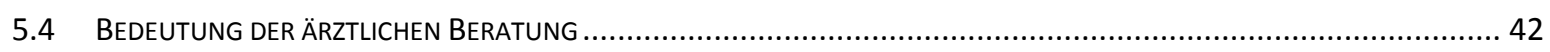

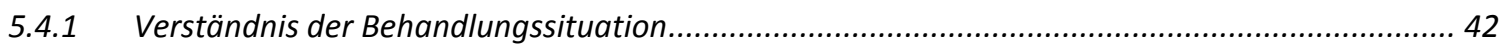

5.4.2 Einschätzung von Schaden und Nutzen ärztlicher Maßnahmen ............................................ 43

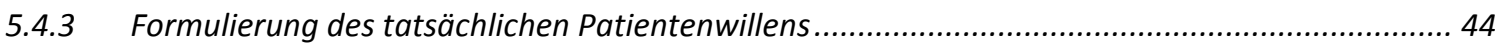


5.4.4 Hilfestellung für den später behandelnden Arzt............................................................... 45

5.4.5 Verbindliche ärztliche Beratung zur Erstellung einer Patientenverfügung............................... 46

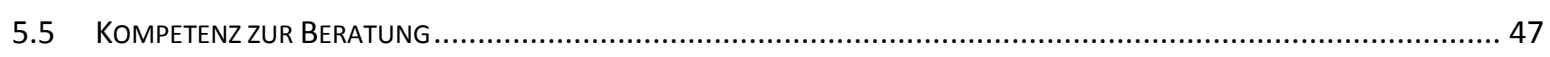

5.5.1 Selbsteinschätzung der Beratungskompetenz............................................................. 47

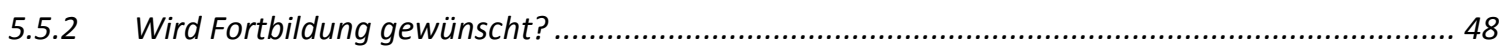

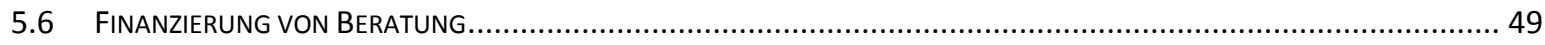

5.6.1 Wurde Beratung zur Patientenverfügung schon abgerechnet? ................................................49

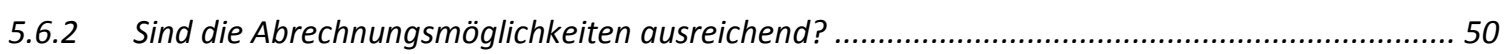

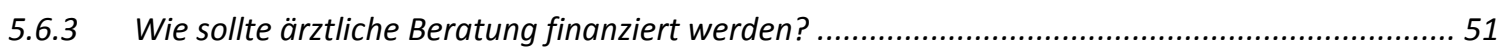

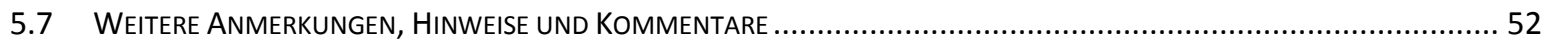

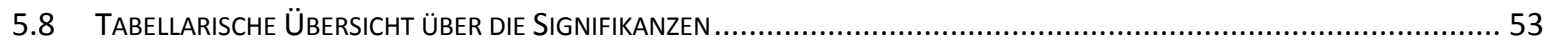

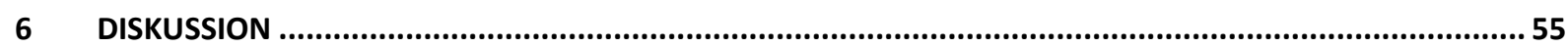

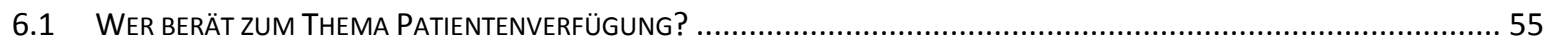

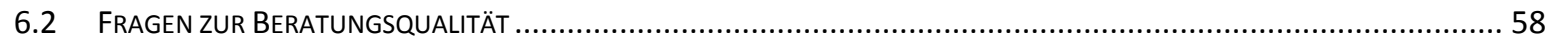

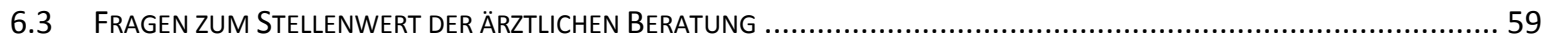

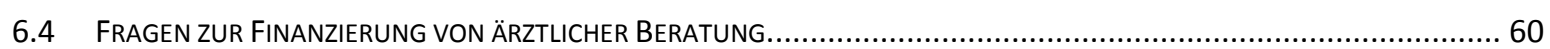

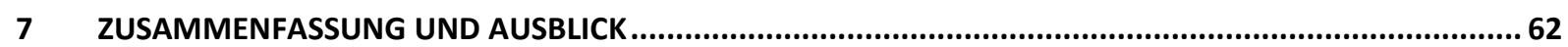

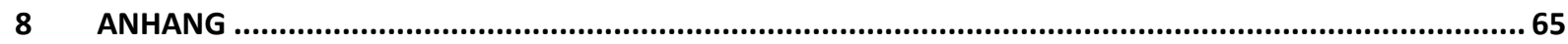

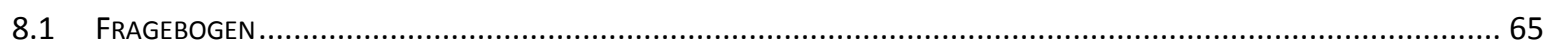

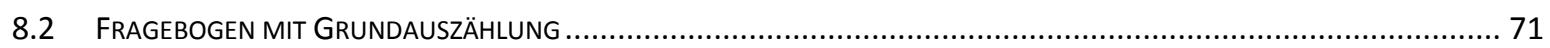

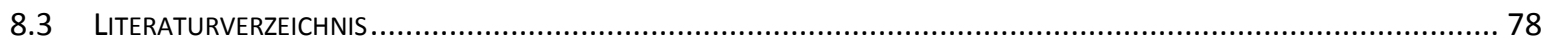




\section{Verzeichnis der Abbildungen und Tabellen}

Abbildung 1: Stellungnahme zu der Aussage: „Ich bin über das Thema PV gut informiert." 30

Abbildung 2: Angaben dazu, welche Medien zum Wissenstand beigetragen haben...... 31

Abbildung 3: Stellungnahme zu der Aussage: „Eine PV vereinfacht die Entscheidungsfindung bei nicht mehr einwilligungsfähigen Patienten.".

Abbildung 4: Stellungnahme zu der Aussage: "Das neue Gesetz vom 1. September 2009 ist eine Hilfe im Umgang 33

Abbildung 5: Wie oft werden die befragten Ärzte auf das Thema PV angesprochen? ..... 34

Abbildung 6: Werden die befragten Ärzte seit Erlass der neuen Regelung häufiger angesprochen? ................. 35

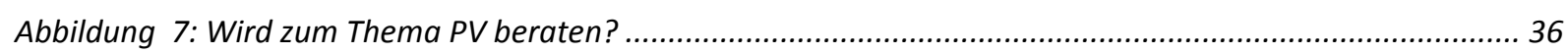

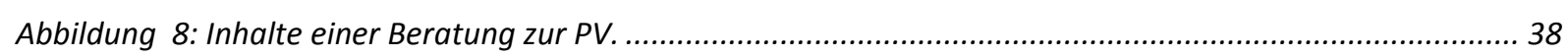

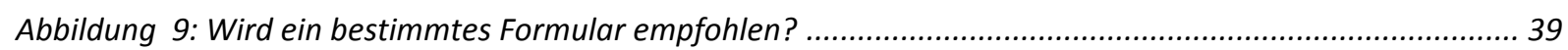

Abbildung 10: Wen sprechen die befragten Ärzte auf die Möglichkeit einer PV an?...................................... 40

Abbildung 11: Wem empfehlen die befragten Ärzte die Erstellung einer PV? ............................................... 41

Abbildung 12: Stellungnahme zu der Aussage: „Ohne ärztliche Beratung kann der Patient die möglichen Behandlungssituationen, auf die er in seiner PV Bezug nimmt, nicht angemessen verstehen. ".... 42

Abbildung 13: Stellungnahme zu der Aussage: "Ohne ärztliche Beratung kann der Patient den möglichen Nutzen und Schaden der ärztlichen Maßnahmen, zu denen er sich in einer PV äußert, nicht angemessen einschätzen."

Abbildung 14: Stellungnahme zu der Aussage: "Ohne ärztliche Beratung besteht die Gefahr, dass der Wortlaut der PV nicht mit dem tatsächlichen Willen des Patienten übereinstimmt."

Abbildung 15: Stellungnahme zu der Aussage: „Ohne ärztliche Beratung ist die PV keine Hilfestellung für den Arzt, der später mit dieser konfrontiert wird." ....

Abbildung 16: Stellungnahme der befragten Ärzte, ob ärztliche Beratung zur PV verbindlich sein sollte. 46

Abbildung 17: Stellungnahme zu der Aussage, ob sich die befragten Ärzte ausreichend kompetent fühlen, Patienten angemessen zur PV zu beraten. 47

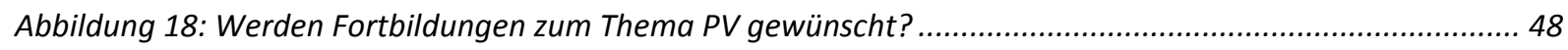

Abbildung 19: Wie Beratungsgespräche bisher abgerechnet wurden.

Abbildung 20: Stellungnahme zu der Aussage: „Die bestehenden Möglichkeiten zur Abrechnung von Beratungsgesprächen sind ausreichend." 50

Abbildung 21: Was sollte ärztliche Beratung zur PV sein?. 51

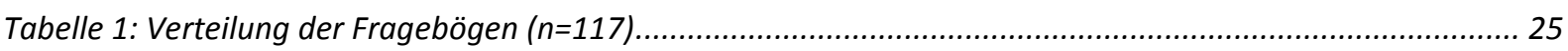

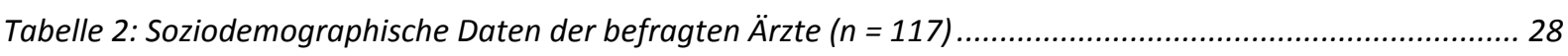

Tabelle 3: Signifikanzen in Bezug auf die Tatsache, ob die befragten Ärzte zum Thema PV beraten oder nicht. . 53 


\section{Abkürzungsverzeichnis}

AG Patientenautonomie
BÄK
BGB
BGH
3. BtÄndG
BV
DFG
Enquete-Kommission
GG
GKV-WSG

PV

SGB

StGB

VV

\author{
Arbeitsgruppe „Patientenautonomie am Lebensende“ \\ Bundesärztekammer \\ Bürgerlicher Gesetzbuch \\ Bundesgerichtshof \\ Drittes Gesetz zur Änderung des Betreuungsrechts \\ Betreuungsverfügung \\ Deutsche Forschungsgemeinschaft \\ Enquete-Kommission Ethik und Recht der modernen Medizin \\ Grundgesetz \\ Gesetz zur Stärkung des Wettbewerbs in der gesetzlichen Krankenversiche- \\ rung \\ Patientenverfügung \\ Sozialgesetzbuch \\ Strafgesetzbuch \\ Vorsorgevollmacht
}




\section{$1 \quad$ Einleitung}

Am 1. September 2009 trat das Dritte Gesetz zur Änderung des Betreuungsrechts in Kraft (BtÄndG 2009). Mit diesem Gesetz wurde die PV in ihrer Bedeutung gestärkt, die nun in ihrer Einhaltung rechtlich bindend ist.

Eine PV ist eine schriftliche Willensbekundung eines volljährigen und einwilligungsfähigen Menschen zu nicht unmittelbar bevorstehenden ärztlichen Maßnahmen in Behandlungssituationen, in denen er nicht mehr entscheidungsfähig ist. Die Verfügung ist unabhängig von Art und Stadium der Erkrankung gültig und kann jederzeit formlos widerrufen werden.

Der Gesetzesänderung ging eine lange öffentliche und politische Debatte voraus, in der viele Teilaspekte des Instruments PV diskutiert wurden. Einer dieser Aspekte war die Rolle der ärztlichen Beratung bei Erstellung einer PV. Die Enquete-Kommission Ethik und Recht der modernen Medizin sprach sich 2004 dafür aus, eine solche Beratung in einem künftigen Gesetz als verbindlich festzuschreiben (Enquete-Kommission 2004). In dem Gesetzesentwurf des Abgeordneten Bosbach war eine ärztliche Beratung als Voraussetzung für die Verbindlichkeit von PVen in nicht sterbensnahen Situationen vorgesehen (Bosbach et al. 2008). In den Entwürfen der Abgeordneten Stünker und Zöller fanden sich hingegen lediglich Empfehlungen für eine Beratung wieder (Stünker et al. 2008 und Zöller et al. 2008). Begründet wurde dies damit, dass niemand zur Information gezwungen werden kann, und dass eine nicht gewollte Aufklärung auch wenig wirkungsvoll ist. Der Stünker-Entwurf wurde in überarbeiteter Form am 18. Juni 2009 vom Bundestag beschlossen.

Warum könnte eine ärztliche Beratung zur PV sinnvoll sein? Ein Arzt hat die Möglichkeit, einen medizinischen Laien, der eine PV für sich erstellen will, umfassend über bestimmte Behandlungssituationen aufzuklären, in denen er einwilligungsunfähig sein könnte. Zudem könnte er angebrachte Behandlungsoptionen darlegen und ausführlich Fragen des zu Beratenden beantworten. Des Weiteren kann ein Arzt sein Fachwissen dazu nutzen, dem zu Beratenden Schaden und Nutzen verschiedener ärztlicher Maßnahmen zu erklären. Mögliche Angst vor intensivmedizinischer Behandlung könnte den Patienten so genommen 
werden (BÄK 2010). Sehr wichtig könnte auch die Hilfestellung bei der Formulierung der PV sein. Ein medizinischer Laie hat eventuell Probleme, seine Behandlungswünsche so zu formulieren, dass sie eindeutig sind. Auf diese Weise können Probleme bei der späteren Umsetzung der PV vermieden werden.

Neben der Frage, ob eine Beratung verbindlich sein sollte, gab es im Vorfeld des neuen Gesetzes auch unterschiedliche Vorschläge, wer diese bezahlen soll. Bosbach schlug in seinem Entwurf eine von der Solidargemeinschaft finanzierte ärztliche Beratung vor. Stünker und Zöller hingegen wollten, dass der Ratsuchende selbst das Beratungsgespräch finanziert.

Um herauszufinden, wie sich die Situation in Bezug auf ärztliche Beratung zur PV nach Inkrafttreten des neuen Gesetzes in der Praxis darstellt, wurde im Rahmen dieser Arbeit eine Umfrage durchgeführt. Ziel der Studie war es herauszufinden, in welchem Ausmaß und in welcher Form Ärzte zum Thema PV beraten. Des Weiteren sollte der Stellenwert ärztlicher Beratung zu diesem Thema aus Sicht der Ärzte verdeutlicht und Fragen zur Finanzierung beleuchtet werden.

Kapitel 2 stellt die verschiedenen Möglichkeiten der Vorsorge (PV, VV und BV) vor und erläutert die aktuelle Rechtslage. Ferner wird die Rolle der ärztlichen Beratung vor dem Hintergrund der rechtlichen und ethischen Debatte um die PV beleuchtet. In Kapitel 3 wird die Fragestellung der Studie konkretisiert. Kapitel 4 stellt Material und Methode vor. In Kapitel 5 werden die Ergebnisse der Studie dargestellt und in Kapitel 6 diskutiert. Kapitel 7 fasst die wichtigsten Ergebnisse der Studie zusammen und erläutert deren Bedeutung für die weitere Debatte. 


\section{$2 \quad$ Patientenverfügung und die Rolle der ärztlichen Beratung}

Die Frage nach der Rolle der ärztlichen Beratung zur PV steht im engen Zusammenhang zu der Bedeutung, die der PV als solcher bei medizinischen Entscheidungen im Falle von nicht einwilligungsfähigen Patienten eingeräumt wird. Im Folgenden sollen deshalb zunächst die verschiedenen Instrumente der Vorsorge (PV, VV, BV) sowie die rechtlichen und ethischen Debatten um diese vorgestellt werden, bevor dann auf die Frage der ärztlichen Beratung bei Erstellung einer PV eingegangen wird.

\subsection{Möglichkeiten der Vorsorge}

\subsubsection{Patientenverfügung}

Eine PV ist ein schriftliches Dokument einer einwilligungsfähigen volljährigen Person, in der diese festlegt, ob sie „in bestimmte, zum Zeitpunkt der Festlegung noch nicht unmittelbar bevorstehende Untersuchungen ihres Gesundheitszustands, Heilbehandlungen oder ärztliche Eingriffe einwilligt oder sie untersagt“ (BGB §1901a Abs. 1). Die PV ist unabhängig von Art und Stadium der Erkrankung verbindlich und kann jederzeit formlos, d.h. auch mündlich oder durch eine Geste, widerrufen werden.

Die PV kann um Bitten oder Richtlinien, wie z. B. den Hinweis auf eine gewünschte häusliche Pflege, erweitert werden. Zudem kann auch der Beweggrund, weswegen der Patient die vorliegende Verfügung verfasst hat, hinzugefügt werden. Auch kann es sinnvoll sein, persönliche Wertvorstellungen, die eigene Einstellung zu Leben und Tod sowie die eigenen religiösen Anschauungen zu nennen. Dies erleichtert es im Anwendungsfall, die PV auf die konkrete Situation hin auszulegen. Es handelt sich hierbei um Ergänzungen, die im engen Sinne keine PV darstellen, da sie sich nicht auf ärztliche Maßnahmen beziehen.

Eine sinnvolle Ergänzung zur PV ist die Benennung einer Vertrauensperson im Rahmen einer VV und/oder BV. Nach deutschem Recht haben weder Ehepartnerinnen oder Ehepartner, noch Eltern und ihre erwachsenen Kinder untereinander die Befugnis, ihre Partnerinnen, 
Partner oder nächsten Verwandten in medizinischen Angelegenheiten rechtswirksam zu vertreten. Es muss diesbezüglich vorher eine Vollmacht für diese Person erteilt worden sein. Ist dies nicht geschehen, muss vom Betreuungsgericht ein gesetzlicher Vertreter bestellt werden, der dann befugt ist, in medizinische Behandlungen einzuwilligen oder diese abzulehnen.

\subsubsection{Vorsorgevollmacht}

In einer VV wird eine Vertrauensperson schriftlich benannt, die bei Verlust der Entscheidungsfähigkeit als rechtsverbindlicher Vertreter des Patienten auftreten soll. Dies geschieht bei Vorlage einer zulässigen VV direkt und ohne Einschalten des Betreuungsgerichts (BGB $§ 1896$ Abs. 2).

Durch eine VV wird dem behandelnden Arzt ein Ansprechpartner zu Seite gestellt. Dieser hat zu prüfen, ob der in der PV geäußerte Wille die konkrete Behandlungssituation erfasst und in der akuten Lebenssituation Gültigkeit besitzt. Andernfalls hat er aufgrund konkreter Hinweise den mutmaßlichen Patientenwillen zu ermitteln und gegenüber dem Arzt zur Geltung zu bringen.

Soll der Bevollmächtigte auch in weitgreifende und gefährliche Heilbehandlungen einwilligen bzw. ärztlich indizierte lebensverlängernde Maßnahmen ablehnen, so muss dies ausdrücklich in der VV erwähnt werden (BGB §1904 Abs. 5).

\subsubsection{Betreuungsverfügung}

Die BV muss ebenfalls schriftlich vorliegen und ist dazu da, eine geschäftsfähige und volljährigen Person oder mehrere Personen als gesetzlichen Betreuer vorzuschlagen (BGB $\S 1897$ Abs. 4). Ferner können in einer BV Vorschläge hinsichtlich der Art und Weise der Betreuung gemacht werden. Das Gericht prüft die Vorschläge und hat ihnen zu entsprechen, sofern sie nicht dem Wohl des Patienten zuwiderlaufen. Es würde beispielsweise eingreifen, wenn die vorgeschlagene Betreuungsperson ungeeignet ist.

Die BV kann zusätzlich zur VV erstellt werden, um auch in Situationen, in denen Zweifel an der Zuständigkeit der VV bestehen - etwa dann, wenn die Formulierungen in der VV nicht eindeutig den Aufgabenbereich des Bevollmächtigten beschreiben - die gesetzliche Vertretung durch die benannte Person zu sichern. Die BV kann aber auch anstelle der VV verfasst werden, für den Fall, dass eine stärkere Kontrolle des gesetzlichen Stellvertreters 
durch das Betreuungsgericht erwünscht wird. Da der Betreuer dem Gericht regelmäßig Bericht erstatten muss, steht er unter einer impliziten Kontrolle des Richters.

\subsection{Rechtliche Debatte}

\subsubsection{Grundlagen der rechtlichen Debatte}

Bis zum September 2009 war das Instrument PV gesetzlich nicht geregelt. VV (BGB §§ 1896 Abs. 2 und 1901c) und auch BVen ( $\S 1897$ Abs. 4 BGB) sind hingegen schon länger im Betreuungsrecht verankert.

Erklärtes Ziel der aktuellen Gesetzesänderung war es, das Selbstbestimmungsrecht des Patienten zu stärken. Das Selbstbestimmungsrecht ist im Grundgesetz in den Artikeln 1 und 2 verankert. In Artikel 1 heißt es: „Die Würde des Menschen ist unantastbar. Sie zu achten und zu schützen ist Verpflichtung aller staatlichen Gewalt" (GG Art. 1, Abs. 1). In Zeiten, in denen der medizinische Fortschritt immer weiter voranschreitet und die Intensivmedizin immer mehr Möglichkeiten bietet, Leben zu erhalten, haben viele Menschen das typische Bild der „Apparatemedizin“ vor Augen und sehen darin die „Würde des Menschen“ am Lebensende nicht mehr gegeben. Im Artikel zwei des Grundgesetzes wird betont, dass „jeder das Recht auf die freie Entfaltung seiner Persönlichkeit“ (GG Art. 2 Abs. 1) und „das Recht auf Leben und körperliche Unversehrtheit" hat (GG Art. 2 Abs. 1). Dies legt nahe, dass eine PV eine effektive Möglichkeit darstellt, auch im Falle einer Entscheidungsunfähigkeit seine eigenen moralischen Vorstellungen von einem „Sterben in Würde“ zu verwirklichen.

Auf der anderen Seite steht eine andere Auslegung des Grundgesetzes. Der Staat hat die Aufgabe, das „Recht auf Leben“ zu schützen. Dies bezieht sich vor allem auf den Schutz vor Dritten, jedoch auch darauf, den Menschen vor sich selbst zu schützen (Hufen 2009; Kämpfer 2007; Hillgruber 2006). So ist in Deutschland beispielsweise unter bestimmten Voraussetzungen eine stationäre Zwangsbehandlung erlaubt, wenn der Patient nicht einwilligungsfähig ist und dabei eine erhebliche Selbst- oder Fremdgefährdung besteht.

\subsubsection{Debatte im Vorfeld des neuen Gesetzes}

Mit der Entscheidung des Bundesgerichtshofs zum „Lübecker Fall“ vom 17. März 2003 intensivierte sich die Debatte um eine gesetzliche Regelung zum Thema PV. In seiner Entscheidung setzte das Gericht eine konkrete und situationsbezogene PV dem erklärten Willen des Patienten gleich (BGH 2003). Dies stärkte die Bedeutung der PV erheblich, warf 
aber neue Fragen auf. Diese betrafen vor allem die Reichweite von PVen, sowie die Rolle des Betreuungsgerichts. Da diese nicht durch richterliche Rechtsfortschreibung allein zu beantworten sind, empfahlen die Richter eine gesetzliche Regelung der Thematik. Damit brachte der BGH die Politik in einen Zugzwang. Eine gesetzliche Regelung sollte gefunden werden.

Das Bundesjustizministerium setzte daraufhin die interdisziplinäre Arbeitsgruppe „Patientenautonomie am Lebensende“ (AG Patientenautonomie) ein, die im Juni 2004 ihren Bericht vorlegte. Dieser beinhaltete die Empfehlung, die PV im Betreuungsrecht zu regeln, wobei sich die AG für eine weitreichende Verbindlichkeit konkreter und situationsbezogener PVen aussprach und eine Einschaltung des Betreuungsgerichts nur in Konfliktsituationen vorsah. Erklärtes Ziel war die Stärkung des Selbstbestimmungsrechts des Patienten.

Nur zwei Monate später reagierte die vom Deutschen Bundestag eingesetzte EnqueteKommission „Ethik und Recht der modernen Medizin“ (Enquete-Kommission 2004) mit einem Zwischenbericht. Sie warnte vor einer einseitigen Betonung des Selbstbestimmungsrechts und rückte die Bedeutung der Prinzipien Fürsorge und Lebensschutz in den Vordergrund. Die Mehrheit der Mitglieder empfahlen, die Gültigkeit einer PV in einem künftigen Gesetz „auf Fallkonstellationen zu beschränken, in denen das Grundleiden irreversibel ist und trotz medizinischer Behandlung nach ärztlichen Erkenntnissen zum Tode führen wird“ (Enquete-Kommission 2004). Zudem sollte eine bindende Ablehnung von lebenserhaltenden Maßnahmen im Rahmen von Demenzerkrankungen und Wachkoma nicht möglich sein. Um den nicht mehr einwilligungsfähigen Patienten vor der Ablehnung einer lebenserhaltenden Maßnahme durch den Bevollmächtigten/Betreuer zu schützen, sollte ein beratendes Konsil verpflichtend eingeschaltet werden, das aus dem behandelnden Arzt, dem rechtlichen Vertreter, einem Angehörigen und einem Vertreter des Pflegeteams bestehen sollte. Zusätzlich sollte es außerhalb sterbensnaher Situationen einer Genehmigung des Betreuungsgerichts bedürfen.

Das Bundesjustizministerium präsentierte im November 2004 einen Gesetzentwurf basierend auf den Vorschlägen der AG Patientenautonomie, welcher aber wieder zurückgezogen wurde, da Kritiker meinten das neue Gesetz zur PV dürfe nicht von der Regierung kommen, sondern sollte vom Bundestag selbst eingebracht werden. 
Im Bundestag diskutiert und zur Abstimmung gebracht wurden die Entwürfe von drei fraktionsübergreifenden AGn, die sich um die Abgeordneten Bosbach, Zöller und Stünker zusammenfanden. Alle drei Entwürfe beinhalteten, dass die in einer PV festgelegten Wünsche und Entscheidungen zu beachten und vom Bevollmächtigten bzw. Betreuer umzusetzen sind. Sie sollten jederzeit formlos widerrufen werden können. Alle drei Entwürfe brachten zum Ausdruck, dass aktive Sterbehilfe in einer PV nicht gefordert werden kann.

Während die Entwürfe von Zöller und Stünker die Verbindlichkeit von PVen unabhängig von Art und Stadium der vorliegenden Erkrankung vorsahen, knüpfte Bosbach die Verbindlichkeit der PV in Situationen, in denen keine unheilbare, tödlich Erkrankung vorliegt, an eine ärztliche und rechtliche Beratung, eine notarielle Beurkundung sowie eine Aktualisierung alle fünf Jahre, ferner sollte ein Abbruch lebenserhaltender Maßnahmen nur auf der Grundlage eines vorangegangenen Konsils und einer richterlichen Genehmigung möglich sein (Bosbach et al. 2008).

Bei Bosbach war die ärztliche Beratung eine Voraussetzung für die Verbindlichkeit von PVen in nicht sterbensnahmen Situationen. Die Abgeordneten Stünker und Zöller lehnten eine solche „indirekte Reichweitenbeschränkung“ ab. Sie sprachen sich aber auch unabhängig davon gegen eine verpflichtende Beratung mit der Begründung aus, dass niemand zur Beratung gezwungen werden kann. Außerdem stuften sie eine verpflichtende Beratung als „kaum wirkungsvoll“ ein. Trotzdem empfahlen sie eine ärztliche Beratung zur PV, genauso wie eine regelmäßige Aktualisierung. In der Begründung zu dem Entwurf von Stünker heißt es: „Verzichtet der Verfasser auf eine fachkundige Beratung, trägt er das Risiko einer fehlenden Bindungswirkung seiner PV aufgrund nicht hinreichend konkreter Formulierungen“ (Stünker 2008, S.14). Der unterschiedlichen Bedeutung der PV entsprechend, gaben die Entwürfe unterschiedliche Antworten auf die Frage, wer die Kosten für eine solche Beratung übernehmen soll: Bosbachs Entwurf sah die Vergütung von ärztlicher Beratung zur PV als eine von der Solidargemeinschaft zu tragende Leistung an. Zöller und Stünker sahen darin eine vom Patienten selbst zu finanzierende Leistung.

\subsubsection{Das Gesetz}

Der Entwurf von Stünker wurde am 18. Juni 2009 im Bundestag mit einer Mehrheit von 317 Stimmen, bei 233 Gegenstimmen und fünf Enthaltungen angenommen. Die Entwürfe von Bosbach und Zöller sowie der Vorschlag einer Gruppierung um den Abgeordneten Hüppe, 
die eine gesetzliche Regelung der PV ablehnte, fanden keine Mehrheiten. So trat das Dritte Gesetz zur Änderung des Betreuungsrechts am 1. September 2009 in Kraft. Die Bestimmungen des neuen Gesetzes sollen im Folgenden kurz vorgestellt werden.

\subsubsection{Inhalt einer Patientenverfügung}

In einer PV legt der Verfasser fest, welche diagnostischen oder therapeutischen Maßnahmen er für den Fall der eigenen Einwilligungsunfähigkeit in bestimmten noch nicht unmittelbar bevorstehenden Krankheitssituationen wünscht bzw. nicht mehr wünscht. Die Festlegungen sind unabhängig von Art und Stadium der Erkrankung verbindlich (BGB §1901a). Die PV ist also nicht auf Aussagen zu lebenserhaltenden Maßnahmen am Lebensende beschränkt.

\subsubsection{Formloser Widerruf}

Die PV ist für Betreuer und Arzt bindend. Der Patient selbst kann diese jedoch jederzeit formlos - d.h. auch mündlich oder durch eine Geste - widerrufen (BGB §1901a Abs. 1). Ein Ablaufdatum für PVen gibt es nicht, d.h. auch ältere PVen behalten ihre Gültigkeit, sofern es keinen konkreten Hinweis gibt, dass der Patient selbst diese nicht mehr als verbindlich ansieht.

\subsubsection{Situationsbezogenheit}

Wichtig bei der Ermittlung des Patientenwillens ist die Situationsbezogenheit. Alles was in einer PV schriftlich festgelegt wurde, muss bei der Ausführung auf die aktuelle Lebens- und Behandlungssituation bezogen und daraufhin beurteilt werden. Die in der PV beschriebenen Behandlungssituationen müssen mit der aktuell zu beurteilenden Situation übereinstimmen, ebenso konkret genannte ärztliche Maßnahmen.

Die Ermittlung der aktuellen Lebenssituation ist schwierig. Es muss genau geprüft werden, ob sich der Patientenwille in der nun eingetretenen Situation geändert hat. Falls der Arzt Anzeichen dafür festgestellt hat, dass sich der Patientenwille geändert hat und nicht mehr dem in der PV niedergeschriebenen Willen entspricht, muss er dies begründen.

\subsubsection{Aktive Sterbehilfe}

Auch in einer PV kann der Patient nur fordern, was rechtlich erlaubt ist. Der Wunsch nach einer strafrechtlich verbotenen aktiven Sterbehilfe ist daher nicht zulässig. 


\subsubsection{Problematik Demenz}

Eine besondere Problematik betrifft den Umgang mit Demenzerkrankungen. Sind natürliche Willensäußerungen (z.B. vom Pflegepersonal wahrgenommene Anzeichen von Lebensfreude) ein Widerruf der PV? Grundsätzlich gilt, dass eine aktuelle Willensbekundung Vorrang hat, doch ist für einen Widerruf die Einwilligungsfähigkeit des Patienten erforderlich? Die Antwort auf diese Frage ist umstritten und schwierig: Auf der einen Seite droht die Gefahr, dass nicht mehr einwilligungsfähige Patienten zum Sklaven ihrer früheren Willenserklärung werden, auf der anderen Seite ist zu befürchten, dass der natürliche Wille dazu missbraucht wird, frühere Willenserklärungen auszuhebeln (Taupitz 2008; Verrel 2006).

\subsubsection{Basisbetreuung}

Maßnahmen der Basisbetreuung, wie Körperpflege, das Lindern von Schmerzen, Übelkeit und Atemnot oder das Stillen von Hunger und Durst auf natürlichem Weg, sollten von einer PV nicht erfasst, sondern vorausgesetzt werden (Stünker 2008). Bei Nichtbeachtung würden sonst auch die Rechte Dritter missachtet, wie z. B. durch Infektionsgefahr aufgrund mangelnder Körperpflege.

\subsubsection{Rolle des Betreuungsgerichts}

Die Einschaltung des Betreuungsgerichts ist in $\S 1904$ BGB geregelt. Danach ist eine Genehmigung des Betreuungsgerichts bei stellvertretenden Entscheidungen, die die Einwilligung in gefährliche Heileingriffe oder die Nichteinwilligung in lebenserhaltende Maßnahmen zum Inhalt haben, nur dann erforderlich, wenn zwischen Arzt und Betreuer keine Einigkeit besteht, dass die Entscheidung des Betreuers dem Willen des Patienten entspricht. Weiterhin genehmigungsbedürftig sind stellvertretende Entscheidungen über freiheitsentziehende bzw. -beschränkende Maßnahmen (§ 1906 BGB).

\subsection{Ethische Debatte}

In der ethischen Debatte um die PV steht die Frage nach einer angemessenen Gewichtung der Prinzipien Autonomie und Fürsorge im Mittelpunkt. Im Folgenden werden zunächst die Begriffe Fürsorge und Autonomie vorgestellt. Danach wird auf ethische Probleme eingegangen, die sich bei der Abfassung bzw. Umsetzung einer PV ergeben können. 


\subsubsection{Fürsorge}

Schon historisch begründete sich das ärztliche und pflegerische Berufsethos verstärkt auf die Fürsorge und den Lebensschutz dem Patienten gegenüber. Dies ist fest verankert in den traditionellen Kodizes wie dem Hippokratischen Eid (Lichtenthaeler 1984), dem Eid von Florence Nightingale (Bostridge 2009) und der Genfer Deklaration des Weltärztebundes von 1948 (Genfer Deklaration 1948, Z. 4). In Letzterem heißt es: „Die Gesundheit meines Patienten soll oberstes Gebot meines Handelns sein."

Grundsätzlich drückt Fürsorge das für jemanden Sorgen aus. Als Synonym könnte man auch Betreuung, Hilfe, Obhut oder sogar Bemutterung verwenden (Duden 2000). Gerade letzteres drückt eine stark schützende Komponente des Wortes aus, die letztlich wiederum auf den Lebensschutz bezogen werden kann.

Nun geht es in PVen häufig um Entscheidungen am Lebensende. Wenn man Fürsorge mit Lebensschutz, also der Erhaltung des Lebens, in Verbindung bringt, so wird in vielen PVen ja das Gegenteil gefordert: die Unterlassung lebenserhaltender Maßnahmen. Dies könnte vom behandelnden Arzt ein Handeln gegen seine eigenen moralischen und ethischen Einstellungen fordern.

In den letzten Jahrzehnten hat sich aber der Stellenwert dieser Werte in der Gesellschaft zunehmend verändert. Während bis Ende des 20. Jahrhunderts ein eher paternalistisches Verhältnis zwischen Arzt und Patient herrschte, so liegt die Betonung nun vermehrt auf der Patientenautonomie. Schon vor dem Dritten Gesetz zur Änderung des Betreuungsrechts stärkten verschiedene richterliche Beschlüsse das Selbstbestimmungsrecht des Patienten. Das am 1. September 2009 in Kraft getretene Gesetz regelt die Verbindlichkeit der PV und ist Ausdruck und Folge dieser Entwicklung.

\subsubsection{Patientenautonomie}

Die Patientenautonomie wurde in der zweiten Hälfte des 20. Jahrhunderts zu einem zentralen Prinzip in der Medizinethik. Faden und Beauchamp benennen drei notwendige Voraussetzungen für eine autonome Entscheidung: Diese muss von einer Person, die (1) versteht, worum es geht, (2) bewusst und (3) ohne steuernde Einflussnahme Dritter getroffen werden (Faden und Beauchamp 1986).

Die erste Voraussetzung ist das Verständnis. Der Patient muss die Behandlungssituation sowie die verschiedenen Behandlungsoptionen verstehen. Aus einem bestehenden 
Wissensdefizit könnte ein erhebliches Autonomiedefizit resultieren. Dies verdeutlicht die Bedeutung der ärztlichen Aufklärung für eine autonome Patientenentscheidung.

Die zweite Voraussetzung ist die Zielgerichtetheit der Entscheidung. Ein Patient muss sich bewusst sein, in welchem Rahmen er eine Entscheidung trifft und welche Folgen, Chancen und Risiken seine Entscheidung mit sich bringen kann.

Die Dritte Voraussetzung ist, dass die Entscheidung ohne steuernde Einflussnahme Dritter gefällt wird. Entscheidend hierbei ist das Adjektiv „steuernd“. Denn kaum eine Entscheidung findet ohne Einflussnahme Dritter statt. Auch in der Beratung seitens der Ärzte oder in der Begleitung von Freunden und Verwandten wird meist eine direkte oder indirekte, sowie verbale oder nonverbale Favorisierung einer Option mitklingen. Trotzdem sollte der Patient abschließend unabhängig entscheiden können.

\subsubsection{Ethische Probleme bei der Abfassung und Umsetzung von Patientenverfügungen}

\subsubsection{Motivation zur Erstellung einer Patientenverfügung}

Viele Menschen, die eine PV erstellen wollen, haben Angst, am Ende ihres Lebens der „Apparatemedizin“ ausgeliefert zu sein. Sie kommen über Krankheitsfälle im Familien- oder Bekanntenkreis auf das Thema PV und haben den Wunsch, eben nicht an „Schläuchen und Geräten hängend“ zu sterben. Eine von der DFG geförderte Studie, die nach der Motivation zur Erstellung einer PV fragte, kam zu dem Ergebnis, dass 51 \% der Befragten durch den Tod eines Verwandten oder Freundes auf die Idee kamen, eine PV zu erstellen. Nur 14 \% nannten ihre eigene Erkrankung als Motivationsgrund (Jaspers et al. 2010), d. h., dass einige Menschen beim Verfassen einer PV keine konkrete Erkrankung vorzuweisen haben. Es gibt noch keine Anhaltspunkte, in welche konkreten Situationen sie in einem möglichen Krankheitsfall mit Verlust ihrer Entscheidungskompetenz geraten könnten. Sie haben noch keine Vorstellung, wie sie sich in so einer Situation fühlen und welche subjektiven Erfahrungen sie mir einer Krankheit machen könnten. Ihr Wissen über intensivmedizinische Maßnahmen ist eventuell begrenzt auf Erfahrungen, die sie bei Besuchen von Familienangehörigen oder Bekannten gemacht haben. Die moderne Medizin hat die Grenzen von Leben und Tod verschieben können, häufig zum Wohl und Genesung von schwerstkranken Menschen. Doch bei allen Erfolgen, die die Intensivmedizin 
hervorzubringen mag, sind ihr doch Grenzen gesetzt. Ab einem gewissen Punkt besteht keine Aussicht mehr auf Heilung und eventuell nicht einmal mehr eine Aussicht auf eine Besserung des Gesundheitszustands. In dieser Situation müssen sich Patienten, deren Angehörigen und die behandelnden Ärzte die Frage stellen, wann medizinische Maßnahmen ein Leiden sinnlos in die Länge ziehen und ob die akute Behandlungssituation den moralischen Vorstellungen von "würdevollem Leben“ des Patienten entspricht. Diese Behandlungssituationen vorherzusehen, den Schaden und Nutzen der intensivmedizinischen Maßnahmen zu erörtern und alles konkret in Worte zu fassen ist nun für eine gültige PV wichtig. Die Frage ist, ob ein normaler Bürger ohne medizinische Ausbildung und ohne professionelle Beratung dazu im Stande ist.

\subsubsection{Behandlungssituationen}

Für einen medizinischen Laien ist es schwierig, die möglichen Behandlungssituationen, in denen seine PV zum Einsatz kommen könnte, zu antizipieren und angemessen einzuschätzen. Auch für den Arzt selbst ist es oft nicht möglich, die konkreten Umstände einer künftigen Behandlungssituation vorherzusagen. Ohne diese Informationen ist es jedoch nur schwer möglich, konkrete Aussagen zu Art und Umfang der in diesen Situationen gewünschten Behandlung zu treffen. Ein Patient, der bereits an einer Krankheit leidet, kann die möglichen Behandlungssituationen eventuell besser beurteilen. Dennoch bleiben meist so viele verschiedene Szenarien, die eintreten könnten, dass eine detaillierte antizipierte Beschreibung unmöglich erscheint.

\subsubsection{Einschätzung von Schaden und Nutzen verschiedener Therapieoptionen}

Vor allem der medizinische Laie hat kaum eine Möglichkeit, sich bei der Erstellung einer PV über den Schaden und Nutzen der verschiedenen, eventuell intensivmedizinischen, Therapiemethoden detailliert zu informieren. Häufig führen Vorurteile gegenüber der „Apparatemedizin“ zu Fehleinschätzungen aus Sicht des Patienten. Dies könnte dazu führen, dass Patienten bestimmte Behandlungen ablehnen oder aber ihnen zustimmen, ohne genau zu wissen, welche Vor- und Nachteile diese Optionen mit sich bringen. In diesem Rahmen könnten die Therapiemöglichkeiten ebenso unter- wie überschätzt werden. 


\subsubsection{Formulierungen}

In einer PV sollten die Situationen so konkret wie möglich formuliert sein, wobei eine detaillierte Beschreibung in Unkenntnis der späteren Behandlungssituation kaum durchführbar ist. Hinterlässt ein Patient Anweisungen für sehr konkrete Situationen, besteht die Gefahr, dass er eine später eintretende Situation nicht bedacht hat oder diese nicht genau genug auf eine beschriebene Situation passt und somit die gewünschte Therapie nicht umgehend ausgeführt werden kann. Bleibt die Beschreibung des Behandlungsprocedere jedoch zu vage, so kann auch dies ebenso die Durchführung einer gewünschten Therapieoption erschweren oder verhindern. Dies ist eine Problematik, die kaum optimal zu lösen ist. Auch wird dieses Dilemma nicht unbedingt dadurch erleichtert, dass viele Patienten vorgefasste Formulare benutzen. Da einige auf möglichst viele Menschen zutreffen sollen, sind sie sehr allgemein gehalten und geben nicht den individuellen Willen des Patienten wieder. Formulare, die mit Textbausteinen, bzw. ankreuzbaren Behandlungssituationen- und wünschen arbeiten, bringen nur bedingt Verbesserung.

Auch wenn der zukünftige Patient eine genaue Vorstellung davon hat, wie das gewünschte Behandlungsprocedere ablaufen soll, so können Probleme bei der Formulierung einer PV bestehen. Ein medizinischer Laie kann seine Wünsche nur bedingt verbal konkretisieren, was zu Missverständnissen führen könnte.

\subsubsection{Fehlende Entwicklungsmöglichkeiten im Meinungsbildungsprozess}

Im ärztlichen Alltag werden Entscheidungen nach Möglichkeit im Dialog mit dem Patienten getroffen. Wird eine aus ärztlicher Sicht klar indizierte Therapiemöglichkeit vom Patienten abgelehnt, so sollte nach den aktuellen Grundsätzen der BÄK zur ärztlichen Sterbebegleitung ein erneuter Dialog mit dem Patienten stattfinden, um ihm die Möglichkeit zu geben, seine Entscheidung zu überdenken (BÄK 2011). Diese Möglichkeit besteht im Rahmen der Erstellung einer PV natürlich nicht.

Das Erstellen einer PV kann dafür vielmehr eine Möglichkeit darstellen, sich intensiv mit dem Thema „Entscheidungen am Lebensende“ zu befassen und sich eventuell auch mit einer Erkrankung auseinanderzusetzen. In diesem Rahmen kann sich ein Mensch mit eigenen Vorstellungen von Würde und Wünschen für den Fall einer Einwilligungsunfähigkeit auseinandersetzen, Informationen zu seiner Krankheit und eventuell eintretenden Behandlungssituationen sammeln und damit eine sehr differenzierte und durchdachte PV 
erstellen. Eine qualitative Befragung von Patienten kam 2001 so zu dem Ergebnis, dass das Erstellen einer PV durchaus als Teil der Krankheitsbewältigung gesehen wird (Schäfer 2001).

\subsubsection{Gefahr der mangelnden Wertekonstanz}

Dem Patienten sollte bewusst sein, welche Reichweite sein schriftlich abgefasster Wille für ihn haben kann. Er muss in Betracht ziehen, dass sich sein Wille durchaus, vor allem in Zeiten einer schweren Krankheit, mit der Zeit wandeln kann. Die Erfahrungen zeigen, dass in Krankheitssituationen bestimmte Mobilitätseinschränkungen und Einschränkungen durch Schmerzen, sowie das Angewiesensein auf Hilfe und/oder auf medizinische Technik ganz anders empfunden werden als in gesunden Tagen. Studien zeigen, dass gerade Patienten mit einer infausten Prognose signifikant häufiger eingreifende Therapiemaßnahmen wünschen (Sahm 2006). Dies spricht für einen Perspektivenwechsel am Lebensende, der kaum von einem gesunden Patienten beim Verfassen einer PV berücksichtigt werden kann.

Das Fällen von Entscheidungen über wichtige medizinische Maßnahmen ist häufig ein prozeduraler Prozess. Viele unterschiedliche Faktoren im Erleben einer Krankheit fließen in einen Meinungsbildungsprozess mit ein, die sich fortwährend ändern und vorab gar nicht nachempfunden werden können. Wie sich ein Patient in einer bestimmten Krankheitssituation fühlt und inwiefern sich neue Behandlungsergebnisse und Hoffnungen auf einen positiven Ausgang einer Behandlung auf sein Empfinden und seinen Zustand auswirken und damit zu einem Perspektivenwechsel gegenüber einem vorher geäußerten Meinungsbild führen könnten, ist beim Verfassen einer PV kaum vorauszusehen.

Obwohl Werte, Einstellungen und Entscheidungen meist keine konstanten Größen darstellen und deswegen durchaus die Gefahr besteht, dass ein Perspektivenwechsel die Gültigkeit einer PV infrage stellt, so ist doch anzunehmen, dass Patienten mit PV eine höhere Wertekonstanz zeigen, da sie sich eingehend mit dem Thema beschäftigt haben.

Besonders schwerwiegend sind Entscheidungen am Lebensende im Zusammenhang mit dementiellen Erkrankungen und Wachkomapatienten. Diese zeigen eine schwerwiegende Diskontinuität der Persönlichkeit. Wird ein Patient nun nach einer PV behandelt, die er im gesunden Zustand erstellt hat, bezeichnet die Enquete-Kommission dies als eine „Versklavung“ der aktuellen Person, durch die frühere Person (Enquete-Kommission 2004). Würde aber eine dritte Person entscheiden, so wäre dies genauso eine „Versklavung“, 
nämlich durch diese dritte Person, die nie eine Beeinflussung durch eigene Interessen, bewusst oder unbewusst, ausschließen kann.

\subsubsection{Gefahr der Einflussnahme}

Es ist sicherlich keine unbegründete Sorge, dass manche PVen aus dem Grund, für andere keine Last mehr darzustellen, erstellt werden. Wir leben in einer Gesellschaft, in der Menschen immer älter werden und zunehmend dementielle Erkrankungen das Leben im Alter erschweren und die Angst vor dem Älterwerden steigern.

Die Finanzierbarkeit unseres Gesundheitssystems wird immer mehr in der Öffentlichkeit zur Sprache gebracht und könnte in Zeiten der Ressourcenknappheit einen erheblichen Druck auf alte und kranke Menschen ausüben, durch eine PV ihr Leben selbstbestimmt beenden zu lassen. Eine Umfrage der Deutschen Hospizstiftung aus dem Jahr 2005 kam zu dem Ergebnis, dass 23 \% der über 60-Jährigen eine PV erstellt hatten. Sie begründeten ihre Entscheidung für eine PV unter anderem damit, „niemandem zur Last fallen“ zu wollen (45 \%) und „anderen keine Kosten verursachen zu wollen“ (26 \%) (Deutsche Hospizstiftung 2005).

Diese Begründung könnte durchaus ein Hinweis auf die Gefahr sein, dass eine PV nicht ganz ohne steuernde Einflussnahme Dritter, sei sie nun gewollt oder nicht gewollt, verfasst wird.

\subsubsection{Sterbehilfe}

Im Rahmen der Diskussionen um PVen kommt auch die Sorge der Kritiker hinzu, dass durch PVen mehr und mehr die Grenzen zwischen erlaubter passiver bzw. indirekter und verbotener - und nur in den Niederlanden, Belgien und Luxemburg erlaubter - aktiver Sterbehilfe verschwimmen könnten. Eine Studie von Wissenschaftlern aus Göttingen und Jena zeigte große Unsicherheiten bei Ärzten und auch bei Betreuungsrichtern, ob bestimmte ärztliche Handlungen, wie z. B. das Beenden der künstlichen Ernährung oder Beatmung, der aktiven oder passiven Sterbehilfe zuzuordnen sind (Simon et al. 2004 und Oorschot et al. 2006). Nun kommt hinzu, dass indirekte und passive Sterbehilfe auch aufgrund von PVen durchgeführt werden dürfen. Da in einigen Formblättern explizit auf die deutsche Rechtslage hingewiesen wird, kann eine Patientenverfügung nun eher dazu beitragen, dass Unsicherheiten in diesem Zusammenhang seltener auftreten (Bayerisches Staatsministerium der Justiz 2011). Die ethische Auseinandersetzung mit dem Thema PV macht deutlich, dass das Erstellen einer PV keine einfach Lösung für Entscheidungen am Lebensende bei 
fehlender Entscheidungskompetenz darstellt. Viele Faktoren zeigen, wie schwierig die Erstellung einer PV sein kann und welche Folgen dies mit sich ziehen kann. Es ist wichtig, Entscheidungen von Patienten zu respektieren und umzusetzen, was letztlich durch das 3. BtÄndG auch gesetzlich verpflichtend geregelt ist (3. BtÄndG 2009). Doch auch der Aspekt der Fürsorge bleibt, der es dem Arzt zur Aufgabe macht, PVen kritisch zu prüfen und in Interaktion mit dem Bevollmächtigten den mutmaßlichen Willen des Patienten zu ermitteln, um im Konflikt zwischen Selbstbestimmung und Fürsorge einen Weg zu gehen, der möglichst beiden Seiten gerecht wird.

\subsection{Normative Grundsätze ärztlichen Handelns}

\subsection{1 Ärztliche Indikation}

Grundlegende Voraussetzung für die Durchführung einer diagnostischen oder therapeutischen Maßnahme ist die ärztliche Indikation und die Einwilligung des angemessen aufgeklärten Patienten.

Die Indikationsstellung ist Aufgabe des Arztes. Er entscheidet, basierend auf seinem medizinischen Wissen und seiner ärztlichen Erfahrung, welche Maßnahmen in einer bestimmten Behandlungssituation angezeigt sind. Diese bietet er dem Patienten an. Er weist auf die Vor- und Nachteile der verschiedenen Behandlungsoptionen hin. Ist eine Maßnahme ohne medizinischen Nutzen oder bringt sie dem Patienten nach Einschätzung des Arztes mehr Schaden als Nutzen, so wird sie dem Patienten gar nicht erst angeboten und auch dann nicht durchgeführt, wenn der Patient diese wünscht.

\subsubsection{Einwilligung des Patienten}

Über die Durchführung der vom Arzt angebotenen Maßnahmen entscheidet - von Notfallsituationen abgesehen - der Patient durch seine Einwilligung. Wird eine Maßnahme gegen den Willen des Patienten durchgeführt, so stellt dies nach deutschem Recht eine vorsätzliche Körperverletzung dar (St GB § 223). Dies gilt auch für lebenserhaltende oder lebensverlängernde Maßnahmen. Die Einwilligung des Patienten ist sehr wichtig und soll auf einem angemessenen Entscheidungsfindungsprozess basieren. Der behandelnde Arzt hat die Aufgabe, diesen Prozess angemessen zu begleiten. Hierzu gehört die ärztliche Aufklärung zu den bevorstehenden Eingriffen oder der anstehenden Therapie. Der Arzt sollte sorgfältig die Vor- und Nachteile der möglichen Behandlungsmaßnahmen abwägen und die Risiken 
gewissenhaft erwähnen und erklären. Nur ein gut aufgeklärter Patient kann eine autonome Entscheidung treffen, die alle Faktoren berücksichtigt und individuell begründet ist (Faden und Beauchamp 1986).

Der Arzt hat im Verlauf der Entscheidungsfindung keinen bestimmenden Charakter, wohl aber kann er durch fachkräftige Beratung dem Patienten helfen, die für den Patienten richtige Entscheidung zu treffen. Er kann in diesem Rahmen seiner ärztlichen Fürsorge Ausdruck verleihen.

\section{5 Ärztliche Beratung}

\subsubsection{Empfehlung einer ärztlichen Beratung}

Aus dem vorangegangen Abschnitt geht hervor, wie wichtig eine ärztliche Aufklärung für eine autonome Patientenentscheidung ist. Dies trifft auch auf die Erstellung einer PV zu. Denn ohne ärztliche Aufklärung wird es für einen medizinischen Laien schwierig sein, konkrete Vorgaben für bestimmte Behandlungssituationen zu geben. Auf die Bedeutung der ärztlichen Aufklärung wurde auch in den drei Gesetzesentwürfen für das 3. BtÄndG hingewiesen. Im Bosbach-Entwurf war die ärztliche Beratung sogar eine der Voraussetzungen für die Verbindlichkeit von PVen in nicht sterbensnahen Situationen (Bosbach et al. 2008).

Letztlich hat sich der Gesetzgeber gegen eine verpflichtende ärztliche Beratung zu PV entschieden. In der Gesetzesbegründung heißt es: “Es kann durchaus in der Absicht des Verfassers liegen, lediglich allgemeine Richtlinien für künftige medizinische Behandlungen festzuhalten, die dann als Indiz für den mutmaßlichen Patientenwillen vom Vertreter zu berücksichtigen sind“ (Stünker 2008 S. 14). Dies ist natürlich auch ohne ärztliche Beratung möglich. Auch verfassen Menschen, die im medizinischen Bereich tätig sind, eine PV. Diese brauchen keine ärztliche Beratung, da sie aus Erfahrungen und erlerntem Wissen alle Voraussetzungen zum Erstellen einer PV erfüllen. Auch denen, die bewusst auf die ärztliche Beratung zur PV verzichten, muss es möglich sein, eine PV zu erstellen.

Zugleich wird in der Gesetzesbegründung aber auch darauf hingewiesen, dass die Erstellung einer PV ohne ärztliche Beratung das Risiko birgt, dass der Wille des Patienten nicht ausreichend gut formuliert sein könnte. Diese Auffassung vertritt auch die BÄK. In ihrer Empfehlung zum Umgang mit VV und PV in der ärztlichen Praxis heißt es: „Patienten sind 
danach häufig erst durch eine fachkundige Beratung in der Lage, Formulierungen zu finden, die geeignet sind, ihre persönlichen Vorstellungen hinreichend nachvollziehbar und umsetzbar niederzulegen“ (BÄK 2007, vgl. Stünker et al. 2008 S. 14). Ein weiterer Punkt, den die BÄK für wichtig erachtet, ist die Einschätzung der Behandlungssituationen. Durch Patientenberatung durch den Arzt kann dieser „Informationen für das Abwägen der Entscheidung beitragen“ (BÄK 2010).

Die wichtigsten Gründe zur ärztlichen Beratung zur PV sind also die Aufklärung des Patienten über mögliche Behandlungssituationen und die Unterstützung bei der Formulierung der Behandlungswünsche.

\subsubsection{Problematik bei Erstellung einer Patientenverfügung}

Bei der Erstellung einer PV muss der Verfasser die Behandlungssituationen, in denen die PV zum Tragen kommen könnte, antizipieren, den möglichen Nutzen und Schaden verschiedener ärztlicher Maßnahmen in diesen Situationen verstehen und seine Behandlungswünsche unmissverständlich formulieren. Für alle drei Schritte kann eine ärztliche Beratung von Vorteil sein.

\subsubsection{Beratung zur Behandlungssituation}

Ein Arzt hat die Möglichkeit, umfassend über verschiedene Behandlungssituationen zu informieren. Er hat das medizinische Fachwissen und die Erfahrung, über gewisse Szenarien, in die ein Patient kommen kann und in denen er einwilligungsunfähig werden könnte, aufzuklären, und die aus seiner Sicht angezeigten Behandlungsoptionen darzulegen. Außerdem hat er die Möglichkeit, auf den zu beratenden Patienten individuell einzugehen und seine eventuell schon bestehenden Krankheiten in diese Szenarien zu integrieren. Je genauer eine mögliche Behandlungssituation eingegrenzt werden kann, desto konkreter können Behandlungsanweisungen formuliert werden.

\subsubsection{Beratung zu Schaden und Nutzen ärztlicher Eingriffe}

Der Arzt kann den Patienten ferner über den möglichen Nutzen und Schaden verschiedener Behandlungsoptionen aufklären. Fehleinschätzungen im Sinne übertriebener Hoffnungen oder unbegründeter Ängste seitens des Patienten z.B. mit Blick auf die Möglichkeiten der Intensivmedizin können dadurch korrigiert werden (BÄK 2010). 


\subsubsection{Formulierungshilfen vom beratenden Arzt}

Ist das Verständnis der möglichen Behandlungssituationen und die Einschätzung über Nutzen und Schaden der ärztlichen Maßnahmen gegeben, so kann der beratende Arzt dem Patienten helfen, seine Behandlungswünsche so zu formulieren, dass sie für die Ärzte, die diese später umsetzen sollen, unmissverständlich sind. Ohne ärztliche Beratung besteht die Gefahr, dass die Formulierungen missverständlich sind oder möglicherweise sogar das Gegenteil von dem zum Ausdruck bringen, was der Patient gemeint hat. Diese Gefahr, die für manche Ärzte auch der Grund für eine skeptische Haltung gegenüber dem Instrument der PV darstellt, kann durch eine ärztliche Aufklärung weitgehend gebannt werden.

\subsubsection{Vorgezogener Dialog zum Meinungsbildungsprozess}

Auch für das Ärzteteam, welches später die Verantwortung für den Patienten hat und den Willen des Patienten am Lebensende möglichst genau umsetzen möchte, kann eine vorangegangene ärztliche Beratung hilfreich sein. Eine gültige PV mit möglichst genauer Beschreibung der Behandlungssituationen und konkreten Angaben zu den vom Patienten gewünschten ärztlichen Maßnahmen vereinfacht die Umsetzung sehr. Hat eine ärztliche Beratung stattgefunden, so sollte dies in der PV vermerkt werden. Das Ärzteteam hat dadurch nicht das Gefühl, dass der Patient vielleicht etwas völlig anderes gemeint hat und kann bei eventuellen Rückfragen den vermerkten Arzt kontaktieren. Dieser hat im Verlaufe des Beratungsgesprächs vielleicht einen genaueren Eindruck von den Wünschen und Vorstellungen des Patienten erlangt und kann dies an die jetzt behandelnden Ärzte weitergeben. Der Dialog zwischen Arzt und Patient kann zwar nicht situationsbezogen stattfinden, wird aber zumindest so gut wie möglich vorgezogen.

Hat ein ausführliches Beratungsgespräch stattgefunden, konnte der beratende Arzt den Patienten bei der Auseinandersetzung mit den Szenarien am Lebensende und dem Umgang mit eventuell bestehenden Vorerkrankungen ein Stück weit begleiten. Der Patient steht mit der Auseinandersetzung und Verarbeitung mit dem Thema nicht alleine da. Auch ist es sinnvoll, zusätzlich einen Bevollmächtigen $\mathrm{zu}$ bestimmten und diesen zum Beratungsgespräch hinzuzubitten. Im Fall der späteren Entscheidungsunfähigkeit des Patienten steht der Bevollmächtigte als Dialogpartner für die behandelnden Ärzte zur Verfügung. 


\subsubsection{Gefahr der mangelnden Wertekonstanz}

Die Beratung durch einen Arzt bei Erstellung einer PV schließt nicht aus, dass der Patient seine Behandlungswünsche ändert. Die ärztliche Beratung kann jedoch helfen, unüberlegte oder vorschnelle Festlegungen zu vermeiden. Außerdem kann sie dem Patienten dabei helfen, sich der Möglichkeit einer Willensänderung bewusst zu werden. Der Hinweis, dass eine PV jederzeit formlos widerrufen werden kann, kann den Patienten dahingehend entlasten, dass er nicht an seinen einmal festgelegten Willen gebunden ist, sondern diesen jederzeit ändern kann.

\subsubsection{Gefahr der Einflussnahme}

Ein zur PV beratender Arzt kann die Gefahr der Einflussnahme durch Faktoren wie „keinem zur Last fallen wollen“ oder „den großen finanziellen Aufwand einer aufwendigen Pflege scheuen" nicht abwenden. Wohl aber kann er versuchen, sich mit dem Patienten zusammen mit diesen Gefühlen zu beschäftigen und damit dem Patienten in dieser akuten Situation zu helfen.

\subsubsection{Finanzierung}

Eine nach wie vor offene Frage ist die Frage nach der Finanzierung der ärztlichen Beratung zur PV. Zwar hat sich der Gesetzgeber mit der Begründung, dass niemand zu einer Beratung gezwungen werden darf, und dass eine solche Pflichtberatung wenig sinnvoll sei, gegen eine Pflichtberatung ausgesprochen. Andererseits wird in der Gesetzesbegründung selbst sowie in diversen Stellungnahmen auf die Bedeutung der ärztlichen Beratung bei der Erstellung einer PV hingewiesen. Der fehlende Pflichtcharakter der PV schließt eine solidarische Finanzierung nicht aus. Im Gegenteil: Wenn durch eine solche die möglichen Probleme bei der Erstellung und Umsetzung von PVen verringert werden können, so könnte es durchaus im Sinne der Solidargemeinschaft sein, durch eine - zumindest teilweise - Übernahme der Beratungskosten, möglichst viele Versicherte zu motivieren, sich vor Erstellung einer PV ärztlich beraten zu lassen.

Zusammenfassend kann man feststellen, dass zur Entstehung einer validen PV, die konkrete Behandlungswünsche in verschiedenen Behandlungssituationen beschreibt, eine ärztliche Beratung sinnvoll ist und von offiziellen Stellen empfohlen wird. Ärztliche Aufklärung stärkt 
Patientenverfügung und die Rolle der ärztlichen Beratung

den Aspekt der Fürsorge dem Patienten gegenüber. Nur der gut informierte Patient kann eine autonome Entscheidung treffen. Beratung als Ausdruck der ärztlichen Fürsorge stärkt also auch die Autonomie des Patienten. 


\section{$3 \quad$ Fragestellung}

Im vorangegangenen Teil wurden die aktuelle Debatte sowie mögliche Probleme im Zusammenhang mit der Erstellung und Umsetzung von PVen dargestellt. Insbesondere im Hinblick auf die Rolle der ärztlichen Beratung besteht noch ein erheblicher Klärungsbedarf. In verschiedene Stellungnahmen von Expertengremien wird eine ärztliche Beratung vor Erstellung einer PV angeraten (AG Patientenautonomie 2004, Enquete-Kommission 2004, BÄK 2007 und 2010). Im aktuellen Gesetz zur PV wird eine ärztliche Beratung zwar nicht als verbindlich vorgeschrieben, in der Gesetzesbegründung jedoch als sinnvoll empfohlen (Stünker et al. 2008).

Genauere Vorgaben zu den Zielen und Inhalten einer ärztlichen Beratung gibt es jedoch kaum. In den Empfehlungen der BÄK und der Zentralen Ethikkommission heißt es nur allgemein: „So kann der Arzt beispielsweise über medizinisch mögliche und indizierte Behandlungsmaßnahmen informieren, auf die mit Prognosen verbundenen Unsicherheiten aufmerksam machen und allgemein über Erfahrungen mit Patienten, die sich in vergleichbaren Situationen befunden haben, berichten. Indem der Arzt den Patienten möglichst umfassend informiert, kann er zugleich Vorsorge gegen aus ärztlicher Sicht nicht gebotene Festlegungen des Patienten treffen, etwa indem er über Missverständnisse - z. B. über die sogenannte Apparatemedizin - aufklärt, Fehleinschätzungen hinsichtlich der Art und statistischen Verteilung von Krankheitsverläufen korrigiert und die Erfahrungen aus dem Umfeld des Patienten, an denen sich dieser orientiert und aus denen er möglicherweise falsche Schlüsse zieht, hinterfragt" (BÄK 2010 S. A 880).

Dies lässt viele Fragen offen: Welche Ärzte sollen zur PV beraten? Was könnten die Inhalte der Beratung sein, und in welcher Form sollte sie erfolgen? Welche Qualifikation muss ein Arzt haben, wenn er zum Thema PV berät? Wie kann er diese Qualifikationen erwerben?

Um herauszufinden, in welchem Ausmaß und in welcher Form ärztliche Beratung zum Thema PV stattfindet, und welche Qualität diese Beratung hat, wurde im Rahmen der vorliegenden Arbeit eine Studie durchgeführt, in der Ärztinnen und Ärzte aus verschiedenen Bereichen zum Thema „Beratung zur PV - eine ärztliche Aufgabe?“ mittels Fragebogen befragt wurden. Dabei interessierten insbesondere folgende Fragen: 


\subsection{Fragen zum persönlichen und beruflichen Hintergrund der beratenden Ärzte}

- Unterscheiden sich die beratenden Ärzte von den nicht beratenden Ärzten hinsichtlich bestimmter soziodemografischer Daten wie Alter, Geschlecht oder Facharztausbildung?

- Wird in niedergelassenen Praxen häufiger beraten als im Krankenhaus?

- Beraten Ärzte, die für sich selbst eine PV erstellt haben, häufiger zum Thema PV als solche, die selbst keine PV haben?

\subsection{Fragen zum Inhalt und zur Qualität der Beratung}

- Was sind die Inhalte des Beratungsgesprächs?

- Wie lange dauert ein Beratungsgespräch?

- Weisen Ärzte auf die Möglichkeit einer PV hin?

- Empfehlen Ärzte die Erstellung einer PV?

- Wie gut fühlen sich die beratenden Ärzte informiert?

- Was hat zum Wissen der Ärzte beigetragen?

- Wünschen sich die beratenden Ärzte Fortbildung zum Thema?

\subsection{Fragen zum Stellenwert der ärztlichen Beratung}

- Wird ärztliche Beratung zum Thema PV als wichtig angesehen?

- Wenn ja, aus welchen Gründen?

- Sollte nach Meinung der Ärzte eine ärztliche Beratung vor Erstellung einer PV verbindlich sein?

- Gibt es Unterschiede in der Einschätzung des Stellenwerts ärztlicher Beratung zwischen beratenden und nicht beratenden Ärzten?

\subsection{Fragen zur Finanzierung von ärztlicher Beratung}

- Wie werden Beratungsgespräche bislang abgerechnet?

- Werden die bestehenden Abrechnungsmöglichkeiten als ausreichend angesehen?

- Soll die Beratung zur PV eine von der Solidargemeinschaft oder vom Patienten selbst zu finanzierende Leistung sein? 


\section{$4 \quad$ Material und Methoden}

Die Umfrage untersucht, wie und in welchem Umfang Ärzte im Krankenhaus und im niedergelassenen Bereich zum Thema PV beraten. Insbesondere geht es um die Frage, inwiefern Ärzte die Beratung zur PV als eine ärztliche Aufgabe betrachten.

\subsection{Die Befragten}

Als Probanden wurden Ärztinnen und Ärzte ausgewählt, die an der Zusatz-Weiterbildung Palliativmedizin teilnahmen. Die Weiterbildung besteht aus einer Kursweiterbildung (früher: Basiskurs) sowie drei darauf aufbauenden Fallseminaren. Im Basiskurs sowie im Fallseminar Modul 2 wird explizit auf ethische und rechtliche Fragen am Lebensende und in diesem Zusammenhang auch auf die Bedeutung sowie auf mögliche Probleme im Umgang mit PVen eingegangen. Im Fallseminar Modul 3 werden Fälle der Teilnehmer besprochen; auch hier werden regelmäßig ethische und rechtliche Fragen im Zusammenhang mit der PV thematisiert.

Es konnte also davon ausgegangen werden, dass ein hoher Anteil der Befragten bereits eigene Erfahrungen im Umgang mit PVen hatte, und einige der Teilnehmer auch Beratungen zur PV durchführten. Letzteres war vor allem deshalb von Bedeutung, da sich ein Teil der Fragen auf den Inhalt und die Qualität der Beratung bezogen. Ein weiterer Grund für die Probandenauswahl bestand in der Tatsache, dass die Weiterbildung von Ärzten unterschiedlicher Fachgebiete sowie aus dem niedergelassenen und stationären Bereich besucht wurde. Da die Fragebögen im Rahmen der Kurse ausgeteilt und ausgefüllt wurden, konnte darüber hinaus eine hohe Rücklaufquote erwartet werden. 


\subsection{Austeilung der Fragebögen}

Die Fragebögen wurden zwischen März und Juni 2010 in fünf verschiedenen Kursen an vier verschiedenen Orten ausgeteilt (siehe Tabelle 1). Die Teilnahme an der Befragung war freiwillig. Die Teilnehmer hatten im Rahmen des Kurses ca. 10 Minuten Zeit den Fragebogen auszufüllen und abzugeben. Es stand Ihnen offen, sich mehr Zeit zu nehmen. Dies tat ein Teilnehmer der Kursweiterbildung auf Borkum. Er schickte den Bogen später per Post nach.

Tabelle 1: Verteilung der Fragebögen ( $n=117)$

\begin{tabular}{|c|l|l|c|}
\hline Datum & \multicolumn{1}{|c|}{ Ort } & \multicolumn{1}{|c|}{ Kurs } & $n$ \\
\hline 04.03.2010 & Gütersloh & Fallseminar Modul 3 & 20 \\
\hline 22.04.2010 & Wetzlar & Fallseminar Modul 2 & 24 \\
\hline 23.04.2010 & Gütersloh & Kursweiterbildung & 24 \\
\hline 13.05.2010 & Kassel & Fallseminar Modul 2 & 24 \\
\hline 03.06.2010 & Borkum & Kursweiterbildung & 25 \\
\hline
\end{tabular}

\subsection{Der Fragebogen}

Der Fragebogen zum Thema „Beratung zur PV - eine ärztliche Aufgabe?“ bestand aus sechs Frageblöcken.

\subsubsection{Angaben zur Person}

Im ersten Frageblock wurden die soziodemographischen Daten der Teilnehmer erfragt. Neben Alter und Geschlecht wurde nach der Facharztausbildung und dem Tätigkeitsbereich gefragt. Auch sollten die Befragten angeben, wie lange sie als Ärztin/Arzt tätig waren, und ob sie für sich selbst eine PV erstellt hatten.

\subsubsection{Aktueller Kenntnis- bzw. Informationsstand}

Der zweite Frageblock zielte darauf ab herauszufinden, wie gut die befragten Ärztinnen und Ärzte zum Thema PV informiert waren und woher sie Ihre Informationen zu diesem Thema bezogen hatten. Außerdem sollte der Wert einer PV im ärztlichen Alltag durch Ankreuzen von „stimme voll zu“, "stimme zu“, „stimme nicht $z u^{\prime}$ und „stimme überhaupt nicht zu“ eingeschätzt werden. Dieselbe Skala mit dem zusätzlichen Feld „weiß nicht" stand in der 
letzten Frage des zweiten Abschnitts zur Einschätzung zur Verfügung, ob das Gesetz vom September 2009 eine Hilfe im Umgang mit PV sei.

\subsubsection{Bisherige Beratungspraxis}

Im dritten Frageblock sollte herausgefunden werden, ob, wie und wie lange zum Thema PV beraten wurde. Die Teilnehmer konnten angeben, ob und wie häufig sie von Patienten auf das Thema PV angesprochen werden, und ob sich die Häufigkeit nach Beschluss des neuen Gesetzes zu Patentenverfügungen gesteigert hat. Von Interesse war auch, ob einer bestimmten Patientenklientel grundsätzlich zur Erstellung einer PV geraten wird und ob diese Patientenklientel von den behandelnden Ärztinnen und Ärzten dann auch darauf angesprochen wird. Schließlich wurde gefragt, ob von den Ärztinnen und Ärzten ein bestimmtes Formular empfohlen wird.

\subsubsection{Bedeutung der ärztlichen Beratung}

Der vierte Frageblock betraf den Stellenwert der ärztlichen Beratung zu PVen. Auch sollte festgestellt werden, bei welchen Schritten der Erstellung der Verfügung die ärztliche Beratung als wichtig angesehen wird.

In diesem Zusammenhang wurde auch gefragt, inwiefern eine PV, die ohne ärztliche Beratung erstellt wurde, überhaupt als eine Hilfestellung für das ärztliche Handeln angesehen wird. Auch wurden die Ärztinnen und Ärzte nach Ihrer Einschätzung gefragt, ob eine ärztliche Beratung zur PV verbindlich sein sollte.

\subsubsection{Kompetenz zur Beratung}

Im fünften Frageblock wurden die Ärztinnen und Ärzte gebeten, ihre eigene Kompetenz zur Beratung zu PVen einzuschätzen. Zudem wurden sie gefragt, ob sie Fortbildungen zu diesem Thema wünschen.

\subsubsection{Finanzierung von Beratung}

Der sechste Frageblock erfragte die mögliche Finanzierung der Beratung zur PV. Die Befragten wurden gebeten anzugeben, ob und wie sie Beratungsgespräche, sofern sie solche durchführen, abrechnen, und ob sie die bestehenden Abrechnungsmöglichkeiten für ausreichend einschätzen. Außerdem wurde gefragt, ob sie die Beratung zur PV eher als eine 
vom Patienten selbst zu finanzierende oder eine von der Solidargemeinschaft zu tragende Leistung betrachten.

Am Ende des Fragebogens gab es noch Raum für weitere Hinweise, Bemerkungen und Kommentare.

\subsection{Bearbeitung der Fragebögen und statistische Verfahren}

Die statistische Auswertung der erhaltenen Fragebögen erfolgte mittels des Softpakets Statistica 5.1 (StatSoft Inc, Tulsa, Oklahoma, USA) und dem Microsoft Office Tool Excel.

Gab es Differenzen bei stetigen Variablen, so wurden diese mit Hilfe des Mann-Whitney-UTests analysiert. Handelte es sich um dichotome oder kategoriale Daten, so wurden die Häufigkeiten in 2x2 Tabellen eingetragen. Durch den $\mathrm{Chi}^{2}$-Test wurde exakt nach Fischer auf Unterschiede geprüft. Für den P-Wert wurde bei allen statistischen Tests als Signifikanzgrenze kleiner als 0.05 festgelegt $(p<0.05)$. Alle Fragen, in denen die Möglichkeit bestand, eine Antwort frei zu formulieren, wurden separat ausgewertet. 


\section{Ergebnisse}

Von insgesamt 117 ausgeteilten Fragebögen wurden 117 ausgefüllt zurückgegeben. Dies entspricht einer Rücklaufquote von $100 \%$.

\subsection{Soziodemographische Daten}

Tabelle 2 gibt einen Überblick über die soziodemographischen Daten der Studienteilnehmer. Sie zeigt die Verteilung nach Alter, Geschlecht, Facharztausbildung, Tätigkeitsbereich und Tätigkeitsdauer. Ferner gibt sie an, wie viele der befragten Ärzte für sich selbst eine PV erstellt haben.

Tabelle 2: Soziodemographische Daten der befragten Ärzte $(n=117)$

\begin{tabular}{|l|ll|}
\hline Alter & \\
$<30$ Jahre & $n=0$ & $(0 \%)$ \\
31-40 Jahre & $n=26 \quad(22,2 \%)$ \\
41-50 Jahre & $n=47 \quad(40,2 \%)$ \\
51-60 Jahre & $n=39 \quad(33,3 \%)$ \\
$>60$ Jahre & $n=5 \quad(4,3 \%)$ \\
\hline $\begin{array}{l}\text { Geschlecht } \\
\text { Weiblich }\end{array}$ & $n=52 \quad(44,4 \%)$ \\
Männlich & $n=65 \quad(55,6 \%)$ \\
\hline
\end{tabular}

\begin{tabular}{|l|ll|}
\hline Facharztausbildung (mehr als eine Antwort möglich) & \\
Allgemeinmedizin & $\mathrm{n}=49 \quad(41,9 \%)$ \\
Innere Medizin & $\mathrm{n}=37 \quad(31,6 \%)$ \\
Chirurgie & $\mathrm{n}=6 \quad(5,1 \%)$ \\
Anästhesie & $\mathrm{n}=25 \quad(21,4 \%)$ \\
Sonstige & $\mathrm{n}=14 \quad(12,3 \%)$ \\
\hline Tätigkeitsbereich (mehr als eine Antwort möglich) & $\mathrm{n}=55 \quad(47 \%)$ \\
Krankenhaus & $\mathrm{n}=61 \quad(52,1 \%)$ \\
Niedergelassener Bereich & $\mathrm{n}=5 \quad(4,3 \%)$ \\
Sonstiges & \\
\hline
\end{tabular}




\begin{tabular}{|l|ll|}
\hline Tätigkeitsdauer & \\
Unter 5 Jahre & $n=3 \quad(2,7 \%)$ \\
5-10 Jahre & $n=22 \quad(18,8 \%)$ \\
11-20 Jahre & $n=41 \quad(35 \%)$ \\
Über 20 Jahre & $n=51 \quad(43,6 \%)$ \\
\hline Für sich selbst PV erstellt & \\
Ja & $n=32 \quad(27,6 \%)$ \\
Nein & $n=84 \quad(72,4 \%)$ \\
Keine Angaben & $n=1$ & $(0,9 \%)$ \\
\hline
\end{tabular}

Ein Großteil der Teilnehmer war zwischen 41 und 50 Jahre alt (40,2 \%). 37,6 \% waren über 50 Jahre alt, 22,2 \% 40 Jahre oder jünger. Der männliche Anteil bei den Studienteilnehmern war mit 55,6\% etwas größer als der weibliche Anteil (44.4\%).

Die meisten der befragten Ärzte waren Fachärzte der Allgemeinmedizin (41,9 \%). Fast ein Drittel hatte einen Facharzt der Inneren Medizin (31,6 \%), ein knappes Viertel der Anästhesie $(21,4 \%)$. Es gab nur wenige Fachärzte der Chirurgie (5,1\%). 12,3\% gaben an, eine andere Facharztausbildung zu haben.

Etwas mehr als die Hälfte der Teilnehmer kam aus dem niedergelassenen Bereich (52,1 \%). 47 \% waren im Krankenhaus tätig. Fünf Ärzte (4,3\%) gaben an, in anderen Bereichen (in einem Medizinisches Versorgungszentrum, beim Gesundheitsamt, beim MDK, freiberuflich und als Honorararzt) tätig zu sein.

Nur drei der befragten Ärzte waren weniger als fünf Jahre im ärztlichen Beruf tätig. Ein knappes Fünftel arbeitete 5-10 Jahre (18,8\%), etwa ein Drittel 11-20 Jahre als Arzt (35\%). Fast die Hälfte war zum Zeitpunkt der Befragung über 20 Jahre im Arztberuf tätig (43,6 \%).

Die große Mehrheit der befragten Ärzte hatte für sich keine PV erstellt (72,4\%). 32 Teilnehmer gaben an, für sich eine PV erstellt zu haben (27,6\%). Ein Teilnehmer machte hierzu keine Angabe. 


\subsection{Aktueller Informationsstand sowie allgemeine Einschätzung der Bedeutung von Patientenverfügungen für die ärztliche Praxis}

Die Fragen 2.1 bis 2.4 bezogen sich auf den aktuellen Informationsstand sowie auf die Einschätzung der Teilnehmer bezüglich der Bedeutung von PVen für die ärztliche Praxis.

\subsubsection{Wie gut fühlen sich die Ärzte informiert?}

Über 90 \% der befragten Ärzte gaben an, zum Thema PV gut informiert zu sein. Etwa jeder Zehnte fühlte sich weniger gut informiert (9,5\%). Ein Teilnehmer machte keine Angaben. (Abb. 1)

Abbildung 1: Stellungnahme zu der Aussage: „Ich bin über das Thema PV gut informiert.“

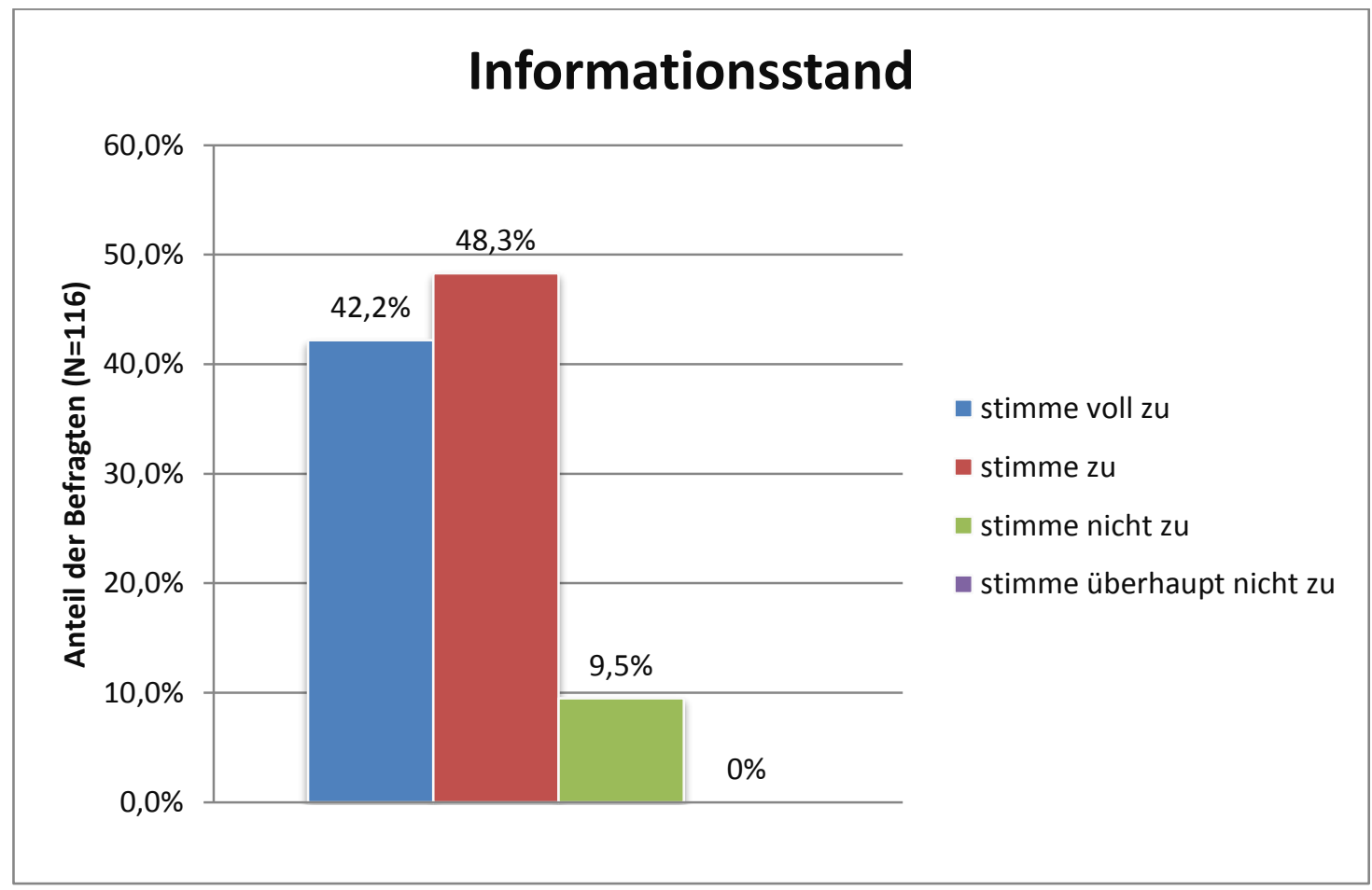




\subsubsection{Was hat zum Wissen der Ärzte beigetragen?}

Als wichtigste Informationsquellen zum Thema PV wurden mit je 82,1\% Fachmedien und Vorträge zum Thema genannt. Letztere schließen Fortbildungen mit ein. $62,4 \%$ der Teilnehmer bezogen ihr Wissen aus dem Austausch mit Kollegen, 42,7\% aus Berichten in den Massenmedien. Eigene Recherchen im Internet wurden von 28,2\% der Teilnehmer angegeben. Sonstige Quellen waren Texte der Ethikkommission sowie der praktische Umgang mit PVen. Themenspezifische Fortbildungen wurden von $6 \%$ der Teilnehmer noch einmal unter, Sonstige Quellen`aufgeführt. (Abb. 2)

Abbildung 2: Angaben dazu, welche Medien zum Wissenstand beigetragen haben.

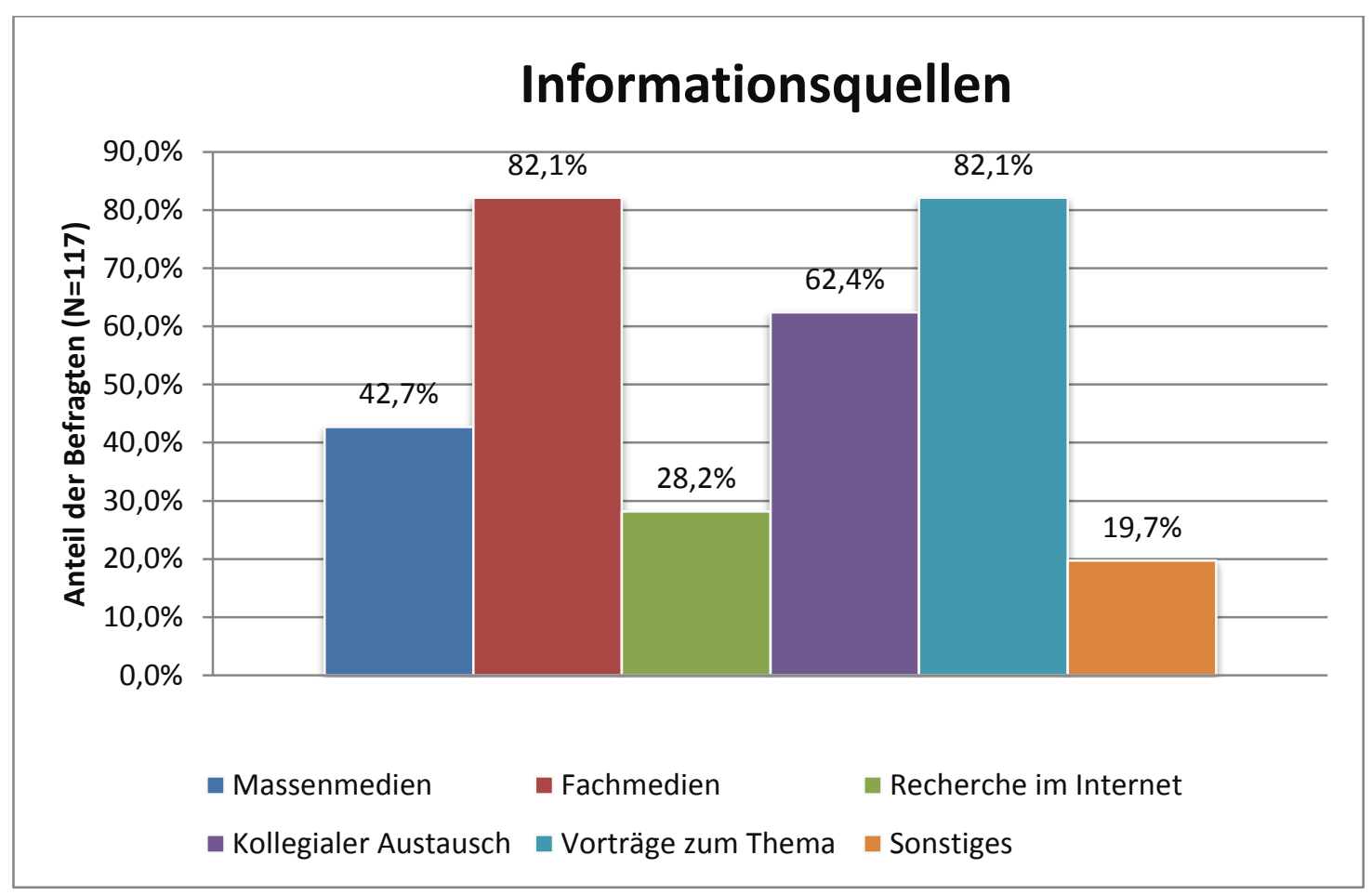




\subsubsection{Vereinfacht eine Patientenverfügung die Entscheidungsfindung?}

Der Aussage, dass eine PV die Entscheidungsfindung in Bezug auf ärztliche Maßnahmen bei nicht mehr einwilligungsfähigen Patienten vereinfacht, stimmte eine große Mehrheit von $87,1 \%$ der befragten Ärzte zu. Fast die Hälfte stimmte der Aussage sogar voll zu. Ungefähr ein Achtel der Befragten stimmte nicht zu, wobei nur einer überhaupt nicht zustimmte. Eine Person machte dazu keine Angaben. (Abb. 3)

Abbildung 3: Stellungnahme zu der Aussage: „Eine PV vereinfacht die Entscheidungsfindung bei nicht mehr einwilligungsfähigen Patienten."

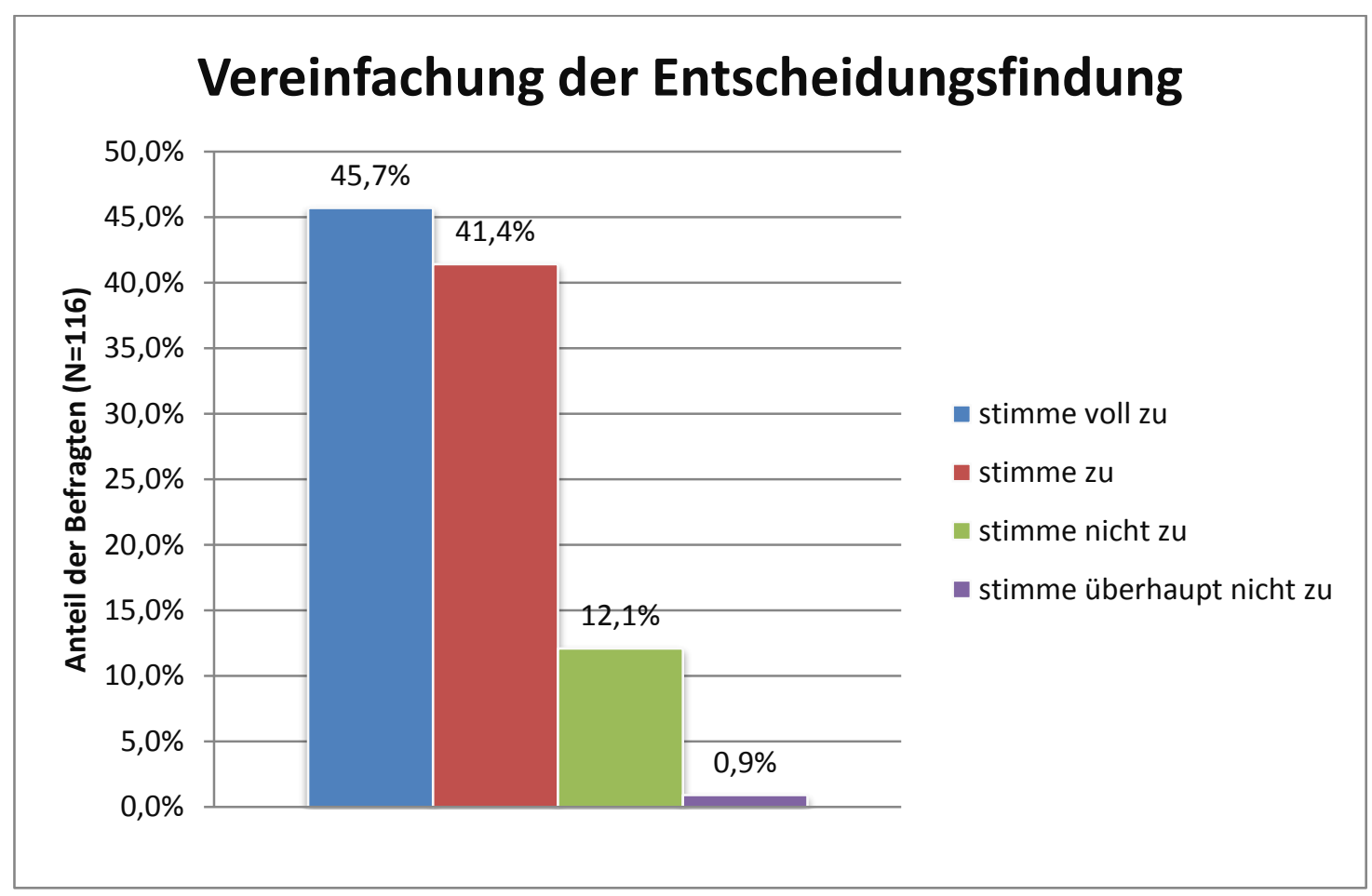




\subsubsection{Ist das neue Gesetz eine Hilfe im Umgang mit Patientenverfügungen?}

Drei Viertel der befragten Ärzte sahen in dem neuen Gesetz zur PV eine Hilfe im Umgang mit PVen (79,1 \%). Für etwa jeden zehnten stellte das Gesetz keine Hilfe dar (9,5 \%). Die übrigen wussten diese Frage nicht eindeutig zu beantworten, zwei machten keine Angaben. (Abb. 4)

Abbildung 4: Stellungnahme zu der Aussage: "Das neue Gesetz vom 1. September 2009 ist eine Hilfe im Umgang mit PV."

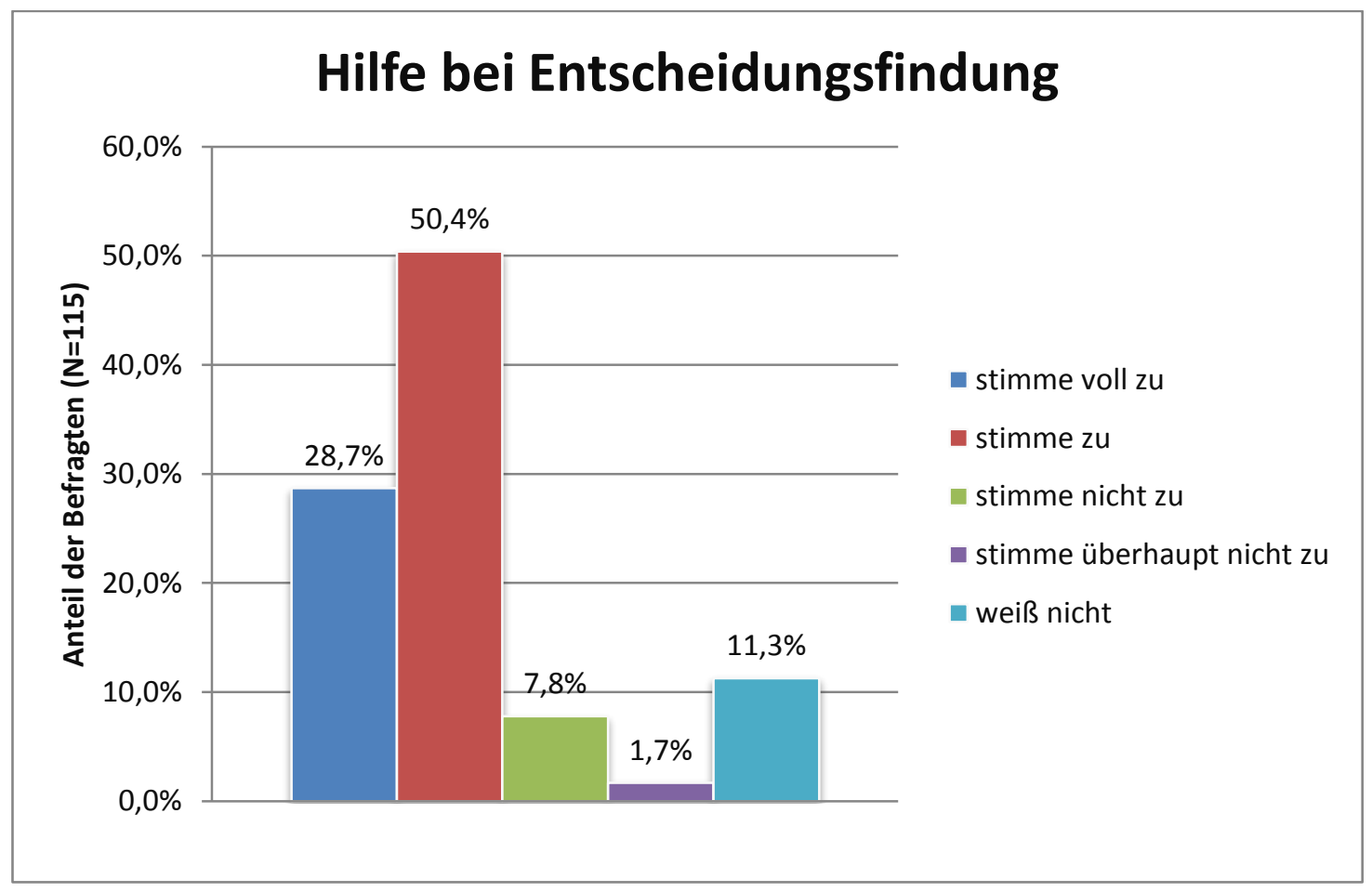




\subsection{Bisherige Beratungspraxis}

Die Fragen 3.1 bis 3.8 bezogen sich auf die bisherige Praxis der Beratung zu PVen.

\subsubsection{Wie häufig werden Ärzte auf das Thema Patientenverfügung angesprochen?}

Zwei Drittel der befragten Ärzte gaben an, regelmäßig - d.h. monatlich (36,8 \%), wöchentlich $(30,8 \%)$ oder gar täglich $(3,4 \%)$ - von Patienten auf das Thema PV angesprochen zu werden. Die Übrigen wurden seltener $(27,4)$ oder noch nie $(1,7 \%)$ auf das Thema angesprochen. (Abb. 5)

Abbildung 5: Wie oft werden die befragten Ärzte auf das Thema PV angesprochen?

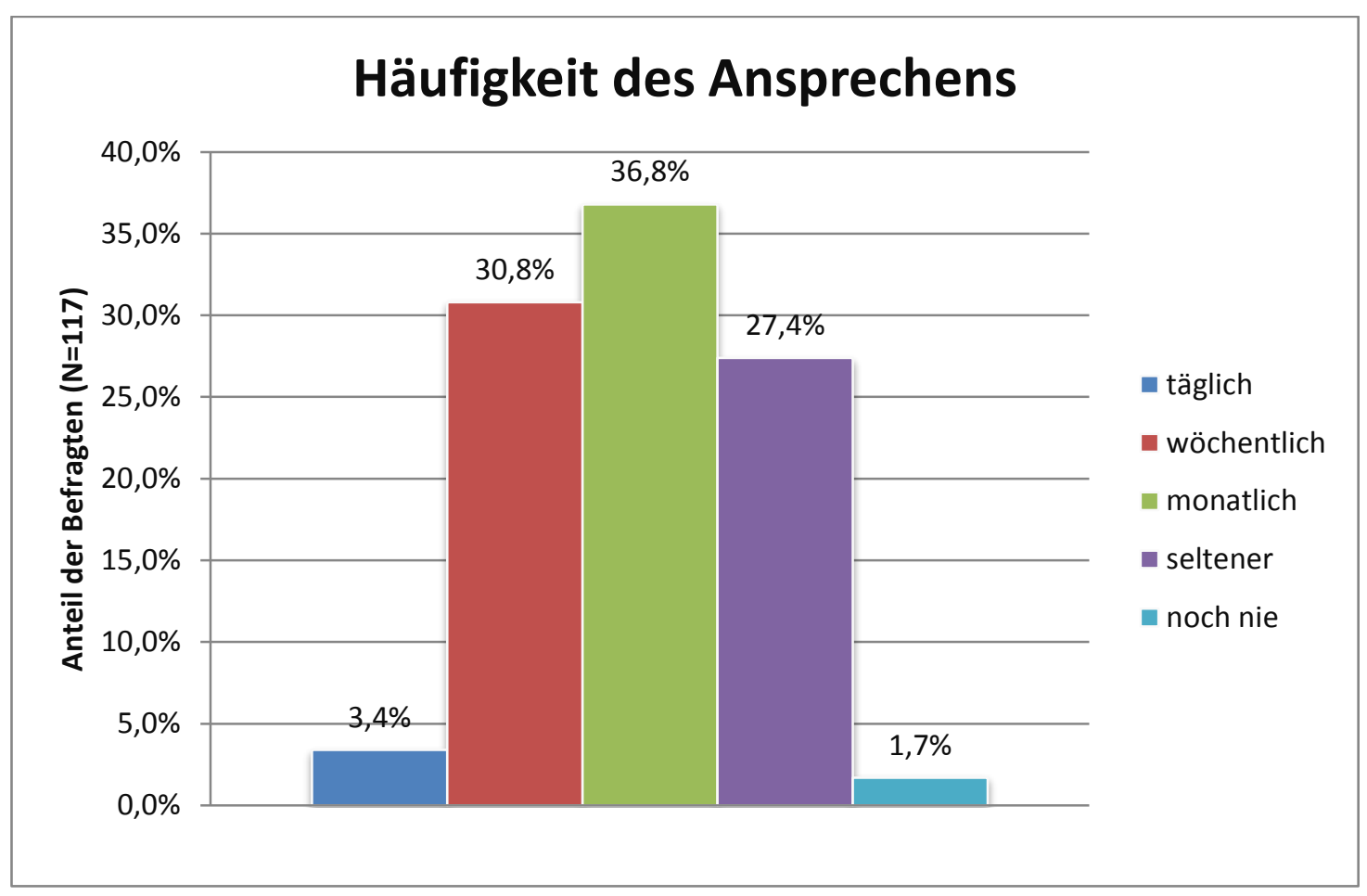




\subsubsection{Hat das neue Gesetz zu mehr Anfragen geführt?}

Über ein Drittel der Studienteilnehmer gab an, dass das Gesetz zur PV dazu geführt hat, dass sie nun häufiger auf das Thema PV angesprochen werden (38,9 \%). Knapp zwei Drittel sahen keine Veränderung (61,1\%). Bei keinem hatte sich die Häufigkeit der Anfragen verringert. Vier Ärzte machten keine Angaben. (Abb. 6)

Abbildung 6: Werden die befragten Ärzte seit Erlass der neuen Regelung häufiger angesprochen?

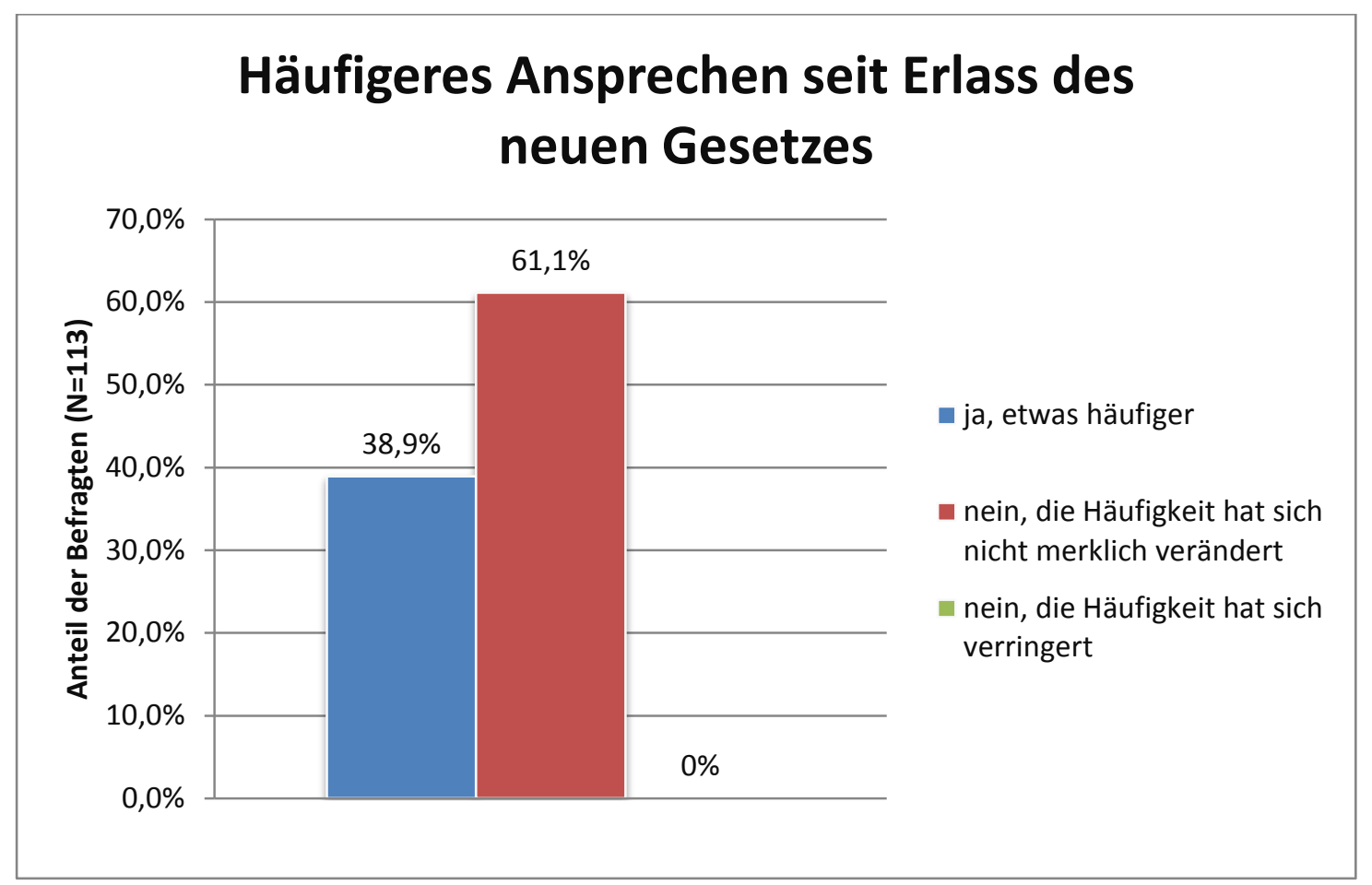




\subsubsection{Welche Ärzte beraten zum Thema Patientenverfügung?}

Drei Viertel der Ärzte gaben an, ihre Patienten zum Thema PV zu beraten (73,3 \%). 26,8 \% taten dies nicht. Einer machte keine Angabe. (Abb. 7)

Abbildung 7: Wird zum Thema PV beraten?

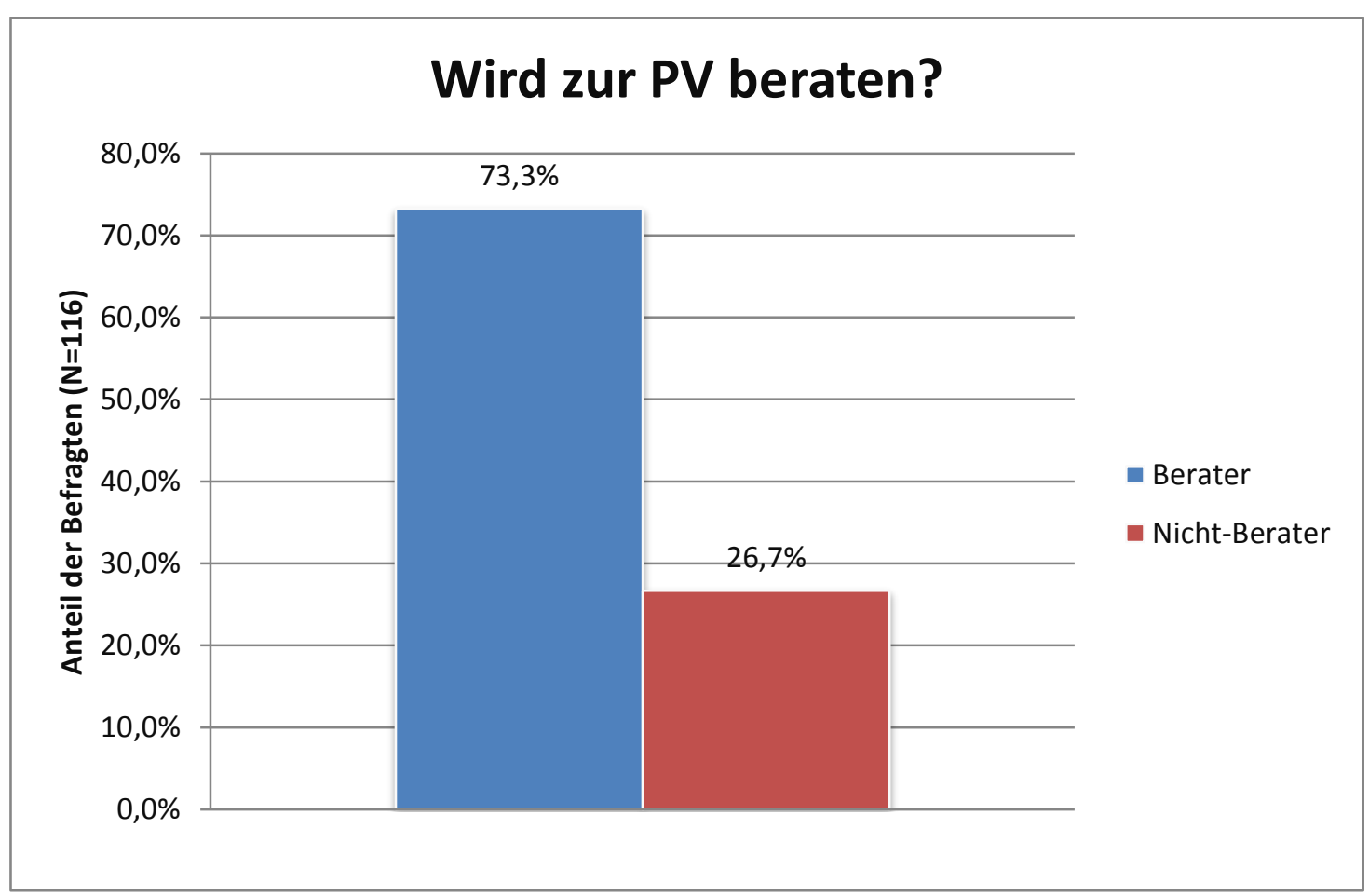

Analysiert man die soziodemografischen Daten der Ärzte, die zum Thema PV beraten, so lassen sich einige signifikante Unterschiede feststellen: Männer berieten signifikant häufiger als Frauen (62,4 \% vs. 37,7\%), niedergelassene Ärzte häufiger als ihre klinischen Kollegen (63,5\% vs. $38,8 \%)$ und Fachärzte für Allgemeinmedizin häufiger als anderen Fachärzte (Allgemeinmediziner: 91,8\%, Internisten: $73 \%$, Anästhesisten: $50 \%$, Sonstige: 35,7\%, Chirurgen: 16,1\%).Unter den Beratern gab es auch deutlich mehr Ärzte, die für sich selbst eine PV erstellt hatten (33,3\%), als bei den Nicht-Beratern (12,9\%). Bei den Kategorien Alter und Tätigkeitsdauer konnten hingegen keine signifikanten Unterschiede festgestellt werden. (vgl. Tabelle 3, Seite 53)

Betrachtet man die Fragen zum aktuellen Kenntnis- und Informationszustand im Kontext zur Beratungspraxis, so stellt sich auch hier ein signifikanter Zusammenhang dar: Ärzte, die nicht zu PVen beraten, geben deutlich häufiger an, sich nicht gut zu diesem Thema informiert zu fühlen, als beratende Ärzte (23,3 \% vs. 4,7\%). 
Bezogen auf die Fragen, ob eine PV die Entscheidungsfindung bei nicht mehr einwilligungsfähigen Patienten vereinfacht und ob das neue Gesetz eine Hilfe im Umgang mit PVen ist, ergaben sich keine Signifikanzen.

In Bezug auf die bisherige Beratungspraxis wird deutlich, dass Ärzte, die zur PV beraten, häufiger angaben, auf das Thema PV angesprochen zu werden. Monatlich werden $80 \%$ der beratenden Ärzte auf das Thema angesprochen, dagegen nur weniger als die Hälfte der nicht beratenden Ärzte $(45,1 \%)$.

Zudem ergab sich ein signifikanter Zusammenhang bezogen auf die Frage, ob sich die Anfragen zu PVen seit Erlass der neuen Regelung gesteigert haben.

Während nur etwa ein Fünftel der nicht beratenden Ärzte angab, dass sich die Häufigkeit der Nachfrage etwas gesteigert hat (22,2\%), gab dies fast die Hälfte der beratenden Ärzte an $(43,5 \%)$. Beratende Ärzte behaupteten also signifikant häufiger, dass sich die Nachfragen zum Thema PV nach Erlass des neuen Gesetztes gesteigert hätten.

\subsubsection{Durchschnittliche Beratungsdauer}

Die Dauer der Beratungsgespräche variierte zwischen 5 und 60 Minuten. Die durchschnittliche Beratungsdauer - berechnet aus den Mittelwerten der angegebenen Zeitspannen - betrug 21 Minuten. Ein Arzt gab an, ein Aufklärungsgespräch immer in drei Zeiteinheiten zu je 10-15 Minuten aufzuteilen. Drei Ärzte, die zum Thema PV beraten, machten keine Zeitangabe.

Es wurde kein signifikanter Unterschied zwischen der Beratungsdauer und dem Tätigkeitsort festgestellt. Die Beratungsdauer ist demnach im niedergelassenen Bereich ähnlich wie in der Klinik.

\subsubsection{Inhalte einer Beratung zur Patientenverfügung}

Die Mehrheit der beratenden Ärzte klärte über das vom Patienten gewünschte Procedere in möglicherweise auftretenden Situationen auf (65,8 \%). Je 64,1\% der Ärzte gaben dem Patienten allgemeine Informationen zum Thema PV bzw. klärten über mögliche Situationen auf, in denen eine PV zur Anwendung kommen kann. Fast genauso viele gaben allgemeine Informationen zur VV (62,4 \%). 45,3\% der Teilnehmer gaben dem Patienten Hinweise auf weitere Informationsmöglichkeiten. Nur etwa ein Drittel sprach mit den Patienten auch über 
die rechtlichen Aspekte (34,2 \%). Etwa ein Zehntel (9,4 \%) kreuzte „Sonstiges“ an und nannte als Beispiele die Gabe von weiterführenden Informationen (3,5\%), konkrete Angaben über z.B. PEG/Beatmung und Hinweise zur BV (je 2,4 \%). (Abb. 8)

Abbildung 8: Inhalte einer Beratung zur PV.

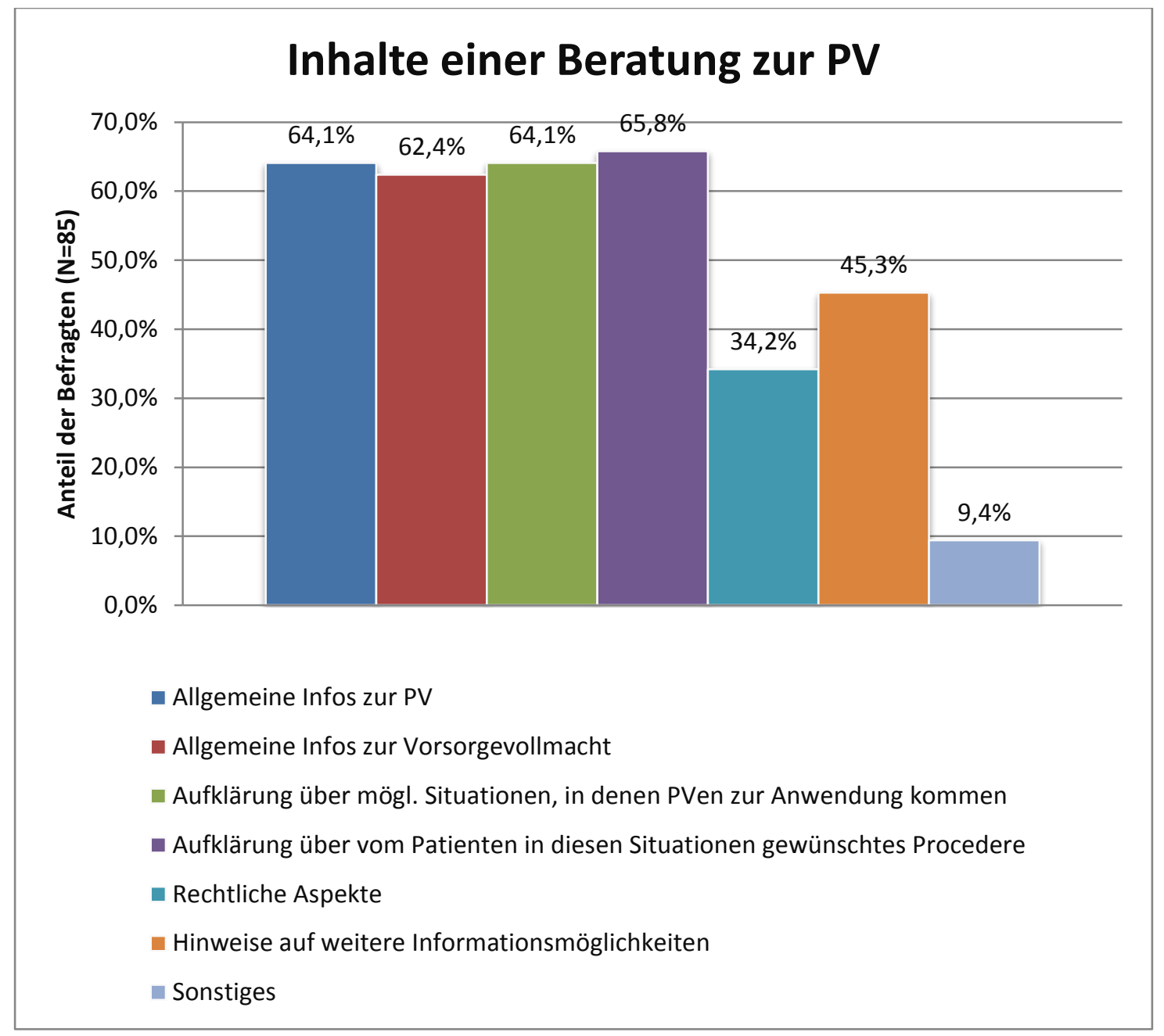




\subsubsection{Empfehlen die Teilnehmer ein bestimmtes Formular?}

Gut ein Drittel der Berater empfahl ein bestimmtes Formular (34,9\%). Besonders häufig genannt wurde die „Vorlage der Bayerischen Landesärztekammer" (17,2 \%) (da die Bayerische Landesärztekammer keine Vorlage für eine PV gestaltet hat, kann man annehmen, dass das Formular des Bayerischen Staatsministeriums der Justiz gemeint ist) und die Christliche PV (ebenfalls 17,2 \%). Jeder zehnte empfahl eine selbst erstellte Vorlage (10,3 \%). Zwei Ärzte, die zur PV beraten, machten keine Aussage zu dieser Frage. (Abb. 9)

Abbildung 9: Wird ein bestimmtes Formular empfohlen?

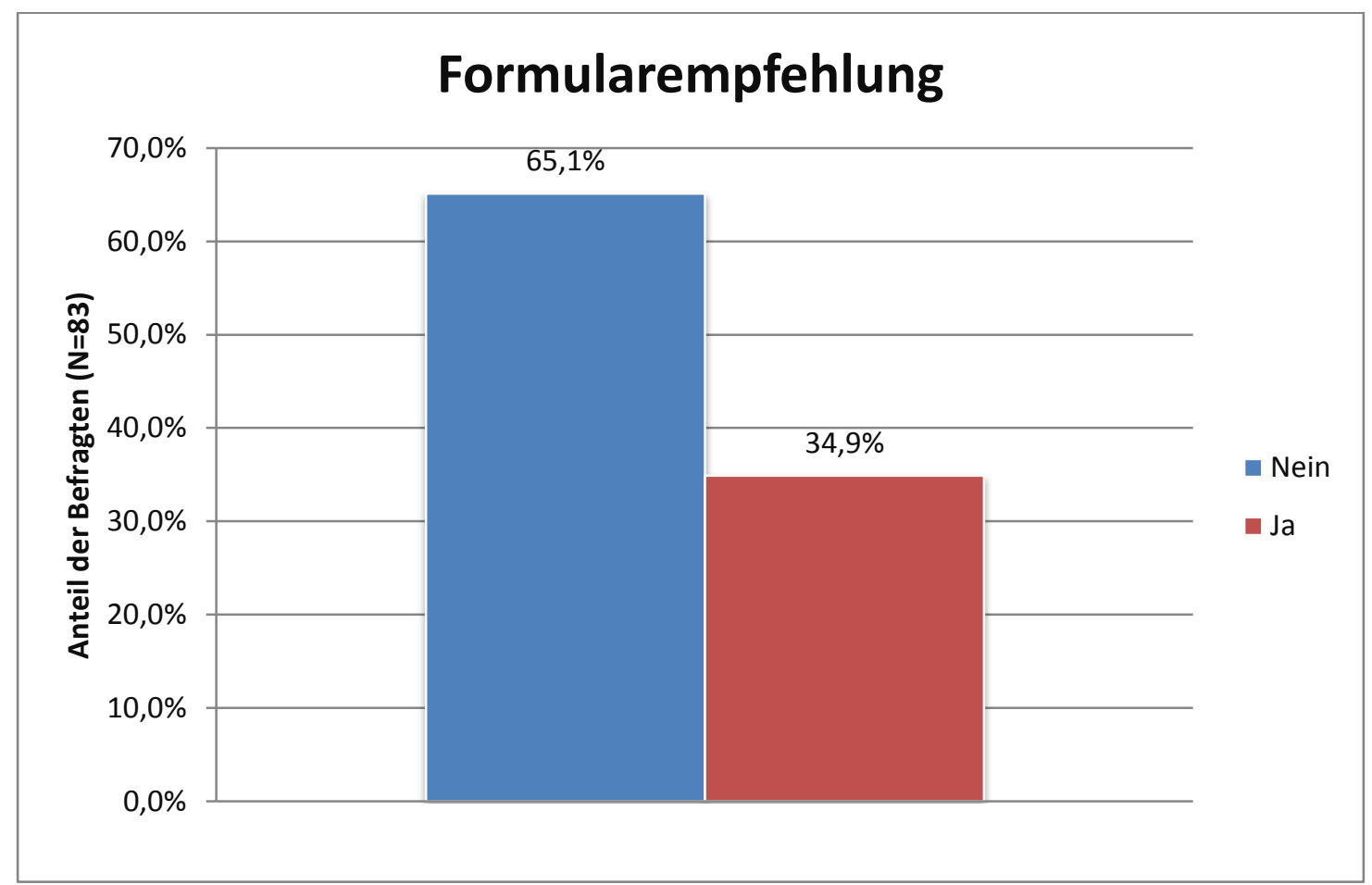




\subsubsection{Sprechen Ärzte Patienten auf die Möglichkeit einer Patientenverfügung an?}

Gut drei Viertel der Ärzte sprechen ihre Patienten auf die Möglichkeit einer PV an (73,5 \%), drei Teilnehmer machten keine Angaben.

Am häufigsten wurden Patienten mit infauster Prognose auf die Möglichkeit einer PV hingewiesen (84,9\%), gefolgt von den chronisch Kranken $(65,1 \%)$ und den älteren Patienten (50\%). 18,6 \% der Ärzte kreuzten die Antwortoption „Sonstiges“ an und nannten als Beispiel sporadische, situationsabhängige Aufklärung (3,5\%), Aufklärung Alleinstehender und Aufklärung vor operativen Eingriffen (je 2,3 \%). 15,1 \% der Ärzte wiesen alle Patienten auf die Möglichkeit, eine PV zu erstellen, hin. (Abb. 10)

Abbildung 10: Wen sprechen die befragten Ärzte auf die Möglichkeit einer PV an?

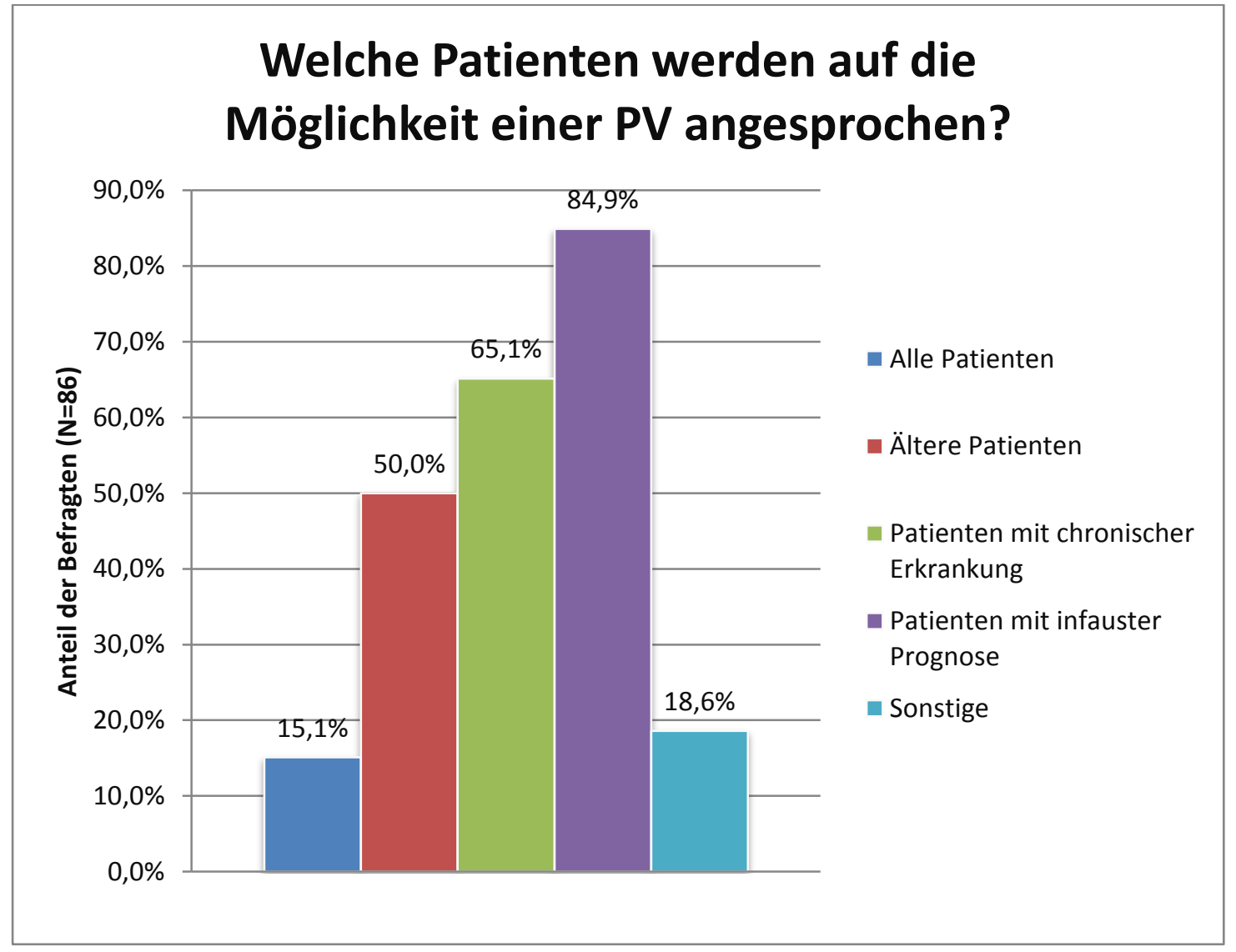




\subsubsection{Empfehlen die teilnehmenden Ärzte ihren Patienten die Erstellung einer Patientenverfügung?}

Fast alle an der Studie teilnehmenden Ärzte gaben an, die Erstellung einer PV zu empfehlen (89,7 \%). Zwei Teilnehmer machten keine Angaben.

Patienten mit einer infausten Prognose bildeten die Patientengruppe, denen am häufigsten die Erstellung einer PV nahelegt wurde (84,8\%), gefolgt von den Gruppen der chronisch Kranken (73,3\%) und der älteren Patienten (71,4\%). 43,8\% der Ärzte legten die Erstellung einer PV allen ihren Patienten nahe. "Sonstiges“ wählten 18,1\% der Teilnehmer; als Beispiele wurden Empfehlungen auf Anfrage, abhängig von der Lebenssituation (je 3,8 \%) oder vor operativen Eingriffen (2,9\%) genannt.

Männliche Ärzte empfahlen signifikant häufiger die Erstellung einer PV als ihre weiblichen Kollegen (60\% vs. $40 \%$ ). Andere signifikanten Zusammenhänge in Bezug auf die soziodemographischen Daten konnten nicht gefunden werden. Es zeigte sich aber ein signifikanter Zusammenhang bezogen auf die Frage, ob die Patienten auf die Möglichkeit einer PV angesprochen werden: 98,8 \% der Ärzte, die auf PVen hinweisen, legen ihren Patienten auch nahe, eine solche zu erstellen. (Abb. 11)

Abbildung 11: Wem empfehlen die befragten Ärzte die Erstellung einer PV?

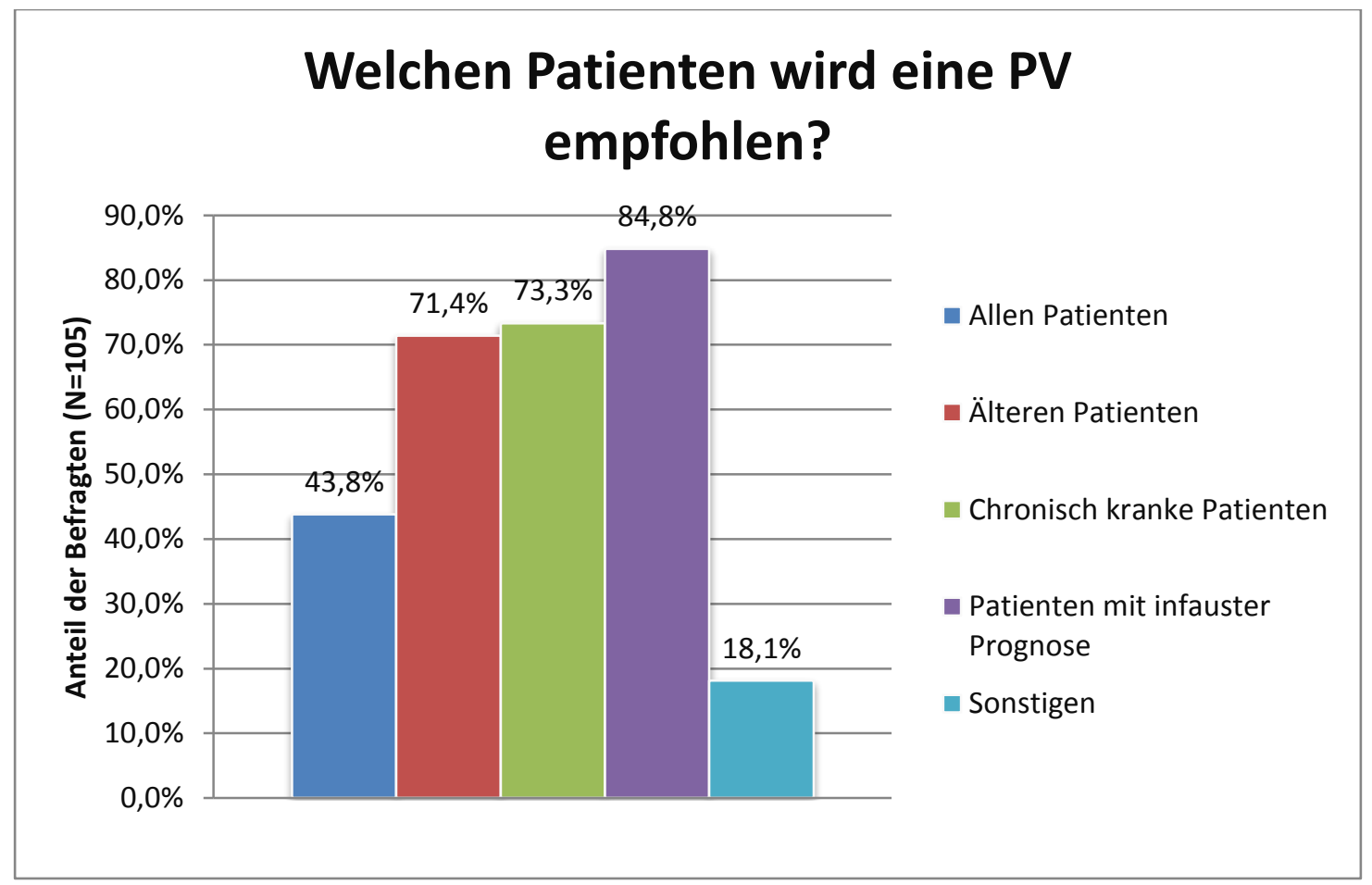




\subsection{Bedeutung der ärztlichen Beratung}

Die Fragen 4.1 bis 4.5 bezogen sich auf die Bedeutung von ärztlicher Beratung bei der Erstellung einer PV.

\subsubsection{Verständnis der Behandlungssituation}

Über vier Fünftel der befragten Ärzte stimmten der Aussage zu, dass der Patient ohne ärztliche Beratung die in seiner PV angeführten Behandlungssituationen nicht angemessen verstehen kann (82\%). 58,1\% der Ärzte stimmten sogar voll zu. Diese Einschätzung war unabhängig davon, ob die Ärzte Beratung zur PV durchführen oder nicht. (Abb.12)

Abbildung 12: Stellungnahme zu der Aussage: „Ohne ärztliche Beratung kann der Patient die möglichen Behandlungssituationen, auf die er in seiner PV Bezug nimmt, nicht angemessen verstehen.“

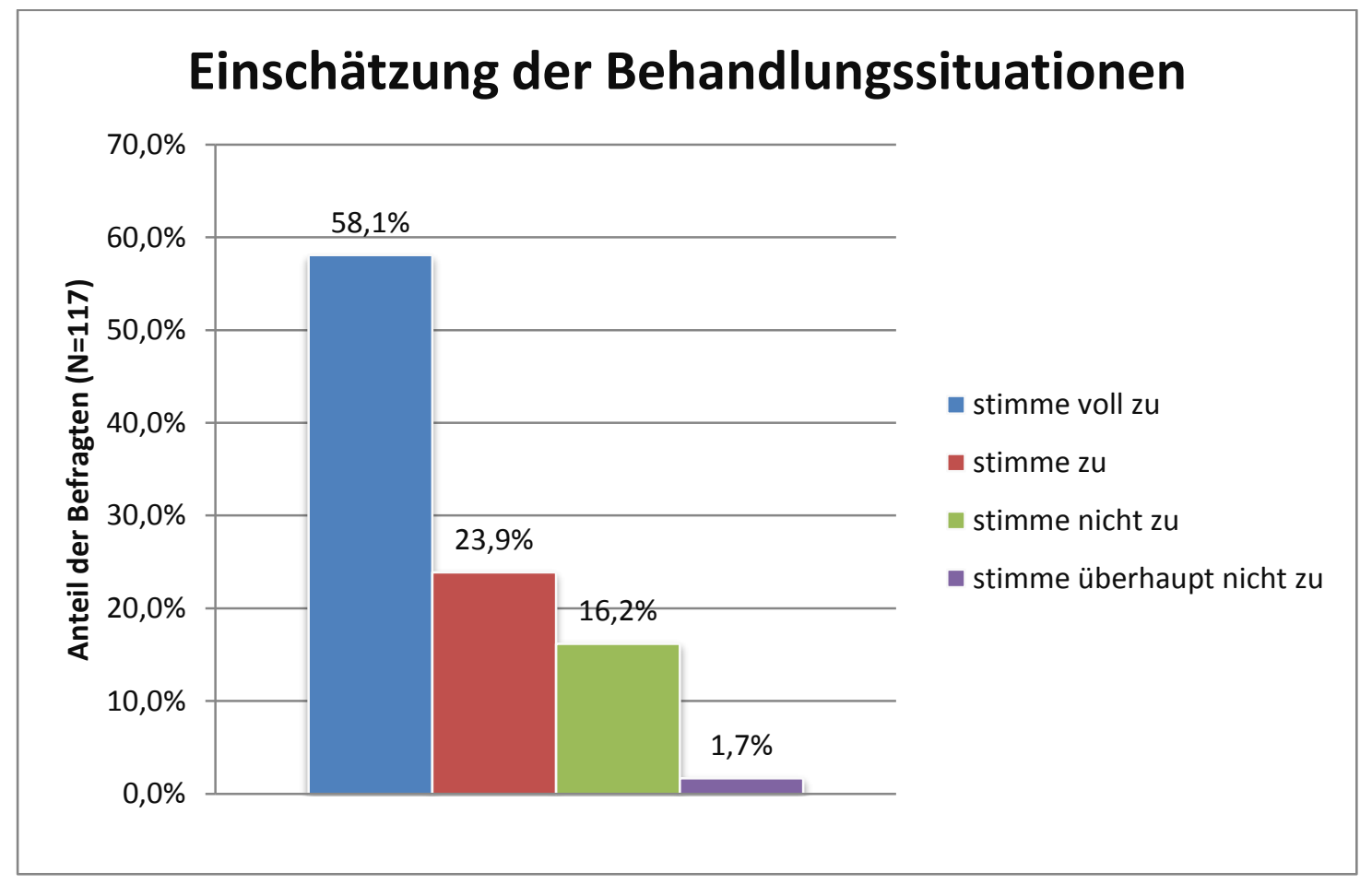




\subsubsection{Einschätzung von Schaden und Nutzen ärztlicher Maßnahmen}

Eine große Mehrheit der an der Umfrage teilnehmenden Ärzte stimmte der Aussage zu, dass der Patient den möglichen Nutzen und Schaden der ärztlichen Maßnahmen, zu denen er sich in einer PV äußert, nicht angemessen einschätzen kann (84,7\%). Auch hier konnte kein signifikanter Unterschied im Antwortverhalten zwischen Beratern und Nicht-Beratern festgestellt werden. (Abb. 13)

Abbildung 13: Stellungnahme zu der Aussage: "Ohne ärztliche Beratung kann der Patient den möglichen Nutzen und Schaden der ärztlichen Maßnahmen, zu denen er sich in einer PV äußert, nicht angemessen einschätzen."

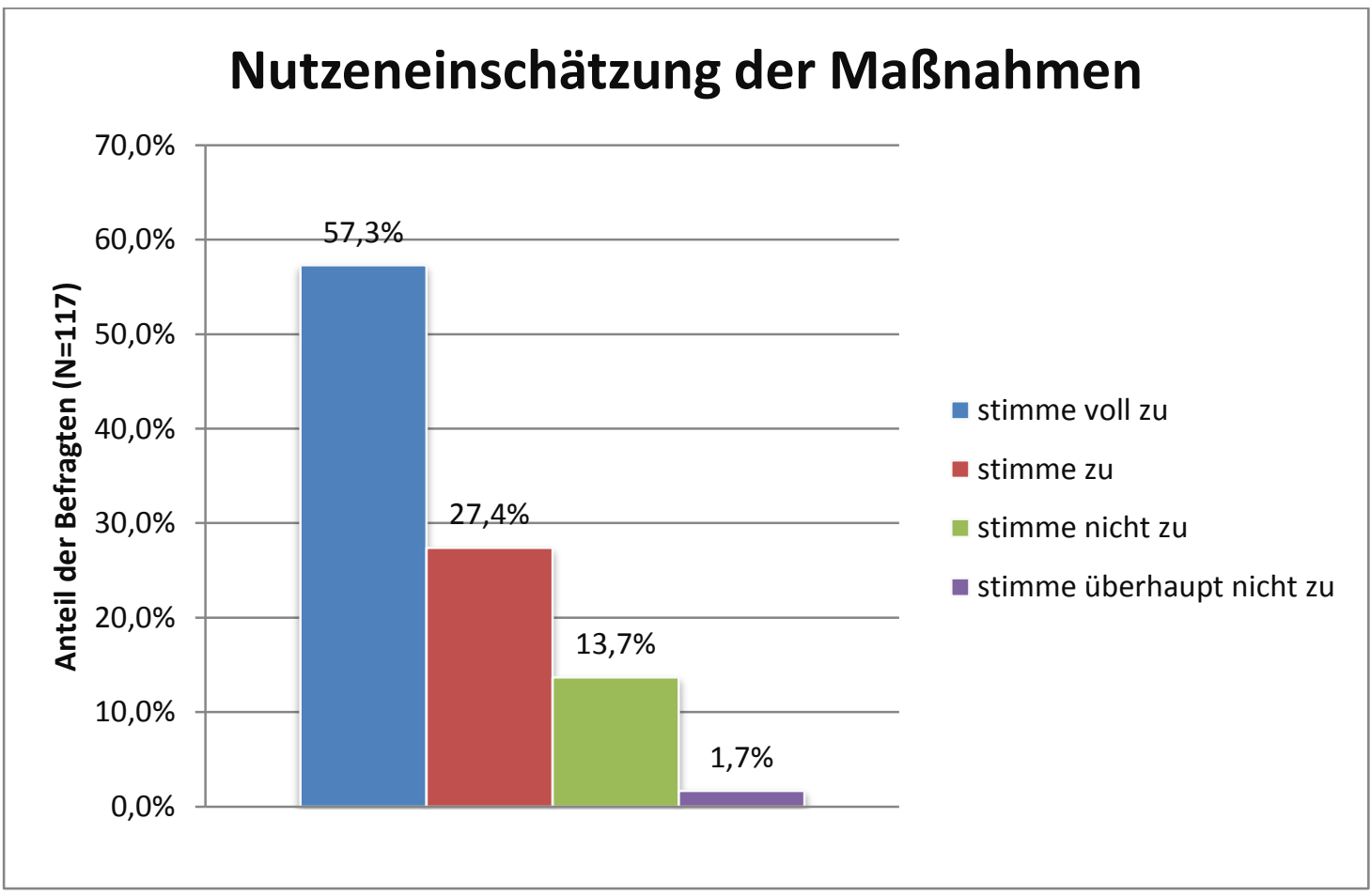




\subsubsection{Formulierung des tatsächlichen Patientenwillens}

Der Aussage, dass ohne ärztliche Beratung die Gefahr bestehe, dass der Wortlaut einer PV nicht mit dem tatsächlichen Willen des Patienten übereinstimmt, stimmten über drei Viertel der befragten Ärzte zu (78,6 \%). Berater stimmten der Aussage signifikant häufiger zu, als Nicht-Berater (82,3\% vs. 67,7\%). (Abb. 14)

Abbildung 14: Stellungnahme zu der Aussage: "Ohne ärztliche Beratung besteht die Gefahr, dass der Wortlaut der PV nicht mit dem tatsächlichen Willen des Patienten übereinstimmt."

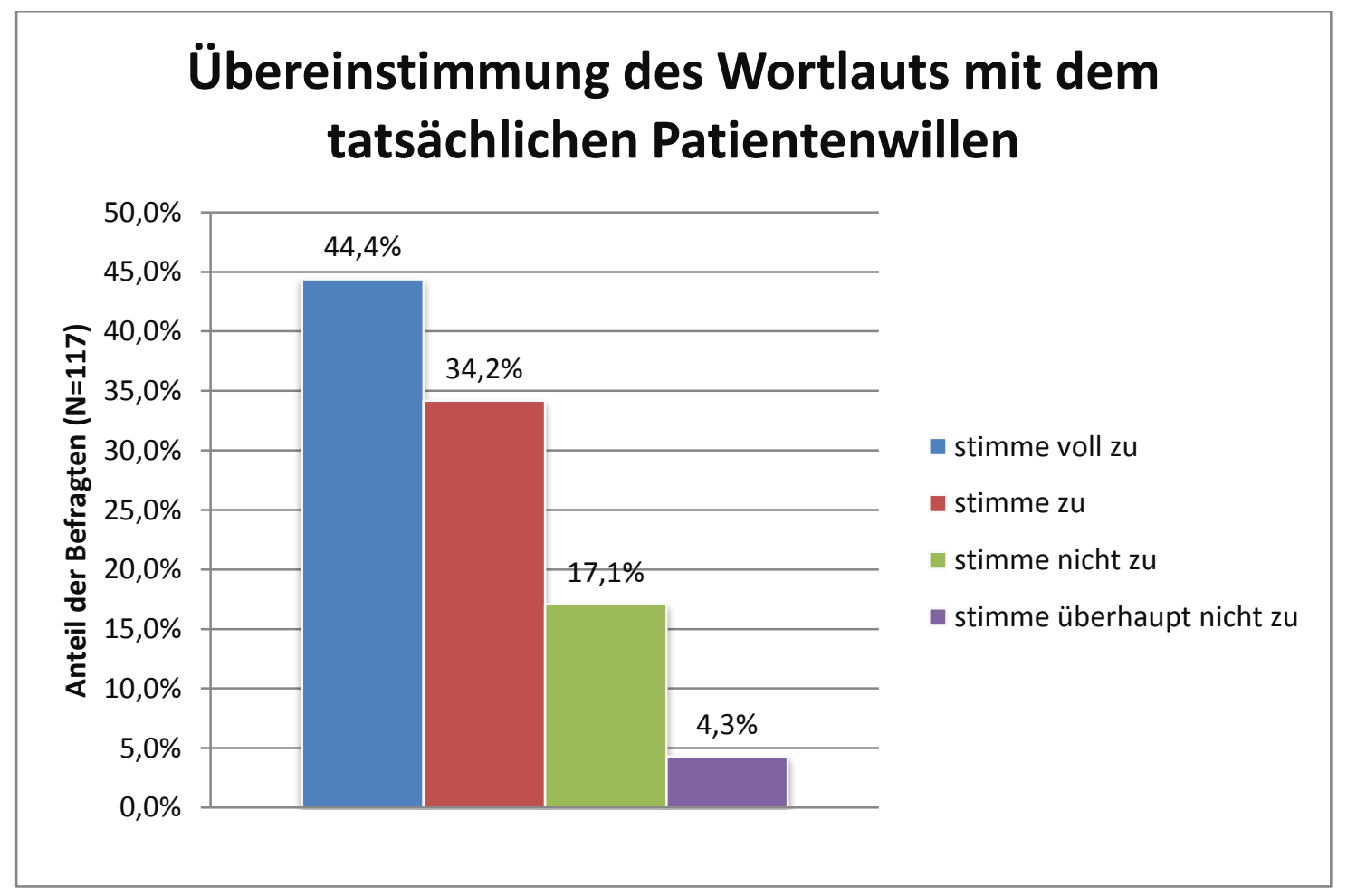




\subsubsection{Hilfestellung für den später behandelnden Arzt}

Etwa die Hälfte der befragten Ärzte stimmte der Aussage zu, dass eine ohne ärztliche Beratung erstellte PV keine Hilfestellung für den Arzt sei, der später mit der Verfügung konfrontiert wird (51,7\%). Signifikante Unterschiede zwischen Beratern und Nicht-Beratern bestehen nicht. (Abb. 15)

Abbildung 15: Stellungnahme zu der Aussage: „Ohne ärztliche Beratung ist die PV keine Hilfestellung für den Arzt, der später mit dieser konfrontiert wird."

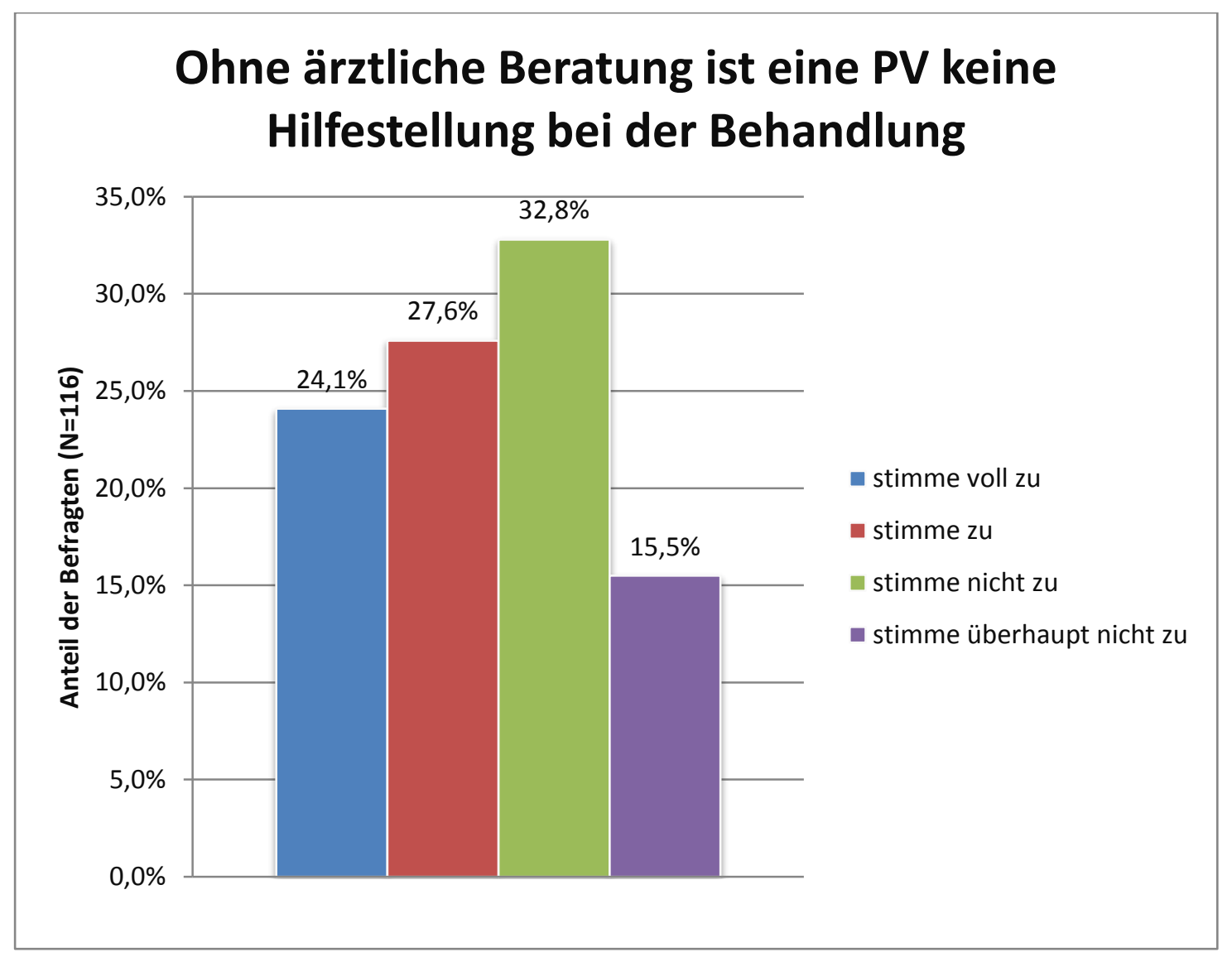




\subsubsection{Verbindliche ärztliche Beratung zur Erstellung einer Patientenverfügung}

Der Aussage, dass eine ärztliche Beratung zu PVen verbindlich sein sollte, stimmte über die Hälfte der befragten Ärzte zu (60\%).

31,3\% der Studienteilnehmer stimmten dem sogar voll zu. Berater stimmten der Aussage signifikant häufiger zu als Nicht-Berater. (Abb. 16)

Abbildung 16: Stellungnahme der befragten Ärzte, ob ärztliche Beratung zur PV verbindlich sein sollte.

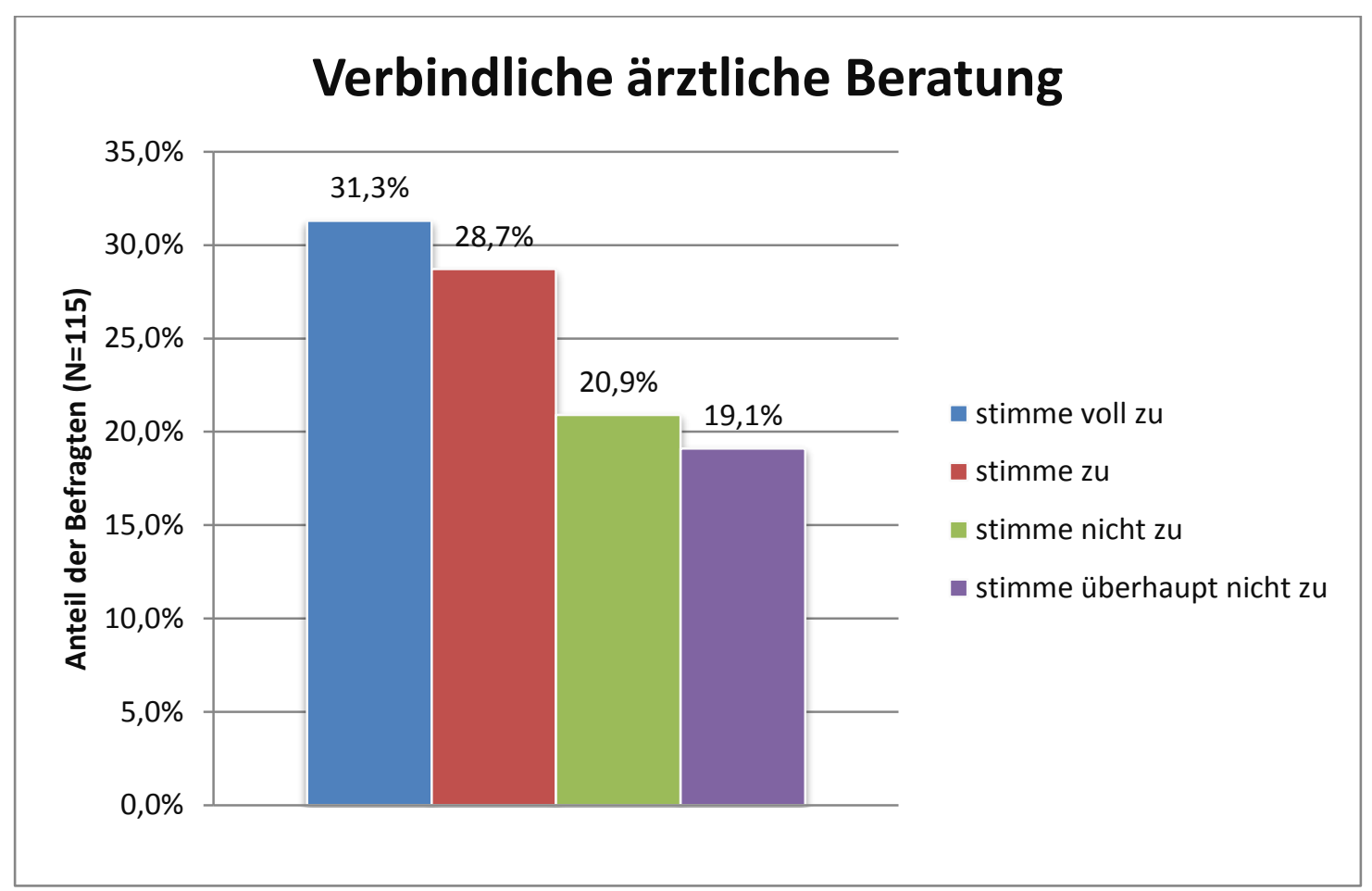




\subsection{Kompetenz zur Beratung}

Die Fragen 5.1 und 5.2 bezogen sich auf die subjektive Einschätzung der eigenen Beratungskompetenz und auf den Wunsch nach Fortbildungsmöglichkeiten zum Thema PV.

\subsubsection{Selbsteinschätzung der Beratungskompetenz}

Die große Mehrheit der befragten Ärzte fühlte sich ausreichend kompetent, um Patienten angemessen zu PVen beraten zu können (84,6\%). Ein Drittel der Teilnehmer stimmte der Aussage sogar voll zu $(33,3 \%)$.

Beratende Ärzte schätzten sich signifikant häufiger als ausreichend kompetent ein als NichtBerater: Während sich ungefähr zwei Drittel der nicht beratenden Ärzte kompetent genug fühlten (67,8\%), fühlten sich über $90 \%$ der beratenden Ärzte ausreichend kompetent (90,6\%). (Abb. 17)

Abbildung 17: Stellungnahme zu der Aussage, ob sich die befragten Ärzte ausreichend kompetent fühlen, Patienten angemessen zur PV zu beraten.

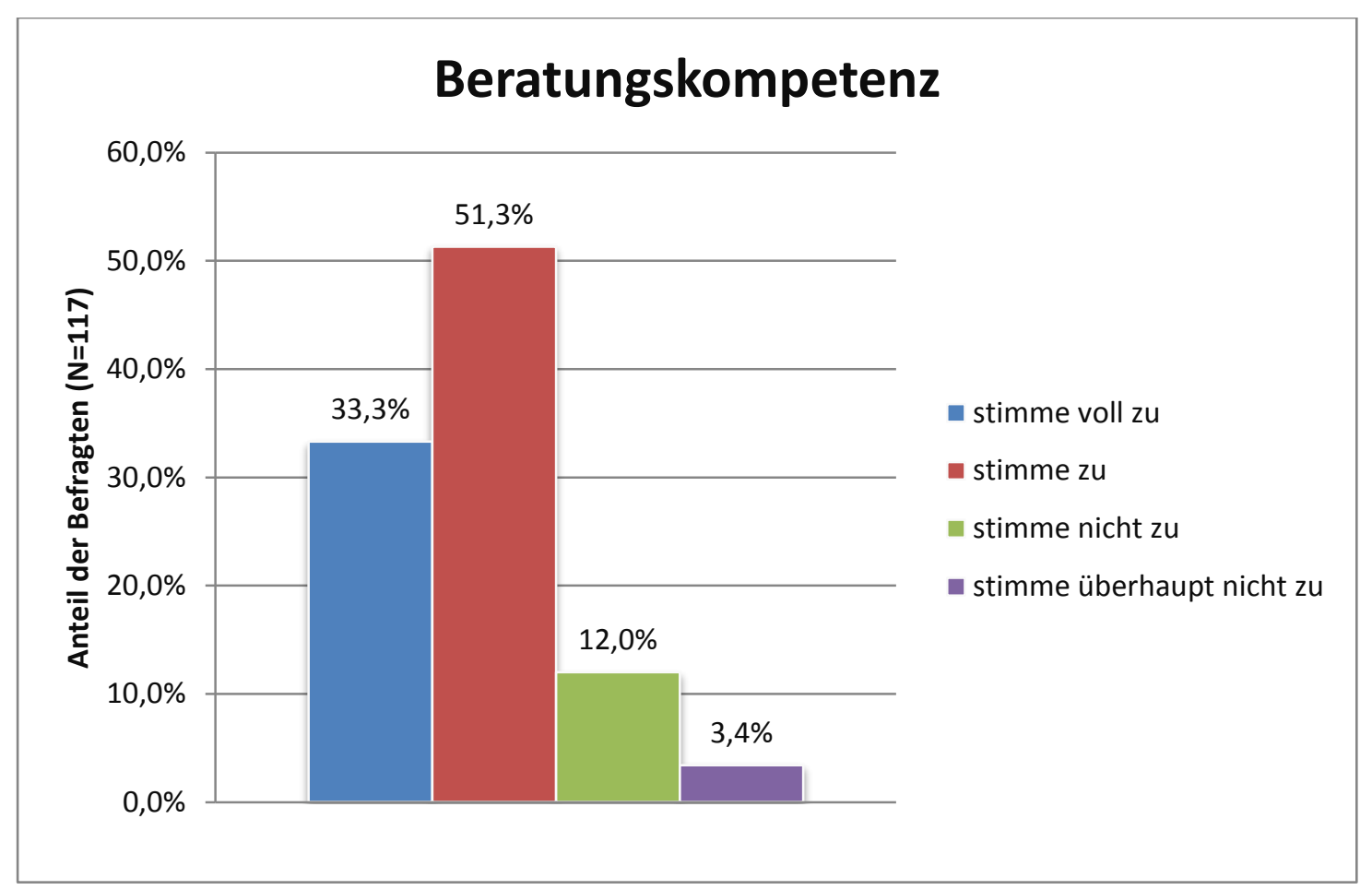




\subsubsection{Wird Fortbildung gewünscht?}

Fast die Hälfte der befragten Ärzte wünschte sich Fortbildung zum Thema PV, um Patienten angemessen beraten zu können (44,4\%). Gut ein Zehntel hätte gerne Fortbildungen zu den Rechtsgrundlagen (11,1\%). Einige möchten Formulierungshilfen (3,4\%). 34,8\% der Ärzte wünschte keine Fortbildung. Ein Fünftel entschied sich für die Antwortmöglichkeit „weiß nicht" $(20,9 \%)$.

Zwei Ärzte machten keine Angaben. Signifikante Unterschiede im Antwortverhalten zwischen Beratern und Nicht-Beratern bestanden nicht. (Abb. 18)

Abbildung 18: Werden Fortbildungen zum Thema PV gewünscht?

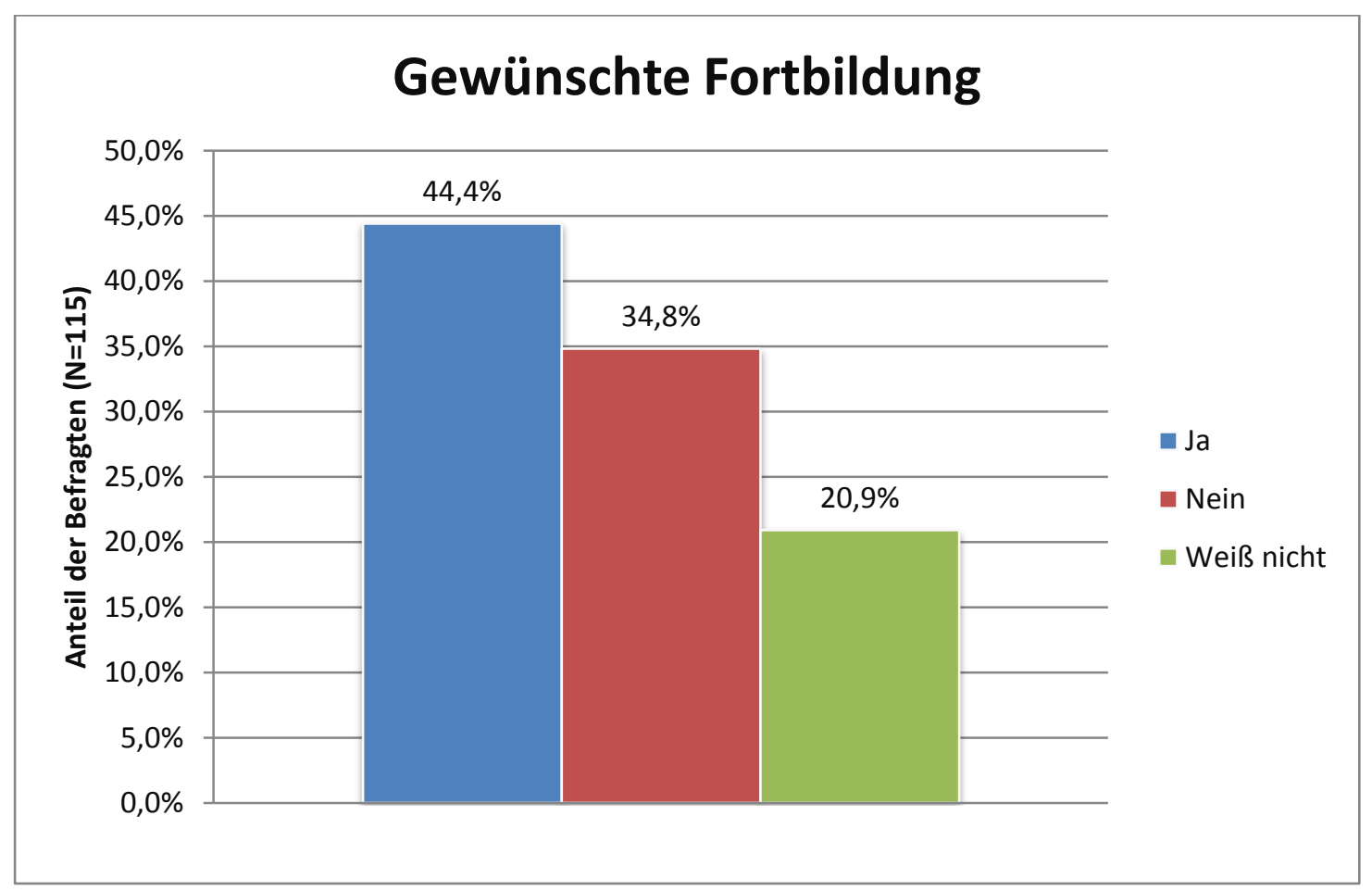




\subsection{Finanzierung von Beratung}

Die Fragen 6.1 bis 6.3 bezogen sich auf die Finanzierung von ärztlicher Beratung zur PV.

\subsubsection{Wurde Beratung zur Patientenverfügung schon abgerechnet?}

Die große Mehrheit der befragten Ärzte, die zum Thema PV beraten, rechnete bislang kein Beratungsgespräch ab (84,6\%). Einige rechneten die Gespräche über Beratungsziffern der GOÄ und der GKV ab (je 5,1\%). Vier Ärzte gaben an, die Beratung als Individuelle Gesundheitsleistung (IGeL) abzurechnen (3,4\%). Sonstige Möglichkeiten, wie z.B. Kaffeekasse oder pauschal fünf Euro, wurden vereinzelt genannt (5,1 \%). (Abb. 19)

Abbildung 19: Wie Beratungsgespräche bisher abgerechnet wurden.

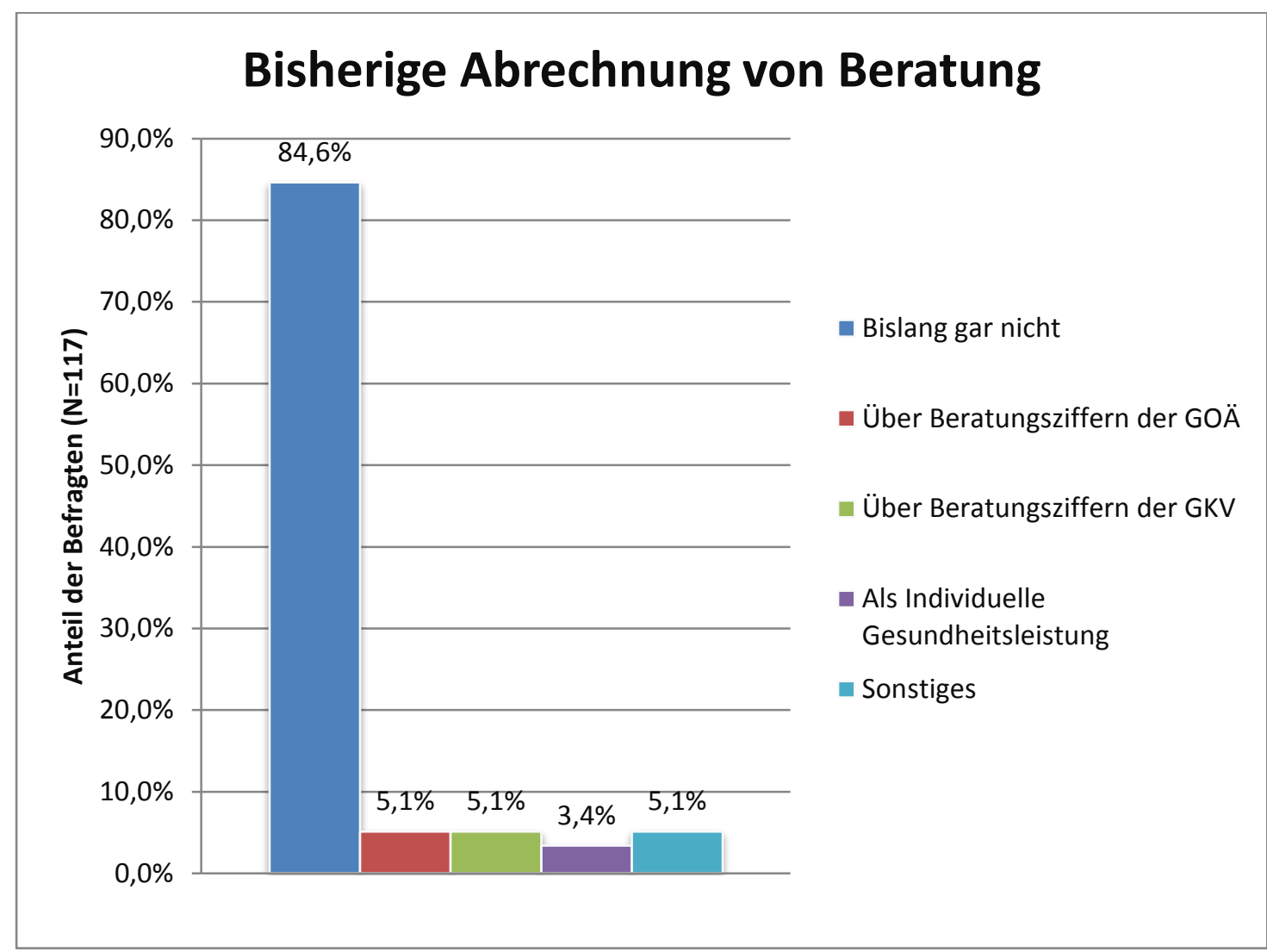




\subsubsection{Sind die Abrechnungsmöglichkeiten ausreichend?}

Die Mehrheit der Befragten hielt die bestehenden Abrechnungsmöglichkeiten für nicht ausreichend (62,5\%), nur etwa ein Achtel tat dies (14,8\%). Es fiel auf, dass 29 Studienteilnehmer keine Angaben machten (24,8\% der Befragten).

Es konnte kein signifikanter Zusammenhang zwischen dem Meinungsbild zu den Abrechnungsmöglichkeiten und der Tatsache, ob zu PV beraten wird oder nicht, festgestellt werden. Unabhängig davon, ob die Ärzte beraten, vertreten sie mehrheitlich die Ansicht, dass die Abrechnungsmöglichkeiten für Beratung zu PVen nicht ausreichen. (Abb. 20)

Abbildung 20: Stellungnahme zu der Aussage: „, Die bestehenden Möglichkeiten zur Abrechnung von Beratungsgesprächen sind ausreichend."

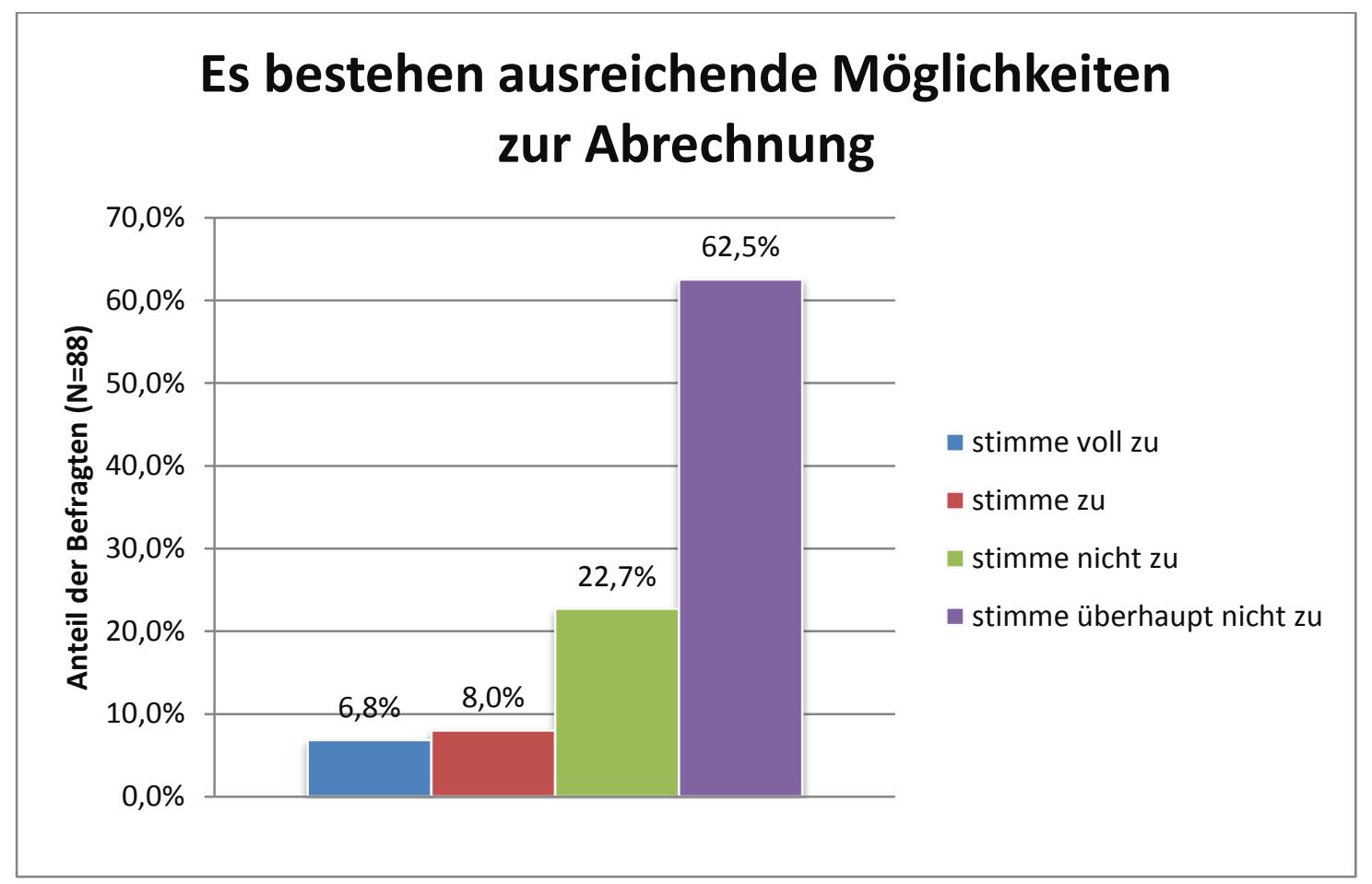




\subsubsection{Wie sollte ärztliche Beratung finanziert werden?}

Über die Hälfte der Befragten sprach sich dafür aus, dass die Beratung zur PV eine von der Solidargemeinschaft finanzierte Leistung sein sollte (56,3\%); ein Drittel sah den Patienten in der Pflicht (35,7 \%). Neun Teilnehmer kreuzten „Sonstiges“ (z.B. anteilige Finanzierung durch Krankenkasse und Patient) an (8\%). Fünf Befragte machten keine Angaben.

Zwischen dem Antwortverhalten der Berater und der Nicht-Berater konnte kein signifikanter Unterschied festgestellt werden. (Abb. 21)

Abbildung 21: Was sollte ärztliche Beratung zur PV sein?

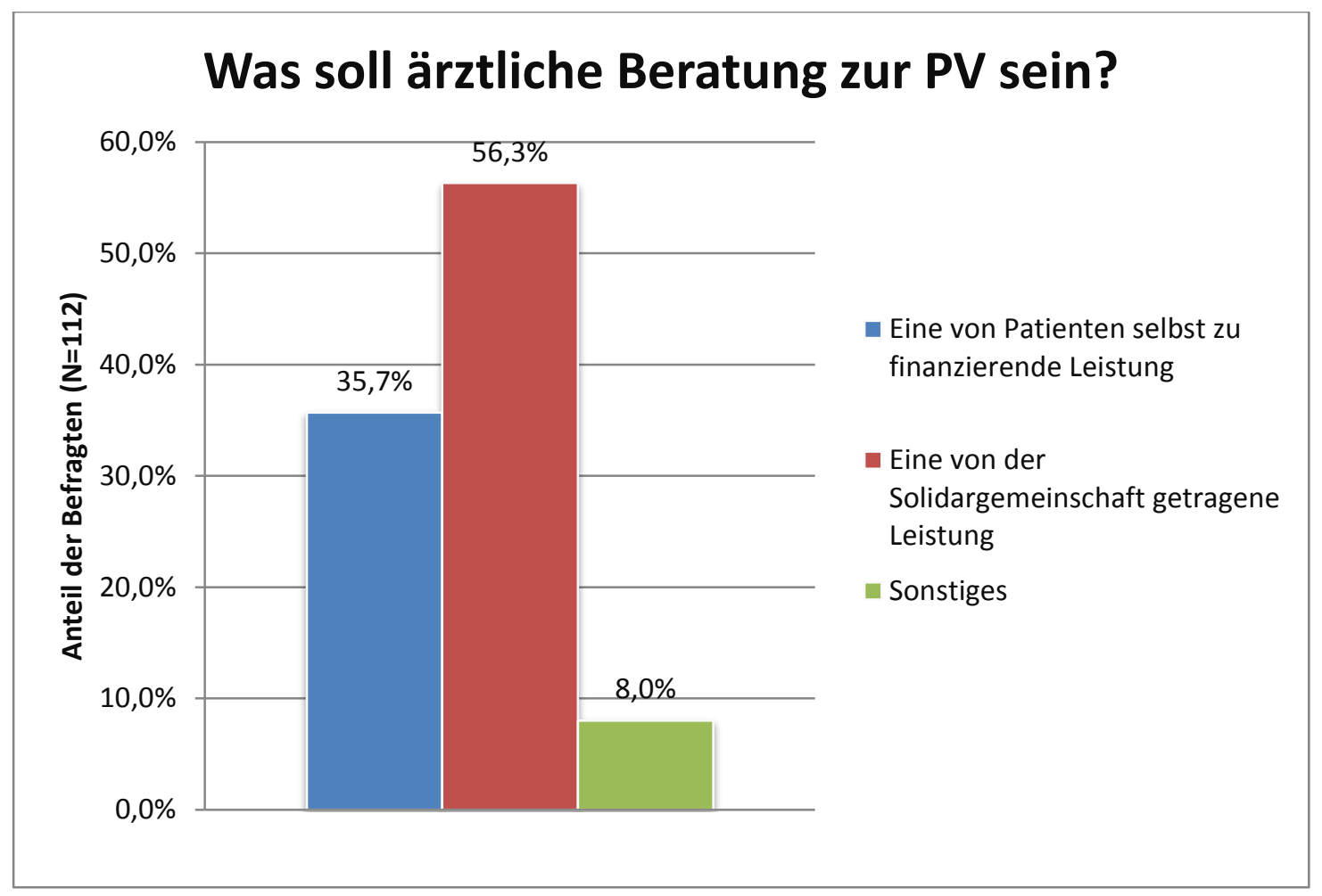




\subsection{Weitere Anmerkungen, Hinweise und Kommentare}

Am Ende des Fragebogens war Platz für weitere Hinweise, Anmerkungen und Kommentare, der von elf Ärzten genutzt wurde. Von drei Ärzten wurde nochmal die aktuelle Abrechnungssituation angesprochen. Sie sprachen sich dafür aus, dass ein Beratungsgespräch eine von der Solidargemeinschaft getragene Leistung sein und es eine Abrechnungsziffer der Gesetzlichen Krankenversicherungen geben sollte. Einer betonte sogar, dass die Beratung zur PV „ohne Vergütung keine weitere Aufgabe im Bereich der GOÄ“ sei. Ein anderer Teilnehmer der Studie forderte mehr Aufklärung über PVen durch die Medien. Auch wurde von drei Ärzten betont, dass eine Auseinandersetzung mit dem Thema PV bzw. die rechtzeitige Beratung zu verständlichen Formulierungen und den möglichen Behandlungssituationen einen höheren Stellenwert einnehmen oder sogar verpflichtend sein sollte. Häufig gäbe es keine Möglichkeit, sich dafür Zeit zu nehmen. Es wurde vorgeschlagen, das Thema PV und VV regulär in die Aufklärungsgespräche vor größeren Eingriffen mit einzubeziehen. Zwei Ärzte betonten das Thema VV. Dieses sollte „breiter diskutiert“ werden und an eine PV „gekoppelt“ werden. Ein Teilnehmer der Studie warnte davor, dass „Angehörige und Pflegepersonal bisweilen dazu neigen würden, schon allein das Vorliegen einer Vollmacht, ohne eingehende Prüfung der eigentlichen Inhalte, als eine Verpflichtung zur Therapieunterlassung anzusehen“. Schließlich wies ein Arzt darauf hin, dass in der Frage 5.1 nicht ausreichend zwischen allgemeiner und fachlicher Beratung zur PV unterschieden wurde. 


\subsection{Tabellarische Übersicht über die Signifikanzen}

Eine Kernfrage der Studie war, welche Ärzte zum Thema PV beraten. Deswegen wurde die Frage, ob die teilnehmenden Ärzte zum Thema PV beraten oder nicht, auf Signifikanzen in Bezug auf andere Fragen dieser Studie getestet. Die Tabelle 3 fasst die gefundenen Signifikanzen zusammen.

Tabelle 3: Signifikanzen in Bezug auf die Tatsache, ob die befragten Ärzte zum Thema PV beraten oder nicht.

\begin{tabular}{|c|c|c|c|}
\hline & Beratung: Ja $(n=85)$ & Beratung: Nein $(n=31)$ & $\mathbf{p}$ \\
\hline $\begin{array}{l}\text { Geschlecht: } \\
\text { Männlich } \\
\text { Weiblich }\end{array}$ & $\begin{array}{l}53(81,5 \%) \\
32(62,7 \%)\end{array}$ & $\begin{array}{l}12(18,5 \%) \\
19(37,3 \%)\end{array}$ & 0,023 \\
\hline $\begin{array}{l}\text { Facharztausbildung: } \\
\text { Allgemeinmedizin } \\
\text { Anästhesie }\end{array}$ & $\begin{array}{l}45(91,8 \%) \\
12(50 \%)\end{array}$ & $\begin{array}{l}4 \quad(8,2 \%) \\
12(50 \%)\end{array}$ & $\begin{array}{l}0,001 \\
0,044\end{array}$ \\
\hline $\begin{array}{l}\text { Tätigkeitsbereich: } \\
\text { Krankenhaus } \\
\text { Praxis }\end{array}$ & $\begin{array}{l}33(61,1 \%) \\
54(88,5 \%)\end{array}$ & $\begin{array}{ll}21 & (38,9 \%) \\
7 & (11,5 \%)\end{array}$ & $\begin{array}{l}0,018 \\
0,0008\end{array}$ \\
\hline $\begin{array}{l}\text { Selbst eine PV erstellt: } \\
\text { Ja } \\
\text { Nein }\end{array}$ & $\begin{array}{l}28(87,5 \%) \\
56(67,5 \%)\end{array}$ & $\begin{array}{l}4 \quad(12,5 \%) \\
27(32,5 \%)\end{array}$ & 0,03 \\
\hline $\begin{array}{l}\text { Frequenz des Ansprechens: } \\
\text { Täglich } \\
\text { Wöchentlich } \\
\text { Monatlich } \\
\text { Seltener }\end{array}$ & $\begin{array}{l}4(100 \%) \\
30(85,7 \%) \\
34(79,1 \%) \\
16(50 \%)\end{array}$ & $\begin{array}{lc} & -- \\
5 & (14,3 \%) \\
9 & (20,9 \%) \\
16 & (50 \%)\end{array}$ & 0,0008 \\
\hline $\begin{array}{l}\text { Frequenzsteigerung: } \\
\text { Ja } \\
\text { Nein }\end{array}$ & $\begin{array}{l}37(86,1 \%) \\
48(69,6 \%)\end{array}$ & $\begin{array}{ll}6 & (13,9 \%) \\
21 & (30,4 \%)\end{array}$ & 0,049 \\
\hline $\begin{array}{l}\text { Verbindliche Beratung?: } \\
\text { Stimme voll zu } \\
\text { Stimme zu }\end{array}$ & $\begin{array}{l}33(94,3 \%) \\
20(60,6 \%)\end{array}$ & $\begin{array}{l}2(5,7 \%) \\
13(39,4 \%)\end{array}$ & 0,011 \\
\hline
\end{tabular}


Ergebnisse

\begin{tabular}{|l|l|ll|l|}
\hline Stimme nicht zu & $16(66,7 \%)$ & 8 & $(33,3 \%)$ \\
Stimme überhaupt nicht zu & $14(63,6 \%)$ & $(36,3 \%)$ & \\
\hline Beratungskompetent?: & $32(82,1 \%)$ & $7 \quad(17,9 \%)$ & 0,02 \\
Stimme voll zu & $45(76,3 \%)$ & $14(23,7 \%)$ & \\
Stimme zu & $7 \quad(50 \%)$ & $7 \quad(50 \%)$ & \\
Stimme nicht zu & $1 \quad(25 \%)$ & $3 \quad(75 \%)$ & \\
Stimme überhaupt nicht zu & 14 & \\
\hline
\end{tabular}




\section{Diskussion}

Ziel der Studie war es herauszufinden, in welchem Ausmaß und in welcher Form ärztliche Beratung zum Thema PV stattfindet und welche Qualität diese Beratung hat. In der am 1. September 2009 in Kraft getretenen Gesetzesänderung wird eine ärztliche Beratung zur PV zwar nicht als Wirksamkeitsvoraussetzung gefordert, in der Gesetzesbegründung aber sehr wohl empfohlen (Stünker et al. 2008). Auch die BÄK und die Zentrale Ethikkommission empfehlen eine ärztliche Beratung vor Erstellung einer PV (BÄK 2010).

Befragt wurden Ärztinnen und Ärzte, die an einer Weiterbildung zum Thema Palliativmedizin teilnahmen. Die Befragten kamen aus unterschiedlichen Fachrichtungen und waren sowohl im niedergelassenen Bereich als auch in Krankenhäusern tätig. Es konnte angenommen werden, dass aufgrund des Umgangs mit schwerstkranken und sterbenden Patienten ein großes Interesse am Thema bestand. Die Rücklaufquote von $100 \%$ bestätigte diese Annahme.

In den folgenden Abschnitten sollen die Studienergebnisse diskutiert und mögliche Konsequenzen für die Debatte über den Umgang mit ärztlicher Beratung zum Thema PV aufgezeigt werden.

\subsection{Wer berät zum Thema Patientenverfügung?}

Eine große Mehrheit der befragten Ärzte (73,3 \%) gab an, zum Thema PV zu beraten, wobei männliche Ärzte signifikant häufiger Beratungsgespräche führten als ihre weiblichen Kolleginnen. Alter und Tätigkeitsdauer hatten keinen Einfluss auf die Häufigkeit der Beratung.

Die Fachrichtung, in der am häufigsten zum Thema PV beraten wurde, war die Allgemeinmedizin. Dies dürfte damit zusammen hängen, dass der Allgemeinmediziner in Deutschland entsprechend dem Modell zur Hausarztzentrierten Versorgung (vgl. SGB V $\S 73 b)$ die erste Anlaufstelle für medizinische Fragen ist. Dies führt dazu, dass in der niedergelassenen Praxis öfter ein Beratungsgespräch stattfindet als im Krankenhaus. Auch ist die durchschnittliche Dauer des Patientengesprächs in der niedergelassenen Praxis länger als im Krankenhaus. So hat der niedergelassene Arzt mehr Möglichkeiten, Gespräche mit dem 
Patienten zu führen, die über die akute medizinische Situation hinausgehen (VgI. Jährig und Koch 1982, Deveugele et al. 2002).

Am seltensten wurde in der Anästhesiologie beraten. Dies lässt sich erklären, wenn man sich die Einsatzgebiete von Anästhesisten vor Augen führt, in denen es sich häufig um die Notfallversorgung oder die Anästhesie handelt, in denen oft nur ein zeitlich kurzer Patientenkontakt vorkommt.

Die Ergebnisse zeigen, dass ein Unterschied in der Beratungshäufigkeit besteht zwischen Ärzten, die im Krankenhaus arbeiten, und niedergelassenen Ärzten. Im Krankenhaus wird deutlich weniger zum Thema PV beraten, als im niedergelassenen Bereich. Dies korrespondiert mit dem oben genannten Ergebnis, dass Allgemeinmediziner insgesamt signifikant häufiger beraten als Ärzte anderer Fachrichtungen. Die Regelung der Hausarztzentrierten Versorgung in Deutschland, die mit dem GKV-WettbewerbsStärkungsgesetz (WSG) am 1. April 2007 in Kraft getreten ist (GKV-WSG 2007), hat zum Ziel, dass der Hausarzt die Steuerung der ambulanten Versorgung übernimmt. Er soll für die Patienten eine Anlaufstelle sein, die ihm bei der Vermittlung an die verschiedenen Institutionen im Gesundheitswesen hilft und den weiteren Verlauf koordiniert und organisiert. Die Tatsache, dass der Allgemeinmediziner für die Patienten am ehesten der Ansprechpartner in Bezug auf die Beratung zur PV zu sein scheint, zeigt, dass dieses Modell in diesem Rahmen seine Anwendung findet. Des Weiteren ist die hausärztliche Fürsorge am ehesten für eine ausführliche Beratung geeignet. Häufig hat der Arzt seinen Patienten schon über einen längeren Zeitraum betreut und kann ihn so in Bezug auf seine Wünsche, sollte er einwilligungsunfähig werden, suffizienter beraten. Der Patient hat die Möglichkeit in der Häuslichkeit in Ruhe über die Entscheidungen nachzudenken, die er in einer PV formuliert. Im Rahmen eines Krankenhausaufenthalts ist dieses meist nicht möglich. Das Personal wechselt häufig und die immer kürzer werdenden Liegezeiten lassen ein ausführliches Beratungsgespräch eventuell gar nicht zu.

So lässt sich gut nachvollziehen, dass Beratungsgespräche zur PV signifikant häufiger beim Allgemeinmediziner stattfinden.

Die Mehrheit der Ärzte gab an, ihre Patienten auf die Möglichkeit einer PV hinzuweisen. Dies geschah im niedergelassenen Bereich genauso wie im Krankenhaus. Die meisten Ärzte, die eine PV grundsätzlich empfehlen, sprechen ihre Patienten auch auf das Thema an (81,7 \%). 
Dies weist auf eine hohe Akzeptanz des Instruments der PV hin. Es scheint, dass die befragten Ärzte die PV in ihren ärztlichen Alltag mit aufgenommen haben und damit das Ziel, die nachhaltige Stärkung der Patientenautonomie, erreicht werden kann.

Die große Mehrheit der befragten Ärzte empfiehlt ihren Patienten die Erstellung einer PV (91,3 \%). Dies unterstreicht die Wichtigkeit und Akzeptanz dieses Instruments aus Sicht der Ärzte. Die Empfehlung zur Erstellung einer PV wird unabhängig vom Alter, der Fachrichtung, dem Tätigkeitsbereich und der Tätigkeitsdauer ausgesprochen. Auch der Umstand, ob der Arzt für sich selbst eine PV erstellt hat, hat keinen Einfluss darauf.

Hingegen fällt auf, dass männliche Ärzte signifikant häufiger die Erstellung einer PV empfehlen als Ärztinnen. Dies korrespondiert mit dem Ergebnis zur Beratungshäufigkeit. Es scheint, dass sich die männlichen Ärzte in einer anderen Form mit dem Thema PV auseinandersetzen als ihre weiblichen Kollegen. Auch in einer anderen Studie trat die Gruppe der männlichen Ärzte hervor. In dieser wurden die Teilnehmer nach einer Befragung je nach ihrer Einstellung zum Thema PV in drei verschiedene Gruppen eingeteilt. Die männlichen Ärzte lehnten am häufigsten die Gruppe ab, in denen eine gültige PV an eine notarielle Beglaubigung gebunden sein müsse. Sie favorisierten offensichtlich die anderen Gruppen, in denen eine PV als eine gültige Willensäußerung beschrieben wurde oder als wichtiges Mittel zur Kommunikation zwischen Arzt und Patient. Auch hier könnte dies damit verbunden sein, dass gerade die männlichen Ärzte nicht wollen, dass ihnen eine medizinische Entscheidung letztlich abgenommen und eine richterliche Entscheidung daraus gemacht würde. Man könnte vermuten, dass die männlichen Ärzte dies eher als einen Konkurrenzkampf mit den Juristen sehen und deswegen eine PV, die mit ihrer Beratung erstellt wurde, am ehesten als eine Art Vorabentscheidung oder Selbstbestimmung sehen. Für die weiblichen Ärzte scheint dieser Konkurrenzkampf nicht gleichermaßen relevant zu sein. Man könnte vermuten, dass Frauen in ihrer beziehungsethischen Sichtweise größeren Wert auf Gespräche mit Angehörigen legen und sich in der Entscheidungsfindung vorab nicht in den Vordergrund stellen müssen (Oorschot und Simon 2006).

Auch scheint relevant zu sein, ob die Ärzte für sich selbst eine PV erstellt haben oder nicht. Haben Ärzte selbst eine PV, so ist die Wahrscheinlichkeit, dass sie zu diesem Thema beraten, sehr viel höher. Dies könnte man darauf zurückführen, dass sich diese Ärzte schon eingehend mit diesem Thema beschäftigt haben und ihnen auch die Formalitäten einer PV bewusst sind. Überdies kann man bei Ärzten, die für sich selbst eine PV einsetzen, auch 
davon ausgehen, dass die Akzeptanz dieses Instruments höher ist, als bei Ärzten, die dies nicht für sich einsetzen.

\subsection{Fragen zur Beratungsqualität}

Über 90 \% der befragten Ärzte gaben an, sich zum Thema PV gut informiert zu fühlen. Die hohe Zahl könnte darauf zurückzuführen sein, dass diese Studie mit Ärzten durchgeführt wurde, die an einer Weiterbildung zur Palliativmedizin teilnehmen. Es ist anzunehmen, dass Ärzte, die mit schwerstkranken und sterbenden Patienten zu tun haben, bereits mit dem Thema konfrontiert wurden. Die befragten Ärzte konnten jedoch lediglich zu ihrer subjektiven Einschätzung befragt werden. Möglich ist also, dass die Studienteilnehmer lediglich meinen, gut zu diesem Thema informiert zu sein, oder auch nicht zugeben wollen, dass sie es nicht sind. Wenig überraschend ist, dass sich die Ärzte, die Patienten zu diesem Thema beraten, insgesamt besser informiert fühlen, als die Ärzte, die nicht beraten. Von den Beratern fühlt sich auch die insgesamt größere Mehrheit (über 95 \%) gut informiert. Auch in Bezug auf die Einschätzung der Beratungskompetenz fühlten sich die beratenden Ärzte insgesamt kompetenter. Dabei könnte es sich durchaus um eine in der Praxis erworbene Kompetenz handeln, die sich im Verlauf zurückliegender Beratungsgespräche und den damit verbundenen Erfahrungen entwickelt hat. Trotzdem fühlt sich auch die Mehrheit der nicht beratenden Ärzte kompetent genug, zum Thema PV zu beraten. Auch dies könnte mit der Auswahl der Studienteilnehmer zusammenhängen.

Die Studie ergab, dass ein Beratungsgespräch durchschnittlich etwas mehr als 20 Minuten dauert und zwar unabhängig davon, ob es in der niedergelassenen Praxis oder im Krankenhaus durchgeführt wird. Dies bedeutet, dass für die Beratung zur PV deutlich mehr Zeit aufgewandt wird, als für die Visite im Krankenhaus (3-4 Minuten pro Patient, vgl. Jährig und Koch 1982) oder das normale Patientengespräch beim niedergelassenen Arzt (8 Minuten pro Patient, vgl. Deveugele et al. 2002). Angesichts der Komplexität des zu beratenden Themas erscheint die Zeit aber immer noch relativ kurz.

Fortbildung zum Thema PV wurde - unabhängig davon, ob zum Thema PV beraten wurde oder nicht - von knapp der Hälfte der Ärzte (44,4\%) gewünscht. Ein Drittel der Ärzte $(34,8 \%)$ sah keinen Fortbildungsbedarf. Angesichts der Tatsache, dass sich die meisten Ärzte als „gut informiert“ und „kompetent“ in Bezug auf die Beratung zur PV eingeschätzt hatte, überrascht es, dass die Mehrheit der Ärzte dennoch einen Fortbildungsbedarf sah. 
Die Ärzte hatten die Möglichkeit, ihren Fortbildungswunsch inhaltlich zu konkretisieren, was nur von einem Teil wahrgenommen wurde. Am häufigsten wurden Fortbildungen zu den Rechtsgrundlagen gewünscht. Dieser Bedarf an rechtlichen Informationen korrespondiert mit dem Ergebnis anderer Studien. So wurde 2006 in einer Umfrage festgestellt, dass es bei befragten Ärzten große Unsicherheiten in Bezug auf die Grenzen zwischen erlaubter passiver bzw. indirekter und nicht erlaubter aktiver Sterbehilfe gab (Oorschot und Simon 2006). Auch in einer fünf Jahre älteren Studie wurden erhebliche rechtliche Unsicherheiten bei den Ärzten in Bezug auf Entscheidungen am Lebensende festgestellt (Weber et al. 2001).

\subsection{Fragen zum Stellenwert der ärztlichen Beratung}

Die Bedeutung und mögliche Verbindlichkeit einer ärztlichen Beratung zur PV wurde im Vorfeld des neuen Gesetzes kontrovers diskutiert. Auch die Teilnehmer der Studie waren sich hinsichtlich einer verbindlichen Beratung als Gültigkeitsvoraussetzung für eine PV keineswegs einig. Dies wurde auch davon beeinflusst, ob sie selbst zu den Ärzten gehören, die zu diesem Thema Patienten beraten, oder nicht. Insgesamt stimmten über die Hälfte der Teilnehmer (60\%) einer Verbindlichkeit zu. Aber auch von den Ärzten, die nicht zum Thema PV beraten, sprach sich fast die Hälfte $(48,4 \%)$ für eine Verbindlichkeit von ärztlicher Beratung zur PV als Gültigkeitsvoraussetzung aus, wie sie auch in dem Gesetzentwurf von Bosbach vorgesehen war.

Des Weiteren sieht die Mehrheit der befragten Ärzte (51,7 \%) eine ärztliche Beratung als wichtige Voraussetzung, damit eine PV für den später damit konfrontierten Arzt eine Hilfestellung sein kann. Der betreffenden Aussage stimmten fast ein Viertel der Ärzte $(24,1$ \%) voll zu.

Aus welchen Gründen wird die ärztliche Beratung zur PV von den befragten Ärzten als so wichtig eingestuft? Die Ergebnisse der Studie geben verschiedene Erklärungsansätze.

So stimmten 85 \% der Ärzte der Aussage zu, dass ärztliche Beratung für den zukünftigen Patienten wichtig sei, um die in einer PV zu beschreibenden Situationen zu verstehen. 84 \% hielten die Beratung für das Verständnis des Patienten von Schaden und Nutzen ärztlicher Maßnahmen für wichtig. Beide Aussagen wurden von Beratern und Nicht-Beratern gleich bewertet. Dies spiegelt sich auch in den Empfehlungen der BÄK wider (BÄK 2010). Eine Studie, in der Patienten, gesunde Personen, Ärzte und Pflegende befragt wurden, belegt diesen Eindruck. Die Mehrheit der Befragten zeigten große Unsicherheiten überhaupt 
Behandlungssituationen im Voraus festzulegen und es wurde dargelegt, dass Unkenntnis über verschiedene Maßnahmen Entscheidungen beeinflusst haben (Sahm 2006).

Ein weiterer wichtiger Grund, der für eine ärztliche Beratung zur PV spricht, ist das Problem, dass der Wortlaut einer PV, die ohne ärztliche Beratung erstellt wurde, eventuell nicht mit dem tatsächlichen Willen des Patienten übereinstimmt. Dieser Aussage stimmten 78 \% der befragten Ärzte zu, wobei Ärzte, die zum Thema PV beraten, signifikant häufiger dieser Aussage zustimmten. Auch auf dieses Problem weist die BÄK und die Zentrale Ethikkommission hin (BÄK 2010). Auch empirische Studien zeigen, dass dieses Problem in der Praxis besteht. Eine Studie mit halbstrukturierten Interviews aus Bonn zeigt, dass der im Interview geäußerte Wille durchaus häufig nicht mit der erstellten PV übereinstimmt. So wollten die Befragten beispielsweise im Interview medizinische Maßnahmen für sich in Anspruch nehmen, die sie in ihrer PV abgelehnt hatten (Becker et al. 2009). Auch eine Befragung, die die Deutsche Hospizstiftung 2005 durchführte, ergab, dass gerade ältere Menschen wussten, was sie verfügen wollten, es aber Unsicherheiten über Form und Formulierung gab (Deutsche Hospizstiftung 2005).

\subsection{Fragen zur Finanzierung von ärztlicher Beratung}

Die Studie untersuchte auch die Fragen, wie eine ärztliche Beratung zur PV finanziert wird bzw. finanziert werden sollte. Es ist ziemlich beeindruckend, dass die große Mehrheit der Ärzte, die Beratungsgespräche zum Thema PV führen, den damit verbundenen Zeitaufwand bislang nicht abrechneten (84,5 \%). Nur wenige gaben an, die Beratungsziffern der Gebührenordnung für Ärzte oder der Gesetzlichen Krankenversicherung zu nutzen (je 7,1%). Im später vom Bundestag beschlossenen Gesetzesentwurf von Stünker wurde die ärztliche Beratung zur PV als eine vom Patienten selbst zu finanzierende Leistung beschrieben (Stünker 2008). Dies bedeutet, dass die beratenden Ärzte ein Beratungsgespräch als eine Individuelle Gesundheitsleistung (IGeL) in Rechnung zu stellen haben. Dies taten allerdings nur vier der in dieser Studie befragten Ärzte und somit weniger als $5 \%$ der Ärzte, die Beratungsgespräche zum Thema PV führten. Eventuell hatten die Ärzte moralische Bedenken, ihren Patienten die entsprechenden Kosten in Rechnung zu stellen. Offensichtlich halten diese Ärzte die ärztliche Beratung zur PV aber trotzdem für so wichtig, dass sie sie unbezahlt durchführen. Im Gesetzesentwurf von Bosbach wurde die Beratung als eine von der Solidargemeinschaft getragene Finanzierung der Beratung gesehen (Bosbach 2008). Eine 
solche Lösung favorisierte auch die Mehrheit der an der Studie teilnehmenden Ärzte (56,3 \%) - und zwar unabhängig davon, ob sie selbst Beratungsgespräche durchführten oder nicht.

Dies erfordert durchaus Handlungsbedarf vom Gesetzgeber, der die Umstände der Abrechnung nicht abschließend geklärt hat. Dies spiegelt sich auch im allgemeinen Meinungsbild dieser Studie wider. Nur knapp $15 \%$ der Teilnehmer halten die bestehenden Möglichkeiten zur Abrechnung für Beratungsgespräche für ausreichend. Die Mehrheit $(62,5$ \%) stimmte der betreffenden Aussage überhaupt nicht zu.

Abschließend könnte man auch überlegen, ob sich die Erstellung einer konkreten und eindeutigen PV mit ärztlicher Hilfe nicht auch positiv auf die Kosten für die Solidargemeinschaft auswirken könnte. Im Zweifel über den mutmaßlichen Willen des Patienten ist der Arzt dazu angehalten, die Therapiemöglichkeiten voll auszuschöpfen. Gerade in der terminalen Phase bedeutet dies häufig auch eine intensivmedizinische Behandlung. In den Veröffentlichungen der Krankenkassen wird deutlich, dass in den letzten Lebensmonaten die Gesundheitsausgaben für die Versicherten stark ansteigen (Wissenschaftliches Institut der PKV 2006). Liegt eine präzise PV vor, so kann in einigen Fällen auf vielleicht teure Maßnahmen verzichtet werden, weil der Patient diese eindeutig nicht mehr wünscht.

Da im Rahmen dieser Studie Fachärzte befragt wurden, die an einer palliativmedizinischen Weiterbildung teilgenommen haben, sind die Interpretationsmöglichkeiten dieser Studie limitiert. Ein gewisses Interesse an dieser Thematik bei den Befragten war somit vorausgesetzt und es ist möglich, dass der Anteil der Ärzte, die den Umgang mit PVen in ihren Alltag aufgenommen und die PV als Instrument akzeptiert haben, in dieser Studie größer ist als in der Gesamtheit der Ärzte in Deutschland. Da die Ergebnisse dieser Studie jedoch zeigen, dass die PV im Allgemeinen und die ärztliche Beratung zur PV im Speziellen bei einer großen Mehrheit der Befragten auf deutlich positive Resonanz stößt, könnte man annehmen, dass auch bezogen auf die Ärzte insgesamt ein positiver Trend in Bezug auf die Akzeptanz der PV besteht. In der vorliegenden Studie wurden zudem Ärzte aus völlig verschiedenen Fachbereichen befragt. Dies zeigt auch, dass fachübergreifend ein Interesse an diesem Instrument besteht. 


\section{Zusammenfassung und Ausblick}

Die Ergebnisse dieser Arbeit zeigen deutlich, dass der Umgang mit ärztlicher Beratung zum Thema PV noch längst nicht abschließend geklärt ist. Das am 1. September 2009 in Kraft getretene Dritte Gesetz zur Änderung des Betreuungsrechts stärkt das Recht des Patienten auf Selbstbestimmung. Es lässt jedoch viele Fragen im Zusammenhang mit der ärztlichen Beratung zur PV, die eine wichtige Voraussetzung für die antizipierende Wahrnehmung des Selbstbestimmungsrechts seitens des Patienten darstellt, offen.

Ziel der vorliegenden Studie war es herauszufinden, ob und in welchem Rahmen ärztliche Beratung zur PV stattfindet, und wo es Probleme im Zusammenhang mit dieser Beratung gibt. Hierzu wurden Ärztinnen und Ärzte befragt, die an einer Weiterbildung zur Palliativmedizin teilnahmen.

Die Beteiligung von $100 \%$ zeigt, dass ärztlicherseits ein großes Interesse an dem Thema PV besteht.

Die Mehrheit der befragten Ärzte gab ferner an, zum Thema PV zu beraten, wobei vor allem Allgemeinmediziner in der niedergelassenen Praxis ihre Patienten über die Möglichkeiten einer PV aufklären. Dies kann auf die Regelung der ,Hausarztzentrierten Versorgung' in Deutschland zurückgeführt werden. Aber auch in der stationären Versorgung und in anderen Fachrichtungen werden PVen gegenüber den Patienten thematisiert. Die große Mehrheit der befragten Ärzte empfiehlt die Erstellung einer PV, was für eine große Akzeptanz und Wertschätzung des Instruments spricht. Die Mehrheit der Befragten gab auch an, ihre Patienten von sich aus auf die Möglichkeit einer PV anzusprechen. Dies legt die Schlussfolgerung nahe, dass die PV im ärztlichen Alltag ihren Platz gefunden hat, und die Ärzteschaft den Wunsch nach Selbstbestimmung und die Umsetzung des mutmaßlichen Patientenwillens ernst nimmt.

Die Ärzte sind nach eigener Einschätzung für die Beratung gut vorbereitet. Die befragten Ärzte fühlten sich gut informiert und schätzen sich als ausreichend kompetent ein, um Patienten zum Thema PV zu beraten. Zu berücksichtigen ist allerdings, dass das Kollektiv der Befragten wahrscheinlich besser zu diesem Thema informiert ist, als der Durchschnitt der Ärzte in Deutschland. Aber auch dieses Kollektiv wünscht sich mehrheitlich Fortbildungen. Wie genau ein Beratungsgespräch aufgebaut sein könnte, und welche rechtlichen Aspekte 
man bei der Beratung besonders beachten muss, kann man den öffentlichen Medien nicht entnehmen. Ferner wäre es wünschenswert, Empfehlungen oder Standards zu erarbeiten, die den beratenden Ärzten helfen, ein Beratungsgespräch sinnvoll zu gestalten. Ärztliche Beratung wird zwar von der BÄK und der Zentralen Ethikkommission empfohlen, Kriterien für die Qualität ärztlicher Beratung zur PV werden jedoch nicht genannt.

Die Erarbeitung und Vorgabe von Standards, die die Beratung zur Patientenverfügung erleichtert und eine Qualitätssicherung des Beratungsgesprächs ermöglicht, sollte dringend eingeführt werden.

Der Stellenwert der ärztlichen Beratung aus Sicht der befragten Ärzte ist sehr hoch. Über die Hälfte der Befragten sprach sich für eine verbindliche Beratung zur PV aus. Ärztliche Beratung zur PV ist aus Sicht der Ärzte aus verschiedenen Gründen sinnvoll:

1) Der zukünftige Patient muss die in einer PV zu beschreibenden Situationen angemessen verstehen.

2) Das Verständnis von Schaden und Nutzen ärztlicher Maßnahmen seitens des Patienten muss gegeben sein.

3) Der Wortlaut der verfassten PV muss mit dem Willen des Patienten übereinstimmen.

Ein ärztliches Beratungsgespräch kann helfen, diese Punkte zu verwirklichen.

Letztlich bleibt der Aspekt der Finanzierung der Beratungsgespräche. Die große Mehrheit der befragten Ärzte hatte keine für sie akzeptable Möglichkeit gefunden, ein Beratungsgespräch abzurechnen. Dabei ist diese mit einem erheblichen Zeitaufwand verbunden: Ein Beratungsgespräch dauerte im Durchschnitt über 20 Minuten.

Wenn - wie in zahlreichen Stellungnahmen empfohlen - eine ärztliche Beratung zur PV stattfinden soll, dann muss diese auch angemessen vergütet werden. Eine eigene Abrechnungsziffer wäre eine Erleichterung für die beratenden Ärzte. Zu klären ist ferner, wer die Kosten für ein Beratungsgespräch übernimmt. Die Mehrheit der befragten Ärzte sprach sich für eine von der Solidargemeinschaft getragene Finanzierung aus. Diese würde Patienten unabhängig von ihrem Einkommen ermöglichen, sich bei der Erstellung einer PV ärztlich beraten zu lassen, und könnte somit dazu beitragen, die Anzahl qualifizierter PVen in der Praxis zu erhöhen. Es sollte somit zeitnah eine einheitliche Abrechnungsziffer geschaffen 
werden und durch solidarisch finanzierte Beratungsgespräche jedem die Möglichkeit gegeben werden, sich zur Erstellung einer Patientenverfügung beraten zu lassen.

In Bezug auf die Voraussetzungen und die Qualität einer ärztlichen Beratung zur PV sowie der Vergütung der Beratungsgespräche besteht demnach noch erheblicher Diskussions- und Handlungsbedarf.

Die vorliegende Studie beleuchtet das Thema Beratung zu PV aus Sicht der Ärzte. Es wäre sinnvoll und wünschenswert, auch die Sicht der Patienten bzw. Bürger, die sich zum Thema PV beraten lassen wollen, zu erfahren. Dies sollte Gegenstand künftiger Studien sein. 


\section{Anhang}

\subsection{Fragebogen}

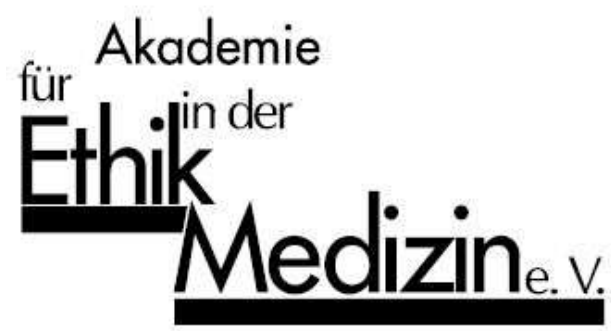

Georg-August-Universität, Humboldtallee 36, D-37073 Göttingen, E-Mail: info@aem-online.de, Internet: www.aem-online.de

Befragung „Beratung zur Patientenverfügung - eine ärztliche Aufgabe?“

Sehr geehrte Damen und Herren,

im Rahmen einer von PD Dr. Alfred Simon betreuten Doktorarbeit beschäftige ich mich mit dem Thema „Beratung zur Patientenverfügung - eine ärztliche Aufgabe?“.

Seit dem 1. September 2009 sind die Voraussetzungen für die Verbindlichkeit von Patientenverfügungen im Betreuungsrecht geregelt. Eine Beratung vor Erstellung einer Patientenverfügung ist vom Gesetz nicht vorgeschrieben, wird aber vom Gesetzgeber empfohlen.

In meiner Studie möchte ich untersuchen, welche Bedeutung Ärztinnen und Ärzte der ärztlichen Beratung zur Patientenverfügung beimessen, und welche Voraussetzungen ihrer Meinung nach gegeben sein müssen, damit sie Patienten angemessen beraten können.

Ich würde mich sehr über Ihre Beteiligung freuen!

Das Ausfüllen des Fragebogens dauert ca. 10 Minuten. Sollte der Platz für die Freitextantworten nicht ausreichen, können Sie die Rückseiten des Fragebogens verwenden.

Vielen Dank!

Ihre

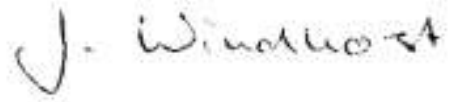

Julia Windhorst 
Befragung „Beratung zur Patientenverfügung - eine ärztliche Aufgabe?“

1. Angaben zur Person (Zutreffendes bitte ankreuzen)

\begin{tabular}{|c|c|}
\hline 1.1 & $\begin{array}{l}\text { Wie alt sind Sie? } \\
\square \leq 30 \text { Jahre } \square \text { 31-40 Jahre } \square \text { 41-50 Jahre } \square \text { 51-60 Jahre } \square>60 \text { Jahre }\end{array}$ \\
\hline 1.2 & $\begin{array}{l}\text { Ihr Geschlecht? } \\
\square \text { weiblich } \square \text { männlich }\end{array}$ \\
\hline 1.3 & $\begin{array}{l}\text { Welche Facharztausbildung haben Sie? } \\
\text { Ich bin Fachärztin/Facharzt für: }\end{array}$ \\
\hline 1.4 & $\begin{array}{l}\text { Wo sind Sie tätig? } \\
\square \text { Krankenhaus } \\
\square \text { Niedergelassener Bereich } \\
\square \text { Sonstiges, und zwar: }\end{array}$ \\
\hline 1.5 & $\begin{array}{l}\text { Wie lange sind Sie als Ärztin/Arzt tätig? } \\
\square \text { unter } 5 \text { Jahre } \square \text { 5-10 Jahre } \square \text { 11-20 Jahre } \square \text { über } 20 \text { Jahre }\end{array}$ \\
\hline 1.6 & $\begin{array}{l}\text { Haben Sie selbst eine Patientenverfügung für sich erstellt? } \\
\square J a \square \text { Nein }\end{array}$ \\
\hline
\end{tabular}

2. Aktueller Kenntnis- bzw. Informationsstand (Zutreffendes bitte ankreuzen)

2.1 Ich bin über das Thema Patientenverfügung gut informiert.
Ich stimme voll zu
Ich stimme überhaupt nicht zu 


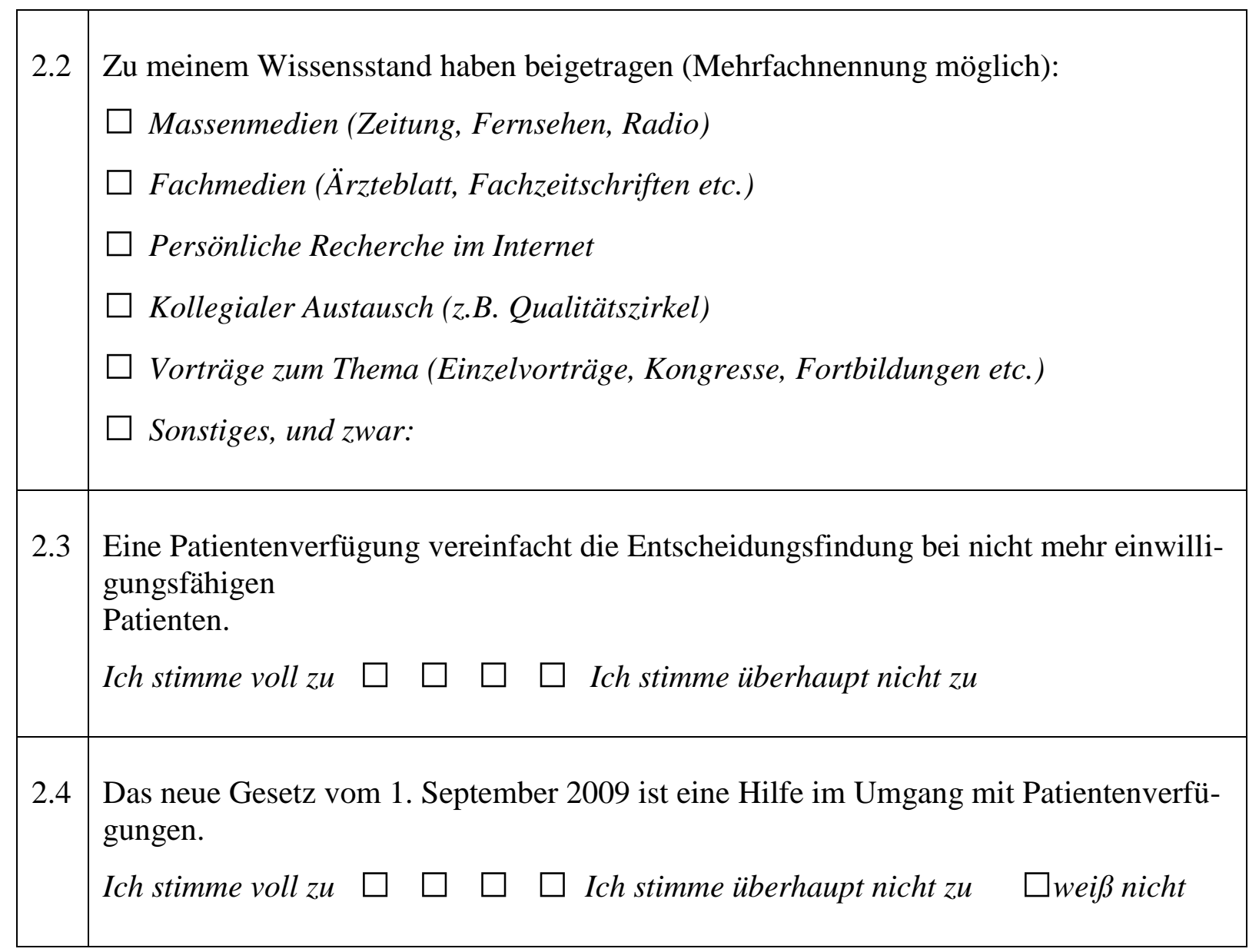

\section{Bisherige Beratungspraxis (Zutreffendes bitte ankreuzen)}

3.1 Wie häufig werden Sie von Patienten auf das Thema Patientenverfügung angesprochen?
täglich
$\square$ wöchentlich
monatlich
seltener
noch nie

3.2 Hat die gesetzliche Regelung der Patientenverfügung vom 1. September 2009 dazu geführt, dass Sie

nun häufiger von Patienten auf das Thema Patientenverfügung angesprochen werden?

Ja, etwas häufiger

Nein, die Häufigkeit hat sich nicht merklich verändert

Nein, die Häufigkeit hat sich eher verringert

3.3 Beraten Sie Patienten zum Thema Patientenverfügung?

Nein (weiter mit Frage 3.7) $\square J a$

3.4 Wie lange dauert eine solche Beratung im Durchschnitt?

Durchschnittlich ca. Minuten. (Bitte eintragen) 


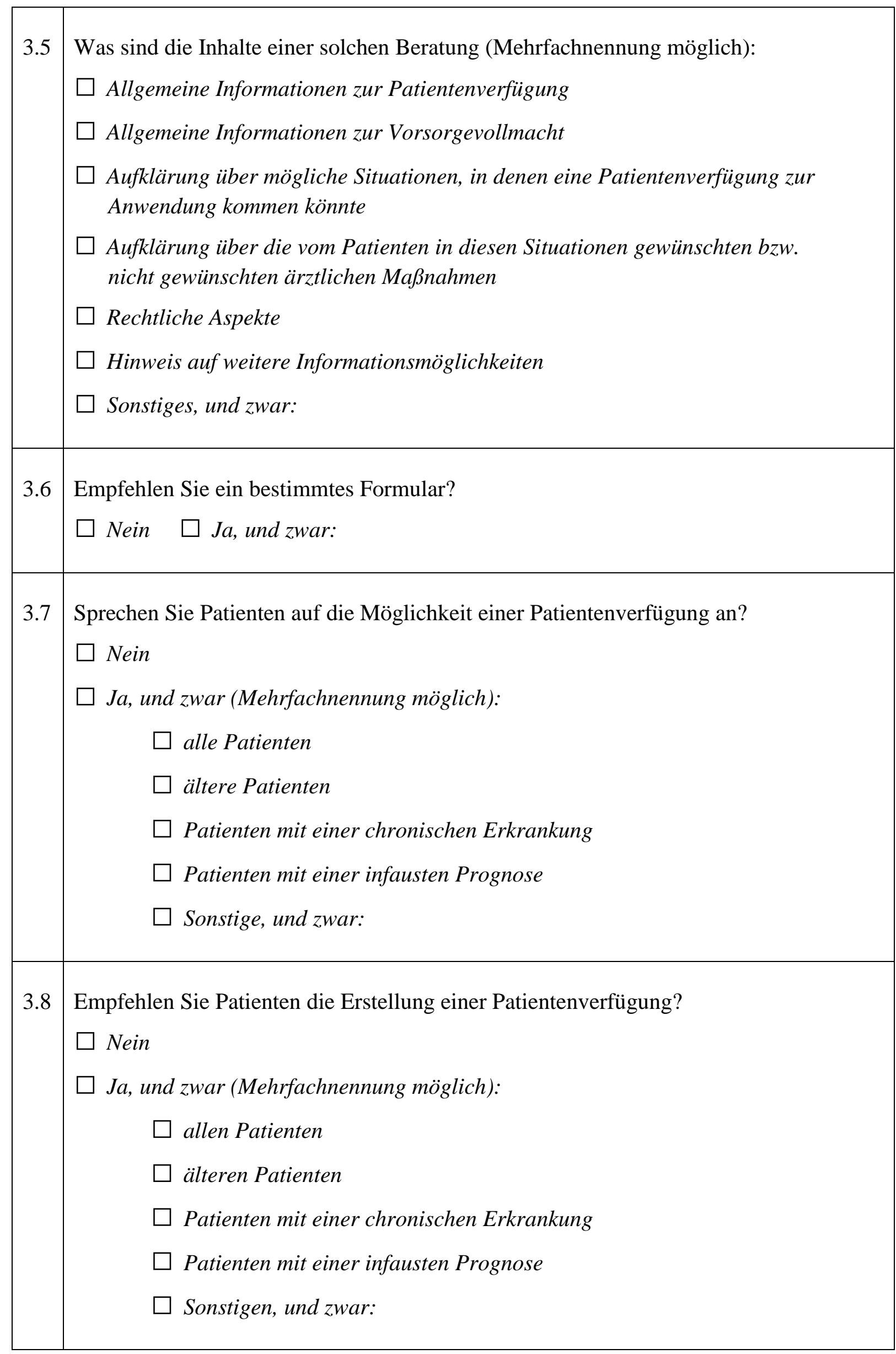


4. Bedeutung der ärztlichen Beratung (Zutreffendes bitte ankreuzen)

4.1 Ohne ärztliche Beratung kann der Patient die möglichen Behandlungssituationen, auf die er in seiner

Patientenverfügung Bezug nimmt (z.B. Sterbephase, Endstadium einer tödlichen Erkrankung,

Wachkoma, fortgeschrittene Demenz), nicht angemessen verstehen.

Ich stimme voll zu $\square \square \square \square \square$ Ich stimme überhaupt nicht zu

4.2 Ohne ärztliche Beratung kann der Patient den möglichen Nutzen und Schaden der ärztlichen

Maßnahmen, zu denen er sich in seiner Patientenverfügung äußert (z.B. Schmerztherapie, künstliche

Ernährung, Beatmung, Medikamente), nicht angemessen einschätzen.

Ich stimme voll zu $\square \square \square \square \square$ Ich stimme überhaupt nicht zu

4.3 Ohne ärztliche Beratung besteht die Gefahr, dass der Wortlaut einer Patientenverfügung nicht mit

dem tatsächlichen Willen des Patienten übereinstimmt.

Ich stimme voll zu $\square \square \square \square \square \quad$ Ich stimme überhaupt nicht zu

4.4 Ohne ärztliche Beratung ist die Patientenverfügung keine Hilfestellung für den Arzt, der später mit der Verfügung konfrontiert wird.

Ich stimme voll zu $\square \square \square \square \square$ Ich stimme überhaupt nicht zu

4.5 Ärztliche Beratung sollte verbindlich sein.

Ich stimme voll zu $\square \square \square \square \square$ Ich stimme überhaupt nicht zu

\section{Kompetenz zur Beratung (Zutreffendes bitte ankreuzen)}

5.1 Ich fühle mich ausreichend kompetent, um Patienten angemessen beraten zu können?

Ich stimme voll zu $\square \square \square \square \square$ Ich stimme überhaupt nicht zu

5.2 Wünschen Sie sich Fortbildung, um Patienten angemessen beraten zu können?

Ja und zwar zu folgenden Themen:

Nein

Weiß nicht 


\section{Finanzierung von Beratung (Zutreffendes bitte ankreuzen)}

6.1 Wie rechnen Sie Beratungsgespräche zur Patientenverfügung ab? (Mehrfachnennung möglich)

Bislang gar nicht

Über Beratungsziffern der GOÄ (Gebührenordnung Ärzte)

Über Beratungsziffern der GKV (Gesetzliche Krankenversicherung)

Als nicht katalogisierte Individuelle Gesundheitsleistung (IGeL)

Sonstiges, und zwar:

6.2 Ich halte die bestehenden Möglichkeiten zur Abrechnung für Beratungsgespräche zur Patientenverfügung für ausreichend.

Ich stimme voll zu $\square \square \square \square \square$ Ich stimme überhaupt nicht zu

6.3 Ärztliche Beratung zur Patientenverfügung sollte:

Eine vom Patienten selbst zu finanzierende Leistung sein

Eine von der Solidargemeinschaft getragene Leistung sein

Sonstiges, und zwar:

7. Platz für weitere Hinweise, Bemerkungen, Kommentare

\section{Herzlichen Dank für Ihre Mitarbeit!}




\subsection{Fragebogen mit Grundauszählung}

Befragung „Beratung zur Patientenverfügung - eine ärztliche Aufgabe?“

\section{Angaben zur Person (Zutreffendes bitte ankreuzen)}

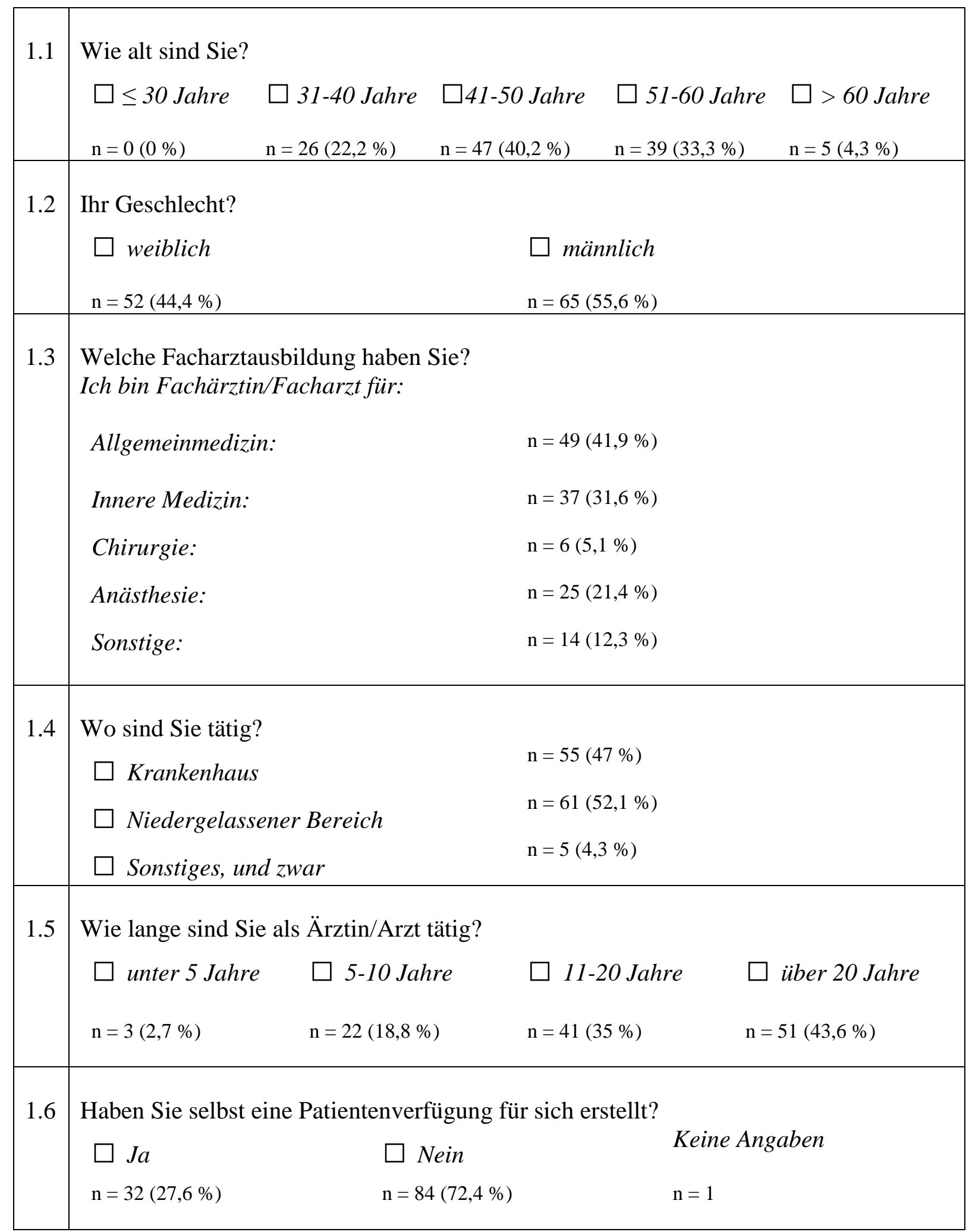




\section{Aktueller Kenntnis- bzw. Informationsstand (Zutreffendes bitte ankreuzen)}

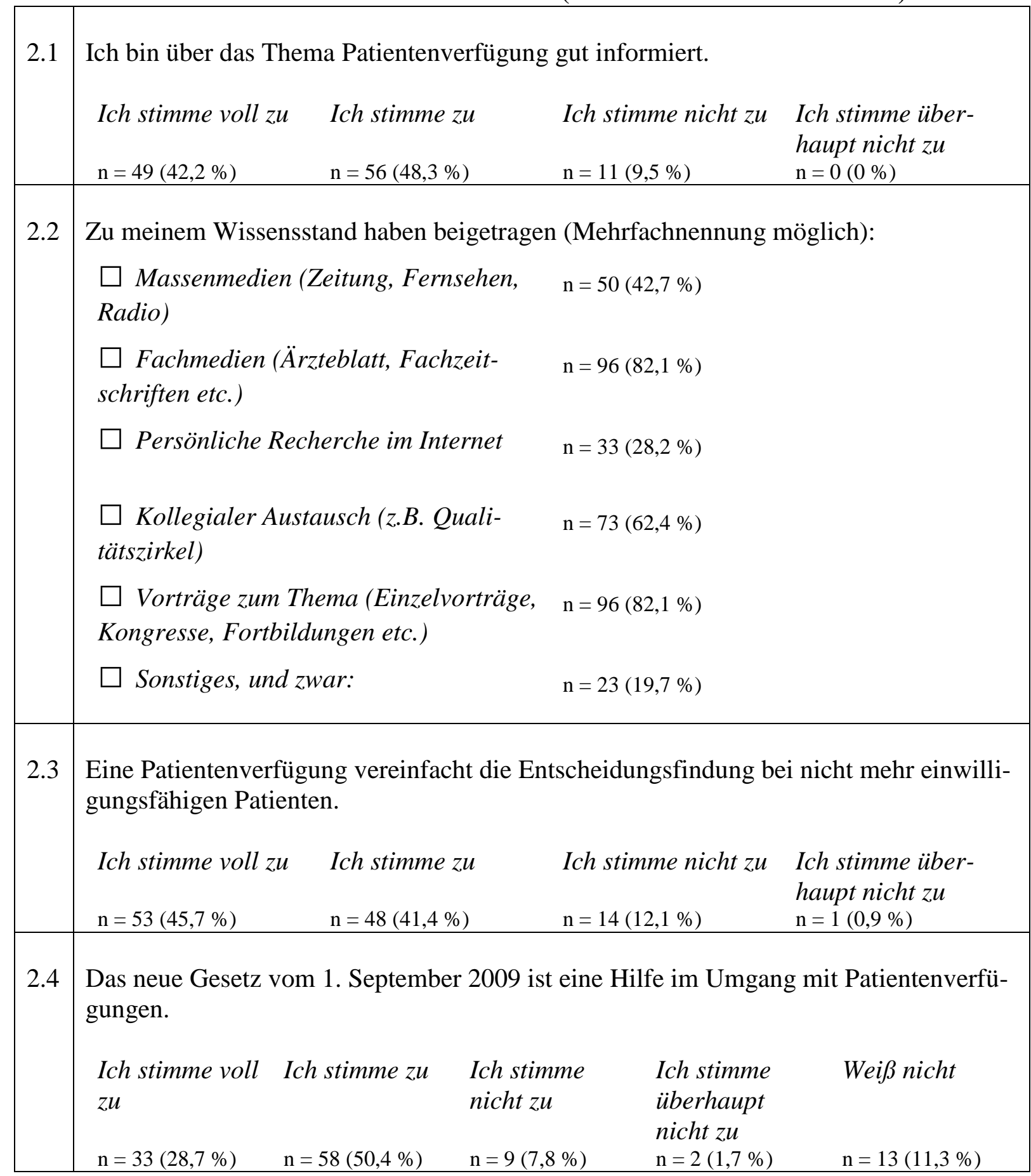

\section{Bisherige Beratungspraxis (Zutreffendes bitte ankreuzen)}

3.1 Wie häufig werden Sie von Patienten auf das Thema Patientenverfügung angesprochen?

$\begin{array}{lllll}\square \text { täglich } & \square \text { wöchent- } & \square \text { monatlich } & \square \text { seltener } & \square \text { noch nie } \\ & \text { lich } & & \\ \mathrm{n}=4(3,4 \%) & \mathrm{n}=36(30,8 \%) & \mathrm{n}=43(36,8 \%) & \mathrm{n}=32(27,4 \%) & \mathrm{n}=2(1,7 \%)\end{array}$




\begin{tabular}{|c|c|c|}
\hline \multirow[t]{4}{*}{3.2} & \multicolumn{2}{|c|}{$\begin{array}{l}\text { Hat die gesetzliche Regelung der Patientenverfügung vom 1. September } 2009 \text { dazu } \\
\text { geführt, dass Sie } \\
\text { nun häufiger von Patienten auf das Thema Patientenverfügung angesprochen werden? }\end{array}$} \\
\hline & Ja, etwas häufiger & $\mathrm{n}=44(38,9 \%)$ \\
\hline & $\begin{array}{l}\text { Nein, die Häufigkeit hat sich nicht merk- } \\
\text { lich verändert }\end{array}$ & $\mathrm{n}=69(61,1 \%)$ \\
\hline & $\begin{array}{l}\text { Nein, die Häufigkeit hat sich eher verrin- } \\
\text { gert }\end{array}$ & $\mathrm{n}=0(0 \%)$ \\
\hline 3.3 & Beraten Sie Patienten zum Thema Patienter & verfügung? \\
\hline & $\begin{array}{l}\text { Nein (weiter mit Frage } 3.7) \\
\mathrm{n}=31(26,7 \%)\end{array}$ & $\begin{array}{l}J a \\
\mathrm{n}=85(73,3 \%)\end{array}$ \\
\hline 3.4 & $\begin{array}{l}\text { Wie lange dauert eine solche Beratung im I } \\
\text { Durchschnittlich ca._________Minuten. (B }\end{array}$ & $\begin{array}{l}\text { urchschnitt? } \\
\text { itte eintragen) }\end{array}$ \\
\hline 3.5 & $\begin{array}{l}\text { Was sind die Inhalte einer solchen Beratun } \\
\text { Allgemeine Informationen zur Patienten- } \\
\text { verfügung }\end{array}$ & $\begin{array}{l}\text { (Mehrfachnennung möglich): } \\
\mathrm{n}=75(64,1 \%)\end{array}$ \\
\hline & $\begin{array}{l}\text { Allgemeine Informationen zur Vorsorge- } \\
\text { vollmacht }\end{array}$ & $\mathrm{n}=73(62,4 \%)$ \\
\hline & $\begin{array}{l}\text { Aufklärung über mögliche Situationen, in } \\
\text { denen eine Patientenverfügung zur An- } \\
\text { wendung kommen könnte }\end{array}$ & $\mathrm{n}=75(64,1 \%)$ \\
\hline & $\begin{array}{l}\text { Aufklärung über die vom Patienten in } \\
\text { diesen Situationen gewünschten bzw. } \\
\text { nicht gewünschten ärztlichen Maßnah- } \\
\text { men }\end{array}$ & $\mathrm{n}=77(65,8 \%)$ \\
\hline & Rechtliche Aspekte & $\mathrm{n}=40(34,2 \%)$ \\
\hline & $\begin{array}{l}\text { Hinweis auf weitere Informationsmög- } \\
\text { lichkeiten }\end{array}$ & $\mathrm{n}=53(45,3 \%)$ \\
\hline & Sonstiges, und zwar: & $\mathrm{n}=11(9,4 \%)$ \\
\hline 3.6 & $\begin{array}{l}\text { Empfehlen Sie ein bestimmtes Formular? } \\
\text { Nein } \\
\mathrm{n}=54(63,5 \%)\end{array}$ & $\begin{array}{l}\text { Ja, und zwar: } \\
\mathrm{n}=29(34,1 \%)\end{array}$ \\
\hline
\end{tabular}




\begin{tabular}{|c|c|c|c|}
\hline \multirow[t]{9}{*}{3.7} & \multicolumn{3}{|c|}{ Sprechen Sie Patienten auf die Möglichkeit einer Patientenverfügung an? } \\
\hline & \multirow{8}{*}{$\begin{array}{l}\text { Keine Angaben } \\
\text { Nein } \\
\text { Ja, und zwar (Mehrfach- } \\
\text { nennung möglich): }\end{array}$} & \multicolumn{2}{|l|}{$\mathrm{n}=3(2,6 \%)$} \\
\hline & & \multicolumn{2}{|l|}{$\mathrm{n}=28(23,9 \%)$} \\
\hline & & \multicolumn{2}{|l|}{$\mathrm{n}=86(73,5 \%)$} \\
\hline & & \multirow{2}{*}{$\begin{array}{l}\text { alle Patienten } \\
\text { ältere Patienten }\end{array}$} & $\mathrm{n}=13(15,1 \%)$ \\
\hline & & & $\mathrm{n}=43(50 \%)$ \\
\hline & & \multirow{2}{*}{$\begin{array}{l}\text { Patienten mit einer chro- } \\
\text { nischen Erkrankung } \\
\text { Patienten mit einer infaus- } \\
\text { ten Prognose }\end{array}$} & $\mathrm{n}=56(65,1 \%)$ \\
\hline & & & $\mathrm{n}=73(84,9 \%)$ \\
\hline & & Sonstige, und zwar: & $\mathrm{n}=16(18,6 \%)$ \\
\hline \multirow[t]{9}{*}{3.8} & \multicolumn{3}{|c|}{ Empfehlen Sie Patienten die Erstellung einer Patientenverfügung? } \\
\hline & Keine Angaben & $\mathrm{n}=2(1,7 \%)$ & \\
\hline & Nein & $\mathrm{n}=10(8,6 \%)$ & \\
\hline & Ja, und zwar (Mehrfach- & $\mathrm{n}=105(89,7 \%)$ & \\
\hline & & alle Patienten & $\mathrm{n}=46(43,8 \%)$ \\
\hline & & ältere Patienten & $\mathrm{n}=75(71,4 \%)$ \\
\hline & & $\begin{array}{l}\text { Patienten mit einer chro- } \\
\text { nischen Erkrankung }\end{array}$ & $\mathrm{n}=77(73,3 \%)$ \\
\hline & & $\begin{array}{l}\text { Patienten mit einer infaus- } \\
\text { ten Prognose }\end{array}$ & $\mathrm{n}=89(84,8 \%)$ \\
\hline & & Sonstige, und zwar: & $\mathrm{n}=19(18,1 \%)$ \\
\hline
\end{tabular}

\section{Bedeutung der ärztlichen Beratung (Zutreffendes bitte ankreuzen)}

4.1 Ohne ärztliche Beratung kann der Patient die möglichen Behandlungssituationen, auf die er in seiner Patientenverfügung Bezug nimmt (z.B. Sterbephase, Endstadium einer tödlichen Erkrankung, Wachkoma, fortgeschrittene Demenz), nicht angemessen verstehen.

\begin{tabular}{llll} 
Ich stimme voll zu & Ich stimme $z u$ & Ich stimme nicht zu & $\begin{array}{l}\text { Ich stimme über- } \\
\text { haupt nicht } z u \\
\mathrm{n}=2(1,7 \%)\end{array}$ \\
\hline $\mathrm{n}=68(58,1 \%)$ & $\mathrm{n}=28(23,9 \%)$ & $\mathrm{n}=19(16,2 \%)$ &
\end{tabular}

4.2 Ohne ärztliche Beratung kann der Patient den möglichen Nutzen und Schaden der ärztlichen Maßnahmen, zu denen er sich in seiner Patientenverfügung äußert (z.B.

Schmerztherapie, künstliche Ernährung, Beatmung, Medikamente), nicht angemessen einschätzen.

$\begin{array}{llll}\text { Ich stimme voll } z u & \text { Ich stimme } z u & \text { Ich stimme nicht } z u & \begin{array}{l}\text { Ich stimme über- } \\ \text { haupt nicht } z u \\ \mathrm{n}=2(1,7 \%)\end{array} \\ \mathrm{n}=67(57,3 \%) & \mathrm{n}=32(27,4 \%) & \mathrm{n}=16(13,7 \%) & \end{array}$


4.3 Ohne ärztliche Beratung besteht die Gefahr, dass der Wortlaut einer Patientenverfügung nicht mit dem tatsächlichen Willen des Patienten übereinstimmt.

$\begin{array}{llll}\text { Ich stimme voll zu } & \text { Ich stimme } z u & \text { Ich stimme nicht zu } & \begin{array}{l}\text { Ich stimme über- } \\ \text { haupt nicht } z u\end{array} \\ \mathrm{n}=52(44,4 \%) & \mathrm{n}=40(34,2 \%) & \mathrm{n}=20(17,1 \%) & \mathrm{n}=5(4,3 \%)\end{array}$

4.4 Ohne ärztliche Beratung ist die Patientenverfügung keine Hilfestellung für den Arzt, der später mit der Verfügung konfrontiert wird.

\begin{tabular}{|c|c|c|c|}
\hline $\begin{array}{l}\text { Ich stimme voll zu } \\
\mathrm{n}=28(24,1 \%)\end{array}$ & $\begin{array}{l}\text { Ich stimme zu } \\
\mathrm{n}=32(27,6 \%)\end{array}$ & $\begin{array}{l}\text { Ich stimme nicht zu } \\
\mathrm{n}=38(32,8 \%)\end{array}$ & $\begin{array}{l}\text { Ich stimme über- } \\
\text { haupt nicht zu } \\
\mathrm{n}=18(15,5 \%)\end{array}$ \\
\hline & verhindlis & & \\
\hline $\begin{array}{l}\text { Ich stimme voll zu } \\
\mathrm{n}=36(31,3 \%)\end{array}$ & $\begin{array}{l}\text { Ich stimme } z u \\
\mathrm{n}=33(28,7 \%)\end{array}$ & $\begin{array}{l}\text { Ich stimme nicht zu } \\
\mathrm{n}=24(20,9 \%)\end{array}$ & $\begin{array}{l}\text { Ich stimme über- } \\
\text { haupt nicht zu } \\
\mathrm{n}=22(19,1 \%)\end{array}$ \\
\hline
\end{tabular}

\section{Kompetenz zur Beratung (Zutreffendes bitte ankreuzen)}

5.1 Ich fühle mich ausreichend kompetent, um Patienten angemessen beraten zu können?

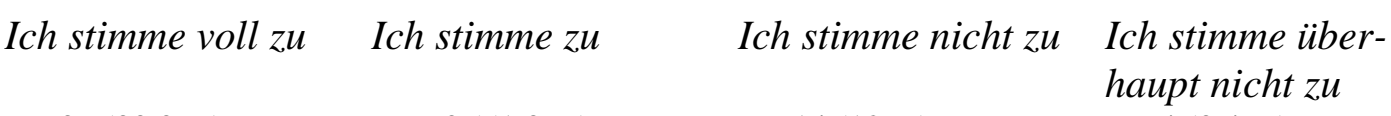

$\mathrm{n}=39(33,3 \%) \quad \mathrm{n}=60(51,3 \%) \quad \mathrm{n}=14(12 \%) \quad \mathrm{n}=4(3,4 \%)$

5.2 Wünschen Sie sich Fortbildung, um Patienten angemessen beraten zu können?

Ja und zwar zu folgenden Themen: $\quad \mathrm{n}=51(44,4 \%)$

Nein

$\mathrm{n}=40(34,8 \%)$

Weiß nicht

$\mathrm{n}=24(20,9 \%)$ 


\section{Finanzierung von Beratung (Zutreffendes bitte ankreuzen)}

6.1 Wie rechnen Sie Beratungsgespräche zur Patientenverfügung ab? (Mehrfachnennung möglich)

Bislang gar nicht

Über Beratungsziffern der GÖ̈ (Gebührenordnung Ärzte)

Über Beratungsziffern der GKV (Gesetzliche Krankenversicherung)

Als nicht katalogisierte Individuelle Gesundheitsleistung (IGeL)

Sonstiges, und zwar:

6.2

Ich halte die bestehenden Möglichkeit
Patientenverfügung für ausreichend.

Ich stimme voll zu Ich stimme zu

$\mathrm{n}=7(8 \%)$

$\mathrm{n}=6(6,8 \%)$

$\mathrm{n}=4(3,4 \%)$

$\mathrm{n}=6(5,1 \%)$

Keine Angaben: $\mathrm{n}=29(33 \%)$

6.3 Ärztliche Beratung zur Patientenverfügung sollte:

Eine vom Patienten selbst zu finanzie- $\quad \mathrm{n}=40(35,7 \%)$

rende Leistung sein

Eine von der Solidargemeinschaft getra- $\quad \mathrm{n}=63(56,3 \%)$

gene Leistung sein

Sonstiges, und zwar:

$\mathrm{n}=9(8 \%)$

Keine Angaben: $\mathrm{n}=5$ 
7. Platz für weitere Hinweise, Bemerkungen, Kommentare

- Das Thema Vorsorgevollmacht sollte ebenfalls breiter diskutiert werden

- Spezielle Ziffer sollte anzusetzen sein zu Lasten der GKV

- Mehr öffentliche Aufklärungsarbeit besonders über die Medien erforderlich

- PV sollte von der Solidargemeinschaft getragen werden, denn es ist eine gesetzliche Regelung, also auch soziale Verpflichtung und Gemeinschaftsleistung

- Patientenverfügung immer mit Vorsorgevollmacht koppeln!

- Ohne weitere Vergütung keine weitere Aufgabe im Bereich GOÄ

- Im Fragebogen nicht genau formuliert, ob es nur um die Erstellung geht oder auch um die Umsetzung als Anästhesisten im OP/Intensiv/Notarzt habe ich nur mit Umsetzung zu tun. Ich vermisse im Krankenhaus ständig die rechtzeitige Klärung des Procedere bei akuter Zustandsverschlechterung bei schwerkranken Patienten, keiner hat Zeit

- 5.1 differenziert nicht zwischen allgemeiner Beratung und fachlicher Beobachtung; 6.3 unleserlich

- Bessere Aufklärung vor großen (auch tumor-) chirurgischen Eingriffen sollte auch den Hinweis auf das Erstellen einer Patientenverfügung einschließlich Vorsorgevollmacht beinhalten, sowie die möglichen Komplikationen des Eingriffes

- Im Verlauf einer Intensivbehandlung ergeben sich bezüglich einer Patientenverfügung oft folgende Probleme: -der Patient und seine Angehörigen haben keine Kenntnis

- Auseinandersetzung mit dem Thema und Info über Entscheidung an Angehörige oder schriftliche sollte Pflicht sein!

\section{Herzlichen Dank für Ihre Mitarbeit!}




\subsection{Literaturverzeichnis}

AG „Patientenautonomie am Lebensende“ (2004): Ethische, rechtliche und medizinische Aspekte zur Bewertung von Patientenverfügungen. Bericht der Arbeitsgruppe „Patientenautonomie am Lebensende" vom 10. Juni 2004 http://www.bmj.de/SharedDocs/Downloads/DE/pdfs/Patientenautonomie_am_ Lebensende.pdf?__blob=publicationFile (letzter Zugriff am 13.11.2012)

BÄK (2007): Empfehlungen der Bundesärztekammer und der Zentralen Ethikkommission bei der Bundesärztekammer zum Umgang mit Vorsorgevollmacht und Patientenverfügung in der ärztlichen Praxis. Dtsch Ärztebl 104 (13), A-891-896.

BÄK (2010): Empfehlungen der Bundesärztekammer und der Zentralen Ethikkommission bei der Bundesärztekammer zum Umgang mit Vorsorgevollmacht und Patientenverfügung in der ärztlichen Praxis. Dtsch Ärztebl 107 (18), A 877-882 http://www.bundesaerztekammer.de/downloads/Patientenverfuegung_und_Vollma cht_Empfehlungen_BAeK-ZEKO_DAe1.pdf (letzter Zugriff 13.11.2012)

BÄK (2011): Grundsätze der Bundesärztekammer zur ärztlichen Sterbebegleitung. Dtsch Ärztebl $\underline{108}$ (7), A 347, 18. Februar 2011 http://www.bundesaerztekammer.de/downloads/Sterbebegleitung_17022011.pdf (letzter Zugriff 13.11.2012)

Bayerisches Staatsministerium der Justiz und für Verbraucherschutz - Referat für Öffentlichkeitsarbeit - Prielmayerstraße 7, 80335 München, Stand: Oktober 2011, 12. Auflage http://www.verwaltung.bayern.de/egov-portlets/xview/Anlage/1928142/ VorsorgefuerUnfall,KrankheitundAlter.pdf (letzter Zugriff am 18.11.2012)

Becker M, Jaspers B, Clemens K, Klaschik E, Radbruch L, Voltz R, Nauck F (2009): How to explore what people really want to say with an advanced directive (Poster abstract). http://www.eapcnet.org/vienna2009/download/Vienna_Abstracts_2009.pdf (letzter Zugriff am 13.11.2012)

BGB §1896 Abs. 2, in der Fassung der Bekanntmachung vom Januar 2002 (Bundesgesetzblatt I, 42, 2909;2003 I, 738), zuletzt geändert durch Art. 2 G v. 15.3.2012 || 178. http://www.gesetze-im-internet.de/bgb/_1896.html (letzter Zugriff 04.12.2012) 
BGB §1897 Abs. 4, in der Fassung der Bekanntmachung vom Januar 2002 (Bundesgesetzblatt I, 42, 2909;2003 I, 738), zuletzt geändert durch Art. 2 G v. 15.3.2012 II 178. http://www.gesetze-im-internet.de/bgb/_1897.html (letzter Zugriff 04.12.2012)

BGB §1901a Abs. 1, in der Fassung der Bekanntmachung vom Januar 2002 (Bundesgesetzblatt I, 42, 2909;2003 I, 738), zuletzt geändert durch Art. 2 G v. 15.3.2012 II 178. http://www.gesetze-im-internet.de/bgb/_1901a.html (letzter Zugriff 04.12.2012)

BGB § 1901c, in der Fassung der Bekanntmachung vom Januar 2002 (Bundesgesetzblatt I, 42, 2909;2003 I, 738), zuletzt geändert durch Art. 2 G v. 15.3.2012 II 178. http://www.gesetze-im-internet.de/bgb/_1901c.html (letzter Zugriff 04.12.2012)

BGB §1904 Abs. 5, in der Fassung der Bekanntmachung vom Januar 2002 (Bundesgesetzblatt I, 42, 2909;2003 I, 738), zuletzt geändert durch Art. 2 G v. 15.3.2012 II 178. http://www.gesetze-im-internet.de/bgb/_1904.html (letzter Zugriff 04.12.2012)

BGB §1906, in der Fassung der Bekanntmachung vom Januar 2002 (Bundesgesetzblatt I, 42, 2909;2003 I, 738), zuletzt geändert durch Art. 2 G v. 15.3.2012 II 178. http://www.gesetze-im-internet.de/bgb/_1906.html (letzter Zugriff 04.12.2012)

BGH (2003): Beschluss vom 17.03.2003 - XII ZB 2/03 (Schleswig). Neue Juristische Wochenschrift $\underline{56}(22), 1588-1594$.

Bosbach W, Röspel R, Göring-Eckardt K, Terpe H, Winkler J, Fricke O, Hasselfeldt G, Thierse W, Kauder V, Künast R (2008): Entwurf eines Gesetzes zur Verankerung der Patienten-verfügung im Betreuungsrecht (Patientenverfügungsgesetz - PatVerfG). BT Drucks. $16 / 11360$.

http://www.aem-online.de/d2o4w6n8l103a5d7f9i2/4e6s/90_ge_pv_bosbach.pdf (letzter Zugriff: 13.11.2012)

Bostridge M: Florence Nightingale. Penguin Books, London 2009

3. BtÄndG (= Drittes Gesetz zur Änderung des Betreuungsrechts) vom 29. Juli 2009 (Bundesgesetzblatt 2009 I, 2286)

Deutsche Hospizstiftung (2005): Wie denken die Deutschen über Patientenverfügungen? http://www.hospize.de/docs/stellungnahmen/32.pdf (letzter Zugriff 13.11.2012) 
Deveugele M, Derese A, van den Brink-Muinen A, Bensing J, De Maeseneer J. (2002): Consultation length in general practice: cross sectional study in six European countries. BMJ $\underline{325}$ [7362], Seite 472.

Duden: Band 1. Die deutsche Rechtschreibung, Dudenverlag, Mannheim 2000, 22. Auflage S. 398

Enquete-Kommission Ethik und Recht der modernen Medizin (2004): Zwischenbericht Patientenverfügungen. http://dipbt.bundestag.de/dip21/btd/15/037/1503700.pdf (letzter Zugriff: 13.11.2012)

Faden R, Beauchamp T: A history and theory of informed consent. Oxford University Press, Oxford 1986

Genfer Deklaration (1948): Verabschiedet von der 2. Generalversammlung des Weltärztebundes, Genf, Schweiz, September 1948, zuletzt revidiert von der 46. Generalversammlung des Weltärztebundes Stockholm, Schweden, September 1994

GG Art. 1, Abs. 1, in der im Bundesgesetzblatt Teil III, Gliederungsnummer 100-I, veröffentlichen bereinigten Fassung, zuletzt geändert durch Art. 1 ÄndG vom 11. Juli 2012 (BGBI. I S. 1478). http://www.gesetze-im-internet.de/gg/art_1.html (letzter Zugriff 04.12.2012)

GG Art. 2 Abs. 1, in der im Bundesgesetzblatt Teil III, Gliederungsnummer 100-I, veröffentlichen bereinigten Fassung, zuletzt geändert durch Art. 1 ÄndG vom 11. Juli 2012 (BGBI. I S. 1478). http://www.gesetze-im-internet.de/gg/art_2.html (letzter Zugriff 04.12.2012)

GKV-WSG (2007): Entwurf eines Gesetzes zur Stärkung des Wettbewerbs in der gesetzlichen Krankenversicherung (GKV-Wettbewerbsstärkungsgesetz - GKV-WSG). BT Drucks. 16/3100. http://dipbt.bundestag.de/dip21/btd/16/031/1603100.pdf (letzter Zugriff: 04.12.2012)

Hillgruber C (2006): Die Würde des Menschen am Ende seines Lebens Verfassungsrechtliche Anmerkungen. Z Lebensrecht $\underline{15}$ (3), 70-81.

Hufen F: Geltung und Reichweite von Patientenverfügungen. Nomos, Baden-Baden 2009

Jährig, C, Koch U: Die Arzt-Patient-Interaktion in der internistischen Visite eines Akutkrankenhauses - Eine empirische Untersuchung. In: Köhle, K., Raspe, H.-H. (Hrsg.) Das 
Gespräch während der ärztlichen Visite, Urban und Schwarzenberg München, Wien, Baltimore 1982, S. 36-57

Jaspers B, Becker M, King C, Radbruch L, Voltz R, Nauck F: „Ich will nicht so sterben wie mein Vater!“ Eine qualitative Untersuchung zum Einfluss von Motivationen auf die Konzeption einer Patientenverfügung. Z Palliativmed $\underline{11}$ (2010) S. 218 - 226

Kämpfer U: Das Recht auf den eigenen Tod - Sterbehilfe im deutschen Verfassungs-recht. In:

Die Freiheit zu sterben. Heinrich Böll Stiftung (Hg) Berlin 2007, S.79-100

http://www.boell.de/downloads/bildungkultur/Buch_Die_Freiheit_zu_sterben.pdf (letzter Zugriff 13.11.2012)

Lichtenthaeler C: Der Eid des Hippokrates. Deutscher Ärzte-Verlag GmbH, Köln 1984

Oorschot van B, Simon A (2006): Importance of the advance directive and the beginning of the dying process from the point of view of German doctors and judges dealing with guardianship matters: results of an empirical survey. J Med Ethics $\underline{32}$ (11), S. 623-626.

Sahm S: Sterbebegleitung und Patientenverfügung. Ärztliches Handeln an den Grenzen von Ethik und Recht. Campus, Frankfurt am Main 2006

Schäfer D: Patientenverfügungen: „Krank - aber entscheidungsfähig.“ Jacobs, Lage 2001

Simon A, Lipp V, Tietze A, Nickel N, van Oorschot B (2004): Einstellung deutscher Vormundschaftsrichterinnen und -richter zu medizinischen Entscheidungen und Maßnahmen am Lebensende. Medizinrecht 22 (6), S. 303-307.

SGB V §73b, Gesetzliche Krankenversicherung, (Artikel 1 des Gesetzes vom 20. Dezember 1988, BGBI. I S. 2477), das zuletzt durch Artikel 3 des Gesetzes vom 23. Oktober 2012 (BGBI. I S. 2246) geändert worden ist. http://www.gesetze-iminternet.de/sgb_5/_73b.html (letzter Zugriff 04.12.2012)

St GB $\S 223$, in der Fassung der Bekanntmachung vom 13. November 1998 (Bundesgesetzblatt I, 3322), zuletzt geändert durch Art. 1 G vom 25. Juni 2012 (BGBI. I S. 1374). http://www.gesetze-im-internet.de/stgb/_223.html (letzter Zugriff 04.12.2012)

Stünker J, Kauch M, Jochimsen L, Montag J, Addicks K, Andreae K, Arndt-Brauer I, Arnold R, Bätzing S, Bahr D (2008): Entwurf eines Dritten Gesetzes zur Änderung des Betreuungs-rechts. BT-Drucks. 16/8442. 
http://www.aem-online.de/d2o4w6n8l1o3a5d7f9i2|4e6s/

89_ge_pv_stuenker_maerz08.pdf (letzter Zugriff 13.11.2012)

Taupitz J: Die Debatte um ein Patientenverfügungsgesetz. In: Junginger T, Pereczky A, Vahl C

(Hg): Grenzsituationen in der Intensivmedizin. Springer, Heidelberg 2008, S. 113-123.

Verrel, T: Patientenautonomie und Strafrecht bei der Sterbebegleitung. Gutachten C zum 66. Deutschen Juristentag. C.H. Beck, München 2006

Weber M, Stiehl M, Rittner C, Reiter J (2001): Ethische Entscheidungen am Ende des Lebens. Sorgsames Abwägen der jeweiligen Situation. Ergebnis einer Ärztebefragung in Rheinland-Pfalz. Dtsch Ärztebl $\underline{98}$ (48), A-3184-3188.

Wissenschaftliches Institut der Privaten Krankenversicherungen (2006): „Alter und steigende Lebenserwartung - Eine Analyse der Auswirkungen auf die Gesundheitsausgaben, Dr. Frank Niehaus http://www.wippkv.de/uploads/tx_nppresscenter/Alter_und_steigende_Lebenserwa rtung.pdf (letzter Zugriff 13.11.2012)

Zöller W, Faust H, Däubler-Gmelin H, Knoche M, Aydin H, Bauer W, Binninger C, BullingSchröter E, Dehm D, Dreibuset W (2008): Entwurf eines Gesetzes zur Klarstellung der Verbindlichkeit von Patientenverfügungen (Patientenverfügungsverbindlichkeitsgesetz-PvvG). BT-Drucks. 16/11493.

http://www.aem-online.de/d2o4w6n8|103a5d7f9i2/4e6s/104_ge_pv_zoeller.pdf (letzter Zugriff 13.11.2012) 


\section{Danksagung}

Mein besonderer Dank gilt meinem Doktorvater Herrn PD Dr. phil. A. Simon für ausdauernde Geduld und Beratung.

Sehr bedanken möchte ich mich auch bei Herrn Dr. rer. nat. K. Jung der Abteilung Medizinische Statistik und seinen Kollegen, die mich bei der statistischen Auswertung der Ergebnisse unterstützt und mich immer freundlich und gut beraten haben. 


\section{Lebenslauf}

Am 11.09.1985 wurde ich als Tochter einer Lehrerin und eines Chirurgen in Bielefeld geboren. Ich wuchs in Brake, einem Vorort Bielefelds, auf und besuchte dort auch die Grundschule. Ab der fünften Klasse besuchte ich das Ceciliengymnasium in Bielefeld Mitte, wo ich 2005 mit dem Abitur abschloss. Es folgte ein siebenmonatiger Travel and Work Aufenthalt in Australien zur Verbesserung meiner Englischkenntnisse und Selbstständigkeit. Zum Sommersemester 2006 begann ich das Studium der Humanmedizin an der GeorgAugust-Universität in Göttingen. Den vorklinischen Studienteil schloss ich im Frühjahr 2008 mit dem Ersten Abschnitt der ärztlichen Prüfung ab. Im klinischen Abschnitt famulierte ich in der Kardiologie, Intensivmedizin, Gynäkologie und Allgemeinmedizin. Mein praktisches Jahr absolvierte ich zunächst in der Abteilung für Innere Medizin in der Ammerland-Klinik in Westerstede. Es folgte ein zweimonatiger Aufenthalt in Nepal im Rahmen des Chirurgietertials. Für die zweite Hälfte dieses Tertials kehrte ich zur Ammerland-Klinik zurück. Mein Wunschtertial absolvierte ich schließlich in einer allgemeinmedizinischen Praxis in Göttingen. Nach Bestehen des zweiten Abschnitts der ärztlichen Prüfung und Erhalten der Approbation bin ich seit August 2012 in der Abteilung für Allgemeinchirurgie in der Ammerland-Klinik als Assistenzärztin angestellt.

Seit 2009 promoviere ich unter Anleitung von Herrn PD Dr. A. Simon in der Abteilung Ethik und Geschichte der Medizin der Universitätsmedizin Göttingen. 